Florida International University FIU Digital Commons

6-28-2017

\title{
Studies of Ne-Lysine Acetylation Modification on Escherichia coli Topoisomerase I
}

Qingxuan Zhou

Florida International University, qzhou004@fiu.edu

DOI: $10.25148 /$ etd.FIDC001972

Follow this and additional works at: https://digitalcommons.fiu.edu/etd

Part of the Biochemistry Commons

\section{Recommended Citation}

Zhou, Qingxuan, "Studies of Ne-Lysine Acetylation Modification on Escherichia coli Topoisomerase I" (2017). FIU Electronic Theses and Dissertations. 3361.

https://digitalcommons.fiu.edu/etd/3361

This work is brought to you for free and open access by the University Graduate School at FIU Digital Commons. It has been accepted for inclusion in FIU Electronic Theses and Dissertations by an authorized administrator of FIU Digital Commons. For more information, please contact dcc@fiu.edu. 


\section{FLORIDA INTERNATIONAL UNIVERSITY}

Miami, Florida

\section{STUDIES OF N ${ }^{\varepsilon}$-LYSINE ACETYLATION MODIFICATION ON ESCHERICHIA COLI TOPOISOMERASE I}

A dissertation submitted in partial fulfillment of

the requirements for the degree of

DOCTOR OF PHILOSOPHY

in

BIOCHEMISTRY

by

Qingxuan Zhou 
To: Dean Michael R. Heithaus

College of Arts, Sciences and Education

This dissertation, written by Qingxuan Zhou, and entitled Studies of Ne-Lysine Acetylation Modification on Escherichia coli Topoisomerase I, having been approved in respect to style and intellectual content, is referred to you for judgment.

We have read this dissertation and recommend that it be approved.

$\begin{array}{r}\hline \text { Low W. Kim } \\ \hline \text { Watson J. Lees } \\ \hline \text { Yuan Liu } \\ \hline \text { Xiaotang Wang } \\ \hline \text { Yuk-Ching Tse-Dinh, Major Professor }\end{array}$

Date of Defense: June 28, 2017

The dissertation of Qingxuan Zhou is approved.

Dean Michael R. Heithaus College of Arts, Sciences and Education

Andrés G. Gil Vice President for Research and Economic Development and Dean of the University Graduate School

Florida International University, 2017 
C Copyright 2017 by Qingxuan Zhou

All rights reserved 


\section{DEDICATION}

I dedicate this dissertation to my parents and grandparents for their love, encouragement and support. 


\section{ACKNOWLEDGMENTS}

This dissertation would not have been possible without the help and support I received throughout my Ph.D. study. I would like to express my deepest gratitude to my advisor, Dr. Yuk-Ching Tse-Dinh for her thoughtful guidance and support. From her, I have learnt what it means to be a great mentor and what it takes to become a great scientist. She encouraged and supported every step of my professional growth.

I would like to thank my committee members, Dr. Kim, Dr. Lees, Dr. Liu and Dr. Wang for their advice and support during my $\mathrm{Ph} . \mathrm{D}$. training.

I would like to thank Dr. Thiranavukkarasu Annamalai, and Dr. Gagandeep Narula, who taught me everything at the bench. I would also like to thank my fellow Ph. D. students Nan Cao, Srikanth Banda, Shayna Sandhaus and Pamela Garcia for their help.

Lastly, I would like to thank my amazing friend Xiao Jin, who has been my source of fun and encouragement over many years. I am blessed to have her in my life. 


\section{ABSTRACT OF THE DISSERTATION \\ STUDIES OF ${ }^{\varepsilon}$-LYSINE ACETYLATION MODIFICATION ON ESCHERICHIA COLI TOPOISOMERASE I}

by

Qingxuan Zhou

Florida International University, 2017

Miami, Florida

Professor Yuk-Ching Tse-Dinh, Major professor

Escherichia coli topoisomerase I (TopA), a regulator of global and local DNA supercoiling, is modified by $\mathrm{N}^{\varepsilon}$-Lysine acetylation. The sirtuin protein deacetylase CobB can reverse both enzymatic and non-enzymatic lysine acetylation modifications. Here, we explored the effect of lysine acetylation on E. coli topoisomerase I through analysis of TopA relaxation activity and protein expression in cell extract of wild-type and a $\triangle \operatorname{cobB}$ mutant strains. We showed that the absence of deacetylase CobB in a $\triangle c o b B$ mutant reduced intracellular TopA relaxation activity while elevating TopA expression and topA gene transcripts levels. Acetyl phosphate mediated lysine acetylation decreased the activity of purified TopA in vitro, and the interaction with purified CobB protected TopA from such inactivation. We explored the physiological significance of TopA acetylation on DNA supercoiling by two-dimensional gel analysis and on cell growth rate by growth curve analysis. We found that the absence of CobB increased negative DNA supercoiling. The 
slow growth phenotype of the $\triangle c o b B$ mutant can be partially compensated by overexpression of recombinant TopA. In addition, the specific activity of TopA expressed from His-tagged fusion construct in the chromosome was inversely proportional to the degree of in vivo lysine acetylation during growth transition and growth arrest. Investigation of TopA relaxation mechanism using nuclease footprinting and TopA oxidative crosslinking suggested the potential association of TopA acetylation in catalysis. Mass spectrometry analysis of in vitro acetyl phosphate acetylated TopA identified abundant lysine acetylation sites. Substitution of lysine residues by site-directed mutagenesis was used to model the effect of acetylation on individual lysine residues. Our results showed that substitution of Lys -484 with alanine reduced the relaxation activity, suggesting the reduction of TopA relaxation activity by acetylation was probably in part due to acetylation on Lys-484. These findings demonstrate that E. coli topoisomerase I is modulated by lysine acetylation and the prevention of TopA inactivation from excess lysine acetylation and consequent increase in negative DNA supercoiling is an important physiological function of the sirtuin deacetylase CobB. 
CHAPTER

PAGE

INTRODUCTION

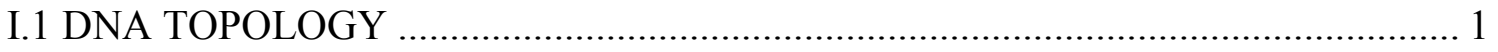

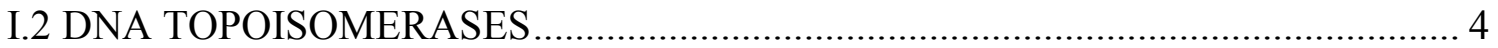

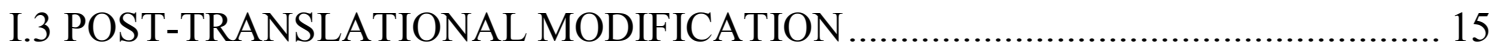

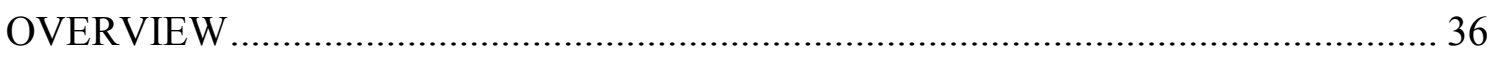

CHAPTER ONE: ACETYLATION REDUCES E. COLI TOPIOSMERASE I CATALYTIC ACTIVITY AND THE SIRTUIN DEACETYLASE COBB

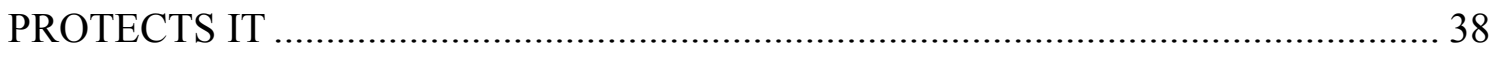

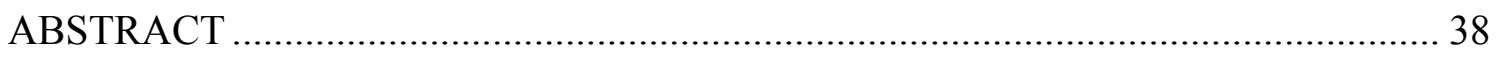

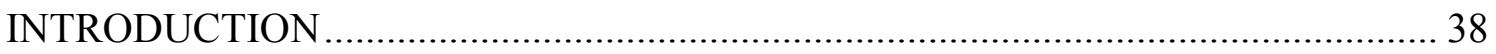

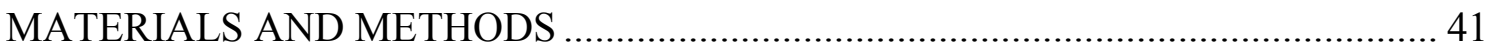

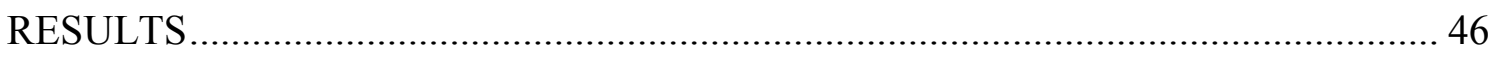

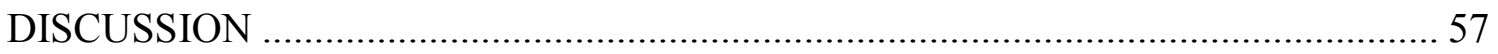

CHAPTER TWO: DEACETYLATION OF TOPOISOMERASE I IS AN IMPORTANT PHYSIOLOGICAL FUNCTION OF E. COLI COBB ........................... 59

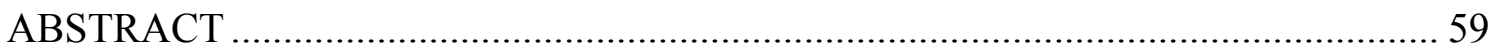

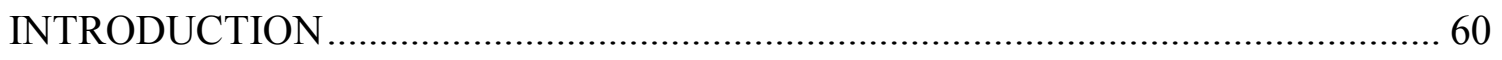

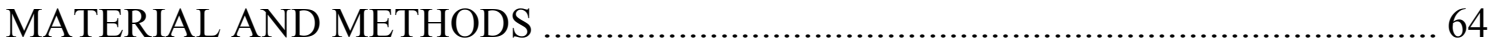

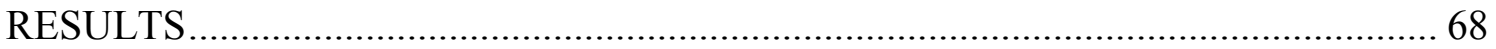

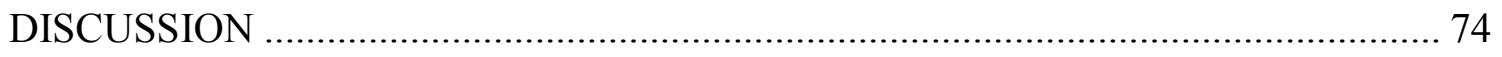

CHAPTER THREE: INVESTIGATION OF MOLECULAR MECHANISM OF $E$. COLI TOPOISOMERASE I ACTIVITY REDUCTION BY ACETYLATION ……..... 77

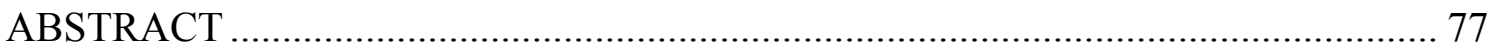

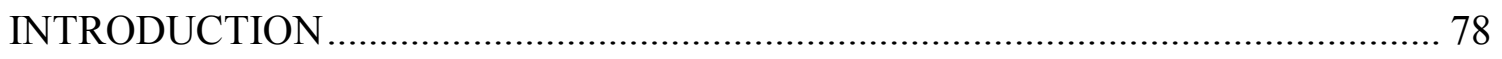

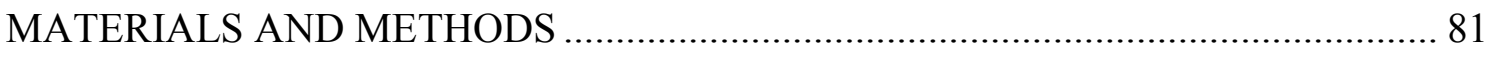

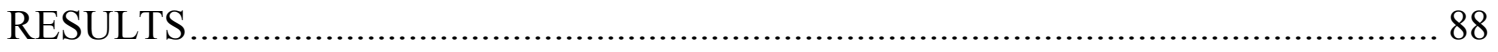

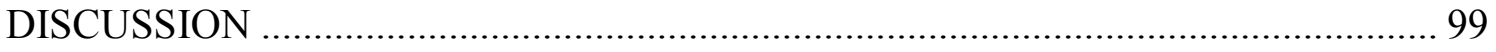

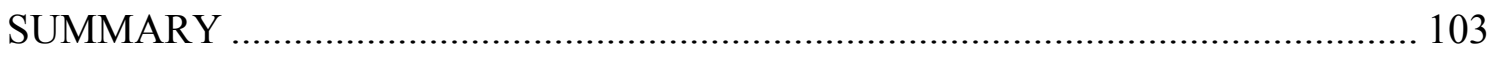

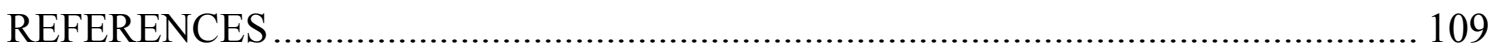

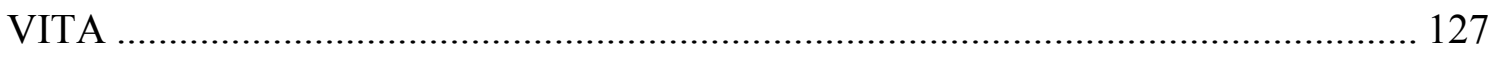




\section{LIST OF TABLES}

TABLES

PAGES

Table 1 The subfamilies and representative members of DNA topoisomerases ............... 5

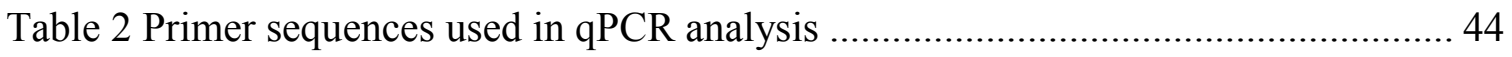

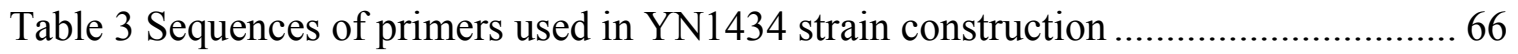

Table 4 Primers used in site-directed mutagenesis ............................................... 85

Table 5 Mass spectrometry identified acetylated lysine sites in acP acetylated TopA.... 96 


\section{LIST OF FIGURES}

FIGURE

PAGE

\section{INTRODUCTION}

Figure 1 Structures of negative and positive DNA supercoilings................................. 2

Figure 2 Topological problems during DNA replication and transcription...................... 3

Figure 3 Catalysis of DNA transient breakage by DNA topoisomerases ........................ 5

Figure 4 Catalytic mechanisms of topoisomerases ............................................... 6

Figure 5 Topoisomerase functions during DNA replication...................................... 8

Figure 6 Topoisomerase functions during DNA transcription ................................. 9

Figure 7 Organization and structure of type IA topoisomerases ................................ 11

Figure 8 Enzyme-bridged strand passage mechanism of type IA topoisomerase ........... 13

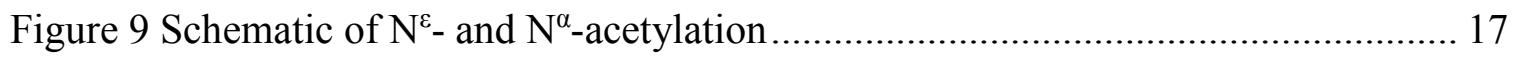

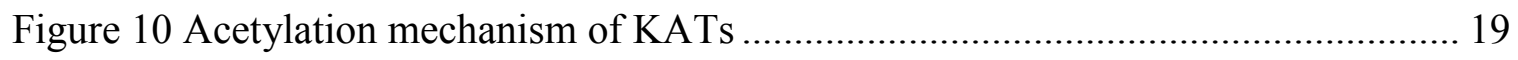

Figure 11 Deacetylation mechanisms of $\mathrm{Zn}^{2+}$ dependent HDACs and NAD ${ }^{+}$dependent

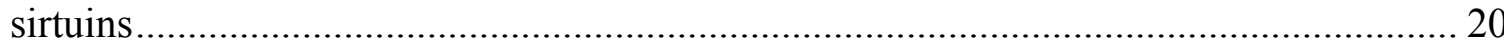

Figure 12 Functional consequences of lysine acetylation........................................ 21 Figure 13 The analytical strategy and method for global profiling of lysine acetylation. 24

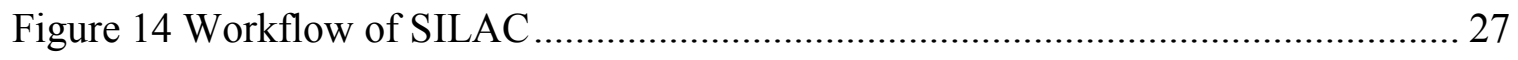

Figure 15 Schematic of the contributions of CoA and acetyl-CoA to cellular metabolism.

Figure 16 Domain structure of Salmonella enterica Pat................................................ 33

Figure 17 The phosphotransacetylase (Pta)-acetate kinase (AckA) pathway.................. 34 


\section{CHAPTER ONE}

Figure 18 Relaxation assay of TopA activity in total soluble cell lysates of BW25113 (wild-type) and JW1106 $(\triangle \operatorname{cobB})$ and their pCobB transformants

Figure 19 Coomassie blue staining of total cellular proteins in cell extract of wild-type BW25113 (wild-type) and $\triangle$ cobB mutant JW1106

Figure 20 TopA expression level in BW25113 (wild-type) and JW1106 ( $\triangle c o b B)$ strains50

Figure 21 TopA transcripts level in BW25113 (wild-type) and JW1106 ( $\triangle$ cobB) strains 51

Figure 22 Acetyl phosphate mediated nonenzymatic lysine acetylation results in decrease in TopA catalytic activity

Figure 23 CobB deacetylation counters the effect of acetyl phosphate mediated lysine

acetylation on TopA relaxation activity.

Figure 24 Direct interaction between TopA and CobB 56

\section{CHAPTER TWO}

Figure 25 The SOS response.

Figure 26 2-Dimensional chloroquine gel analysis comparing DNA supercoiling in BW25113 (wild-type) and JW1106 ( $\triangle c o b B)$ mutant strains

Figure 27 Effect of recombinant TopA overexpression on the slow-growth phenotype

of the $\triangle c o b B$ mutant.

Figure 28 TopA specific activity is inversely proportional to degree of lysine acetylation during growth transition and growth arrest.

\section{CHAPTER THREE}

Figure 29 Positions of lysine acetylation in full length TopA in bound with two single stranded DNA 80

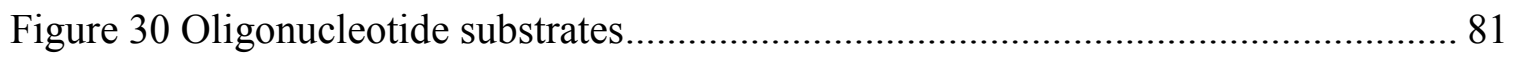

Figure 31 Comprehensive structure of TopA in complex with two ssDNA..................... 88

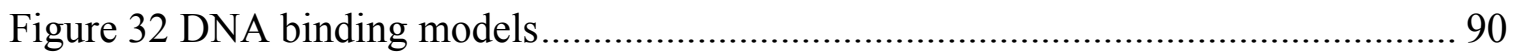


Figure 33 DNase I footprinting with the Bubble substrate

Figure 34 MNase footprinting with the Bubble substrate........................................ 92

Figure 35 Positions of crosslinked lysine residues in TopA ....................................... 94

Figure 36 Positions of of mass spectrometry identified acetylated lysine sites in acP

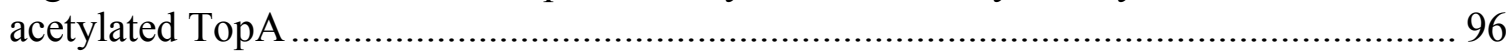

Figure 37 Relaxation assay comparing wild-type TopA with mutant TopA................... 98 


\section{ABBREVIATIONS AND ACRONYMS}

\begin{tabular}{|c|c|}
\hline $\mathrm{AcCoA}$ & Acetyl Coenzyme A \\
\hline $\mathrm{AcP}$ & Acetyl phosphate \\
\hline Acs & Acetyl-CoA synthetase \\
\hline ATP & Adenosine triphosphate \\
\hline BSA & Bovine serum albumin \\
\hline cDNA & Complementary DNA \\
\hline$\Delta$ & Delta, gene deleted \\
\hline DNase I & Deoxyribonuclease I \\
\hline dsDNA & double-stranded DNA \\
\hline DTT & Dithiothreitol \\
\hline E. coli & Escherichia coli \\
\hline EDTA & Ethylenediaminetetraacetic acid \\
\hline EP & Exponential phase \\
\hline GNAT & Gcn5-related N-acetyltransferase \\
\hline $\mathrm{HCl}$ & Hydrogen chloride \\
\hline HEPES & 4-(2-hydroxyethyl)-1-piperazineethanesulfonic acid \\
\hline IPTG & Isopropyl $\beta$-D-1-thiogalactopyranoside \\
\hline KAT & Lysine acetyltransferase \\
\hline $\mathrm{KCl}$ & Potassium chloride \\
\hline $\mathrm{kDa}$ & kilo Dalton \\
\hline KDAC & Lysine deacetylase \\
\hline $\mathrm{KH}_{2} \mathrm{PO}_{4}$ & Potassium phosphate monobasic \\
\hline LB & Lysogeny broth \\
\hline
\end{tabular}




\begin{tabular}{|c|c|}
\hline Lys & Lysine \\
\hline $\mathrm{MgCl}_{2}$ & Magnesium chloride \\
\hline MNase & Micrococcal nuclease \\
\hline MS & Mass spectrometry \\
\hline $\mathrm{Na}_{2} \mathrm{SO}_{3}$ & Sodium sulfite \\
\hline $\mathrm{NaCl}$ & Sodium chloride \\
\hline NAT & N-terminal acetyltransferase \\
\hline NAD & Nicotinamide adenine dinucleotide \\
\hline $\mathrm{NaH}_{2} \mathrm{PO}_{4}$ & Sodium phosphate monobasic \\
\hline NAM & Nicotinamide \\
\hline $\mathrm{NaIO}_{4}$ & Sodium periodate \\
\hline OD & Optical density \\
\hline PCR & Polymerase chain reaction \\
\hline $\mathrm{PhSeT}$ & Phenylselenyl-modified thymidine \\
\hline PTM & Post-translational modification \\
\hline RNAP & RNA polymerase \\
\hline SDS-PAGE & Sodium dodecyl sulfate polyacrylamide gel electrophoresis \\
\hline ssDNA & single-stranded DNA \\
\hline SP & Stationary phase \\
\hline TEV & Tobacco etch virus protease \\
\hline TopA & Topoisomerase I \\
\hline UV & Ultraviolet \\
\hline
\end{tabular}




\section{INTRODUCTION}

\section{I.1 DNA TOPOLOGY}

Double helical deoxyribonucleic acid (DNA) is fundamental for every living organism. The highly ordered structure of the double helix is formed by two base-paired complementary strands that follow a right-handed helical path about a central axis. The discovery of DNA structure by Watson and Crick in 1953 is often marked as the birth of modern molecular biology (Watson and Crick, 1953). DNA is a very flexible and dynamic molecule during cellular processes. A typical double helix DNA molecule consists of two intertwined complementary polynucleotide chains. B-DNA is the biologically predominant form of DNA structure (Mirkin, 2001). It consists of a right-handed helical conformation, and the detailed structure is dependent on the local sequence of nucleotides, DNA binding proteins and DNA-centered processes (Travers and Muskhelishvili, 2015). The fact that the double helix DNA strands are continuously intertwined gives rise to topological properties of DNA structures.

This intertwining of DNA double helix introduces supercoils and changes gene expression. Winding DNA in a right-handed direction as the helix generates positive supercoiling, which is also called over-wound DNA, whereas winding in a left-handed direction generates negative supercoiling, called under-wound DNA (Figure 1) (Baranello et al., 2012; Mirkin, 2001). DNA transactions in prokaryotes and eukaryotes require DNA to be unwound allowing the readout of DNA recognition sequences by proteins (Freeman and Garrard, 1992; Travers and Muskhelishvili, 2015). One possible solution is the unhindered rotation at the free end of DNA if the other end of DNA molecule is fixed. 
Another possible solution is the introduction of DNA strand breakage to allows DNA relaxation through the DNA strand break.

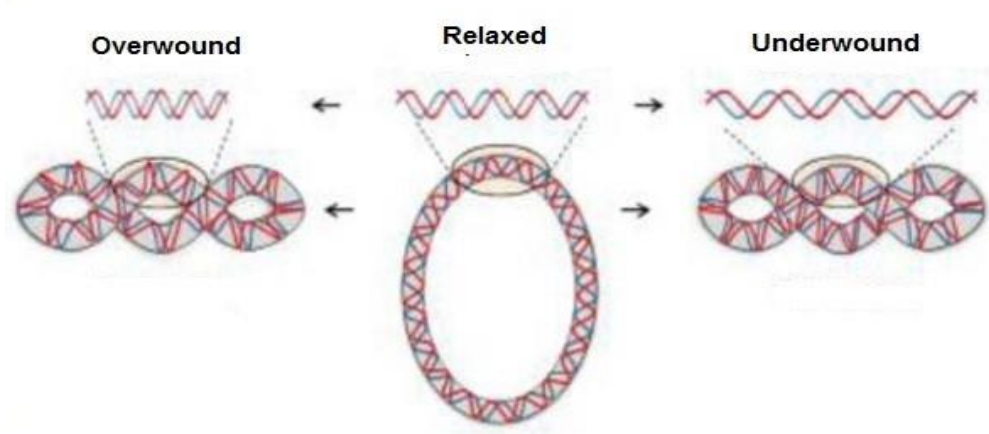

Figure 1 Structures of negative and positive DNA supercoilings (Baranello et al., 2012)

In reality, DNA molecules are generally very long and can form topological domains. For instance, numerous bacteria, mitochondria, viruses and chloroplasts have circular duplex molecules (Griffiths, 1999; Vologodskii, 2016). The circular DNA structure is a canonical example of a topological domain. In the present case, the DNA duplex is closed, obviously the circular DNA has no open end. Another example of topological domain is found in eukaryotic DNA. Even though eukaryotic chromosomes are linear overall, the linear structures consist of large DNA loops attached to the nuclear matrix. In other word, eukaryotic DNAs are topologically equivalent to circular DNAs (Dixon et al., 2012; Gilbert and Allan, 2014; Roca, 2011a). Thus, topological domains and the very large size of DNA molecules make the free ends rotation impossible for relaxation of the DNA duplex.

Another problem is that the DNA topological stresses are dynamically involved in many DNA-centered processes, including DNA replication, repair, recombination, and 
transcription (Keszthelyi et al., 2016; Liu and Wang, 1987; Postow et al., 2001; Roca, 2011a). When the tracking of molecular machinery, such as replication complexes or transcription complexes, is moving through the DNA, the forward double helix is required to be unwound in order to accommodate this motion. Moreover, the DNA-centered processes can cause topological stresses by themselves. Opening up the double helix without changing the total intertwines between strands will introduce localized topological stress in DNA. As the replication bubble progresses along the DNA, it forces double strands apart which builds up overwound DNA ahead and leaves intertwined daughter strands behind (Figure 2A) (Keszthelyi et al., 2016; Schoeffler and Berger, 2008). If such topological stress is not resolved and accumulates, replication bubble progression will be arrested leading to DNA breaks (Tuduri et al., 2009). Replication and recombination processes can generate knots and more complex mechanical interlocking such as catenanes to chromosomes (Champoux, 1980). During transcription process, the DNA template is unwound allowing transcription complex to get access to the coding strand. This also

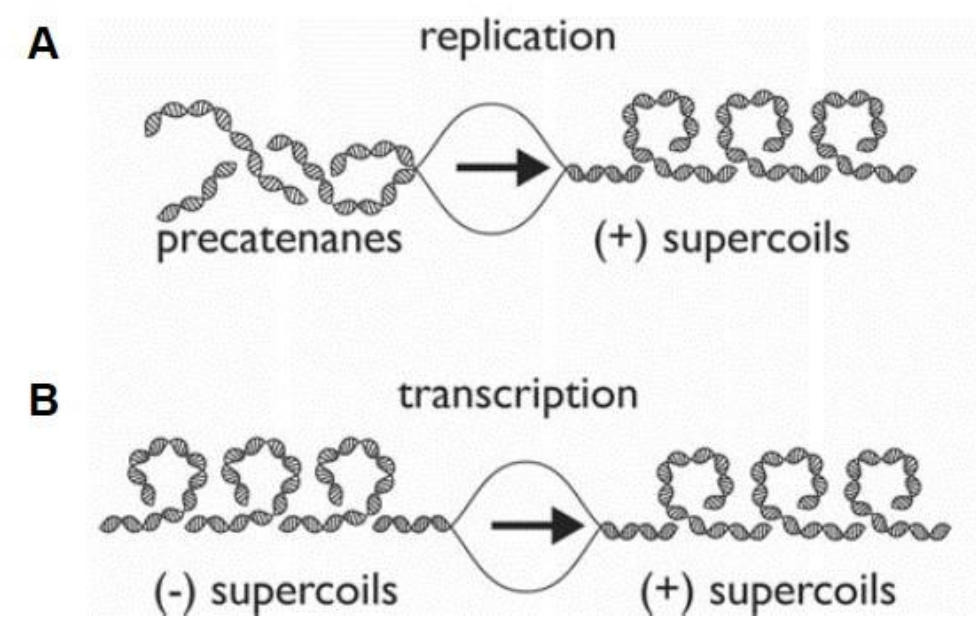

Figure 2 Topological problems during DNA replication and transcription (Schoeffler \& Berger, 2008) 
causes compensatory over-winding of DNA ahead and under-winding of DNA behind the transcription bubble (Figure 2B) (Schoeffler and Berger, 2008). Transcription-generated topological stress inhibits polymerase activity, thus affecting gene expression (Roca, 2011b).

The solution of resolving unfavorable topological stresses came to light with the discovery of the first DNA topoisomerase in 1971 (Wang, 1971). Since then, a special group of enzymes which carry out similar functions or follow similar mechanisms have been discovered (Corbett and Berger, 2004). These enzymes introduce transient DNA breaks and allow the single-stranded DNA or double-stranded DNA to pass through each other to resolve the DNA topological problems. The simple and elegant chemistry of transesterification to overcome DNA topological barriers makes DNA topoisomerases the "true magicians" in the DNA world (Wang, 2002).

\section{I.2 DNA TOPOISOMERASES}

\section{I.2.1 Classification of DNA topoisomerases}

The DNA topoisomerases cleave single- or double- stranded DNA through the nucleophilic attack by an active site tyrosine, conserved in all topoisomerases, to the phosphodiester backbone, creating a covalent phosphotyrosyl linkage with DNA (Figure 3). Depending on how many strands are cleaved during catalytic reactions, topoisomerases are classified into two types: type I topoisomerase which cleaves single strand at a time, type II topoisomerase which cleaves double strands at a time (Champoux, 2001; Wang, 2002). Subfamilies of these two types of enzymes are further divided into type IA, type IB, 
type IC, type IIA and type IIB, based on their structural and mechanistic similarities (Table $1)$.

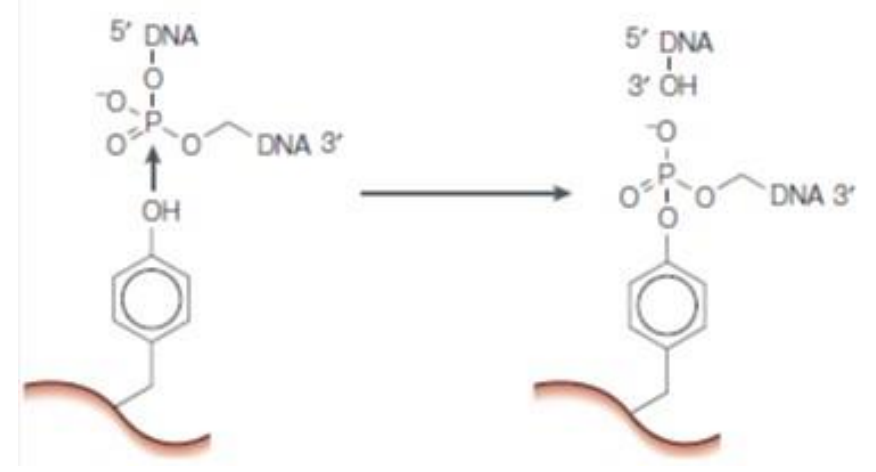

Figure 3 Catalysis of DNA transient breakage by DNA topoisomerases (Wang, 2002)

\begin{tabular}{|l|l|}
\hline Subfamilies & Representative members \\
\hline Type IA & $\begin{array}{l}\text { Bacterial and archael DNA topoisomerase I and III, } \\
\text { Yeast DNA topoisomerase III, } \\
\text { Mammalian DNA topoisomerase III } \alpha \text { and III } \beta .\end{array}$ \\
\hline Type IB & $\begin{array}{l}\text { Eukaryotic DNA topoisomerase I, } \\
\text { Mammalian mitochondrial DNA topoisomerase I, } \\
\text { Poxvirus topoisomerase. }\end{array}$ \\
\hline Type IC & Methanopyrus topoisomerase V (archael) \\
\hline Type IIA & $\begin{array}{l}\text { Bacterial gyrase and DNA topoisomerase IV, } \\
\text { Phage T4 DNA topoisomerase, } \\
\text { Yeast DNA topoisomerase II, } \\
\text { Mammalian DNA topoisomerase II } \alpha \text { and IIB. }\end{array}$ \\
\hline Type IIB & \begin{tabular}{l} 
Sulfolobus shibatae DNA topoisomerase VI \\
\hline
\end{tabular}
\end{tabular}

Table 1 The subfamilies and representative members of DNA topoisomerases 
Type IA topoisomerase, first discovered by Wang in 1970s, could relax negatively supercoiled DNA by cleaving a single strand of DNA, forming a covalent complex at the 5'-end and passing another strand of DNA in an ATP-independent manner (Figure 4A). Type IA includes bacterial and archaeal topoisomerase I and topoisomerase III, yeast topoisomerase III and mammalian DNA topoisomerase III $\alpha$ and III $\beta$. Type IB, discovered by Champoux and Dulbecco, could relax both positive and negative supercoils by cleaving single strand of DNA, forming a covalent comlex at the 3'-end (Figure 4B) (Champoux and Dulbecco, 1972). Type IB includes eukaryotic topoisomerase I, mammalian mitochondrial DNA topoisomerase I and poxvirus topoisomerase (Corbett and Berger, 2004). Type IC is functionally similar to type IB in that the enzyme is covalently linked at the 3' end of DNA and relaxes both positive and negative supercoils. Type IC only consists of Methanopyrus topoisomerase V in archaea (Gadelle et al., 2003).

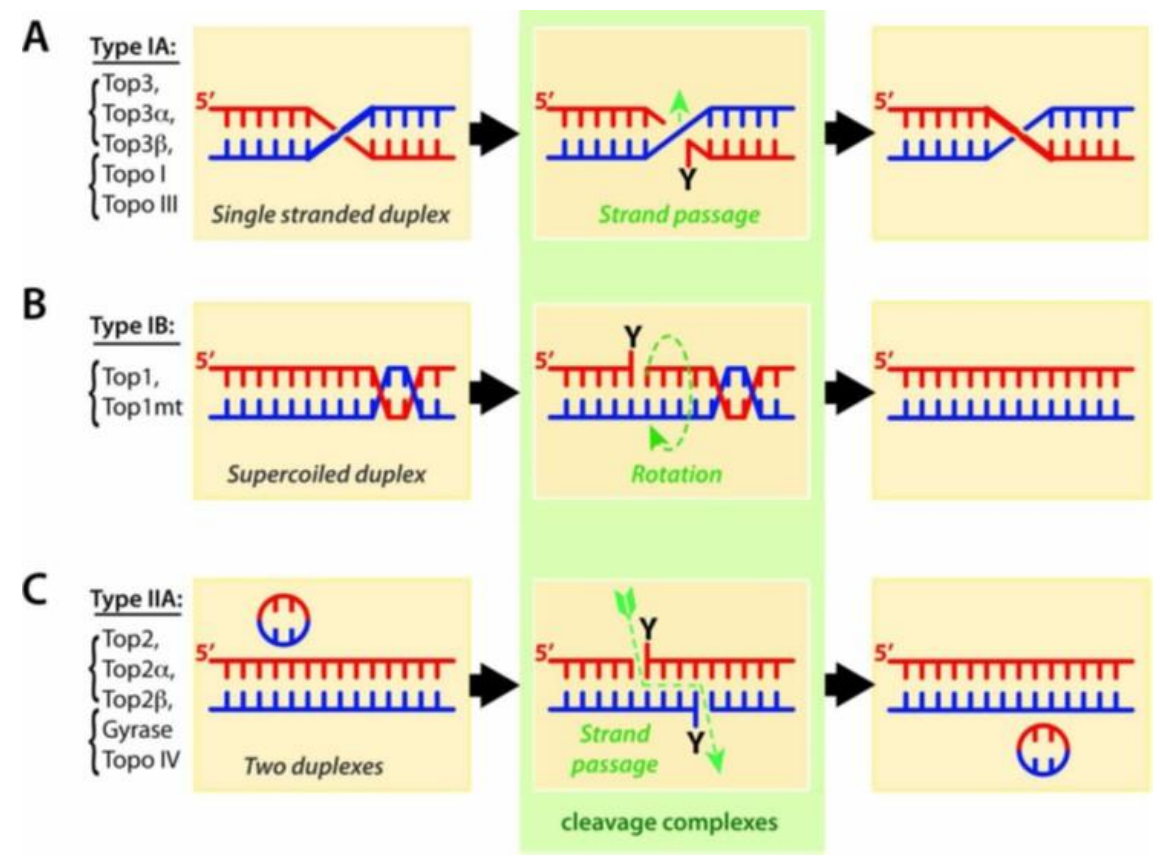

Figure 4 Catalytic mechanisms of topoisomerases (Pommier, 2013) 
Type IIA topoisomerase, discovered by Gellert, transports one double strand DNA through another in an ATP-dependent manner (Figure 4C) (Gellert et al., 1976). Type IIA includes bacterial gyrase, bacterial topoisomerase IV, viral and bacteriophage topoisomerase II, eukaryotic topoisomerase II. Topo II and topo IV both relax negative and positive supercoils, but topo IV can catalyze the relaxation of positive supercoils with higher efficiency. Gyrase can relax positive supercoils and can also introduce negative supercoils. Type IIB topoisomerase, discovered by Bergerat, shares a structure similarity with type IIA topoisomerase in the catalytic domains (Bergerat et al., 1994). Type IIB enzymes are present in most archaea and higher plants (Corbett and Berger, 2003; Hartung and Puchta, 2001). Only topo VI belongs to this subfamily, which shares a similar catalytic profile with type IIA but with distinct sequence and overall structure from other topoisomerases (Nichols et al., 1999). Both type IIA and IIB topoisomerases form covalent linkage to the cleaved DNA at its 5' end and require ATP and $\mathrm{Mg}^{2+}$ for activity.

\section{I.2.2 Functions of DNA topoisomerases}

In DNA replication, the roles of topoisomerases are well understood. Topoisomerases have been reported to play important roles in the following three replication phases: replication initiation, replication elongation, and replication termination (Vos et al., 2011). The replication initiation starts by opening of a short region at replication origins. In E. coli, negative supercoiling at the start site is required, the localized negative supercoiling is regulated by the opposite effects of DNA gyrase and topo IA (Kaguni and Kornberg, 1984). Moreover, topoisomerase I is involved in preventing erroneous initiation on R loops (Drolet et al., 1995). Similar function has been found in eukaryotes as well that 
Topo I is required to resolve R loop with RNase $\mathrm{H}$ at the replication origin (Stuckey et al., 2015). Replication fork progression leads to the accumulation of positive supercoiling ahead. In bacteria, the accumulated positive supercoils ahead can be resolved by DNA gyrase and topo IV. Replication elongation also leads to the formation of intertwined DNA strands behind. The intertwining of DNA duplex, also termed as precatenane in bacteria, will cause abnormal DNA segregation if left unresolved (Peter et al., 1998). Type II topoisomerases play an important part in resolving such topological linkages (Figure 5) (Baxter and Diffley, 2008; Holm et al., 1985). Topoisomerases have also been discovered to play a part in replication termination. In E. coli, type IA topoisomerase coordinates with terminus site-binding protein (Tus) to block the progression of the replicative helicase DnaB (Valjavec-Gratian et al., 2005).

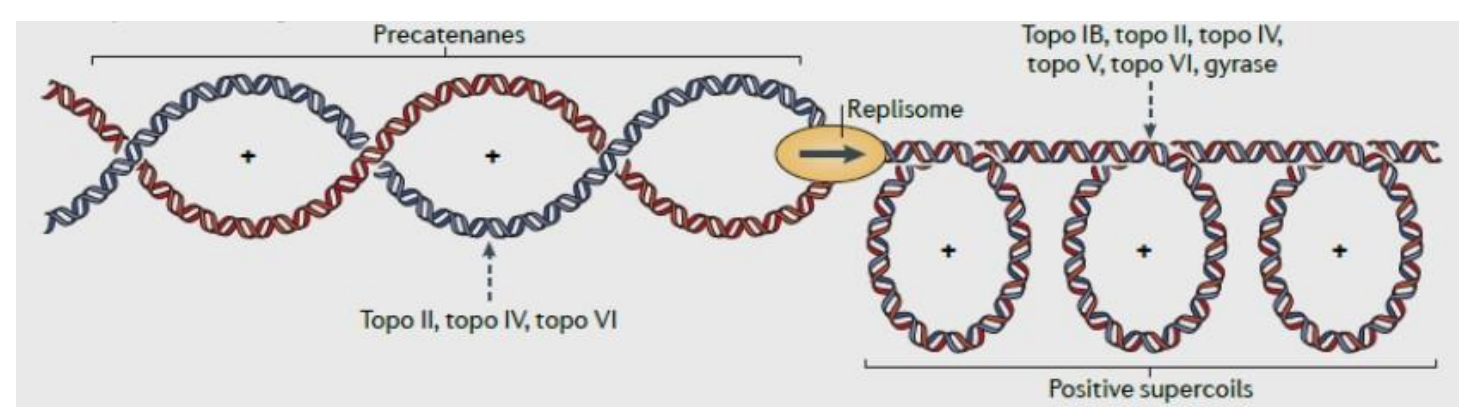

Figure 5 Topoisomerase functions during DNA replication (Vos et al., 2011)

Similar to DNA replication, transcription introduces DNA topological stress. Progression of transcription bubble generates positive supercoils ahead and negative supercoils in its wake (Drolet et al., 1994). These unfavorable supercoiling can cause improper gene expression if left unresolved. Eukaryotic type IB and bacterial type IIA are responsible for relaxation of positive supercoils ahead of transcription bubble. Type IA and 
type IB are involved in removal of negative supercoils (Figure 6) (Schoeffler and Berger, 2008). At the transcription bubble, longer nascent RNA might hybridize with its template DNA strand, thus leaving the non-templated strand single stranded. This three-stranded structure can lead to deleterious outcome on genome integrity. Topoisomerase I helps to repress transcription-mediated R-loop formation in bacteria (Usongo and Drolet, 2014).

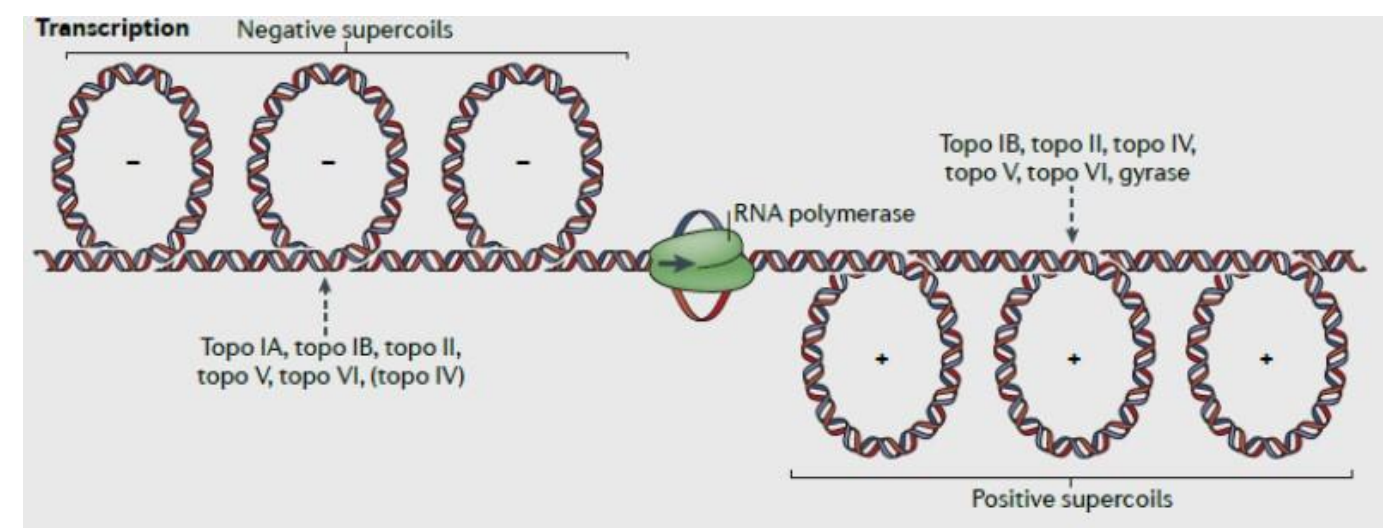

Figure 6 Topoisomerase functions during DNA transcription (Vos et al., 2011)

Another important function of topoisomerases is that these enzymes are important in the formation and management of double-stranded DNA breaks in meiotic recombination and DNA repair. During meiotic recombination, SPO11, homologue of topo VI, cleaves double-stranded DNA allowing strand passage for homologous recombination (Fukuda et al., 2008). Evidence suggested the participation of topoisomerase in recombination repair that participation of type IA in homologous recombination: topoisomerase breaks up double Holliday junction and may help recruit other homologous recombination proteins to the DNA breakage (Cejka et al., 2010; Harmon et al., 2003). 


\section{I.2.3 Structure and mechanism of $E$. coli type IA topoisomerases}

At least one type IA topoisomerase exists in each bacterial species (Forterre et al., 2007). Type IA topoisomerases are able to relax negative supercoiled DNA efficiently. $E$. coli type IA topoisomerases includes two well-studied enzymes: E. coli topoisomerase I and E. coli topoisomerase III. All type IA topos share a padlock structure in shape, with a cavity in the center surrounded by four major domains (Lima et al., 1994; Mondragon and DiGate, 1999). Domain I contains Rossmann-like TOPRIM fold, a parallel, four- or fivestranded $\beta$ sheet sandwiched by $\alpha$ helices. A cluster of three acidic residues located in domains I is responsible for the binding of $\mathrm{Mg}$ (II) which is required for DNA religation (Zhu and Tse-Dinh, 2000). Domain II consists of an all- $\beta$ fold arching over other domains, forming the central cavity. The active site tyrosine is located in the WHD fold in domain III. The active tyrosine in domain III is responsible for the G-strand breakage reaction. Domain IV, together with Domain I, III, forms a positively charged binding groove for Gstrand DNA. During relaxation, the cleaved 5' end of DNA is covalently linked to the active site tyrosine, and the 3 ' end of DNA is bound non-covalently to domain IV. The interface of these three domains controls DNA access into the enzyme active site. The conserved catalytic core for type IA topoisomerase is formed by four major domains (domain I-VI) in the N-terminal region, referred to as the "topofold" (Figure 7B). The Cterminal region of type IA topos is less conserved. For instance, E. coli topoisomerase I has a 4-Cys zinc ribbon domain and zinc ribbon-like domain for T-strand DNA binding, assisting the single-strand DNA movement for full catalytic activity (discussed later) (Ahumada and Tse-Dinh, 1998, 2002; Tan et al., 2015; Zhu and Tse-Dinh, 1994). E. coli 
topo III contains the decatenation loop at the edge of central cavity, but it lacks the zinc binding domain (Figure 7A) (Li et al., 2000).

A

E. coli topoisomerase I

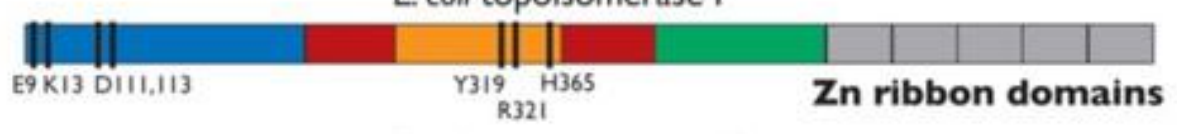

E. coli topoisomerase III

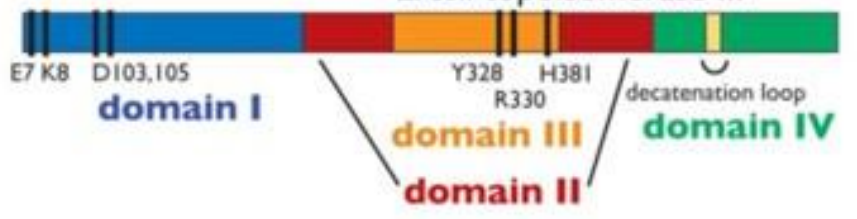

B

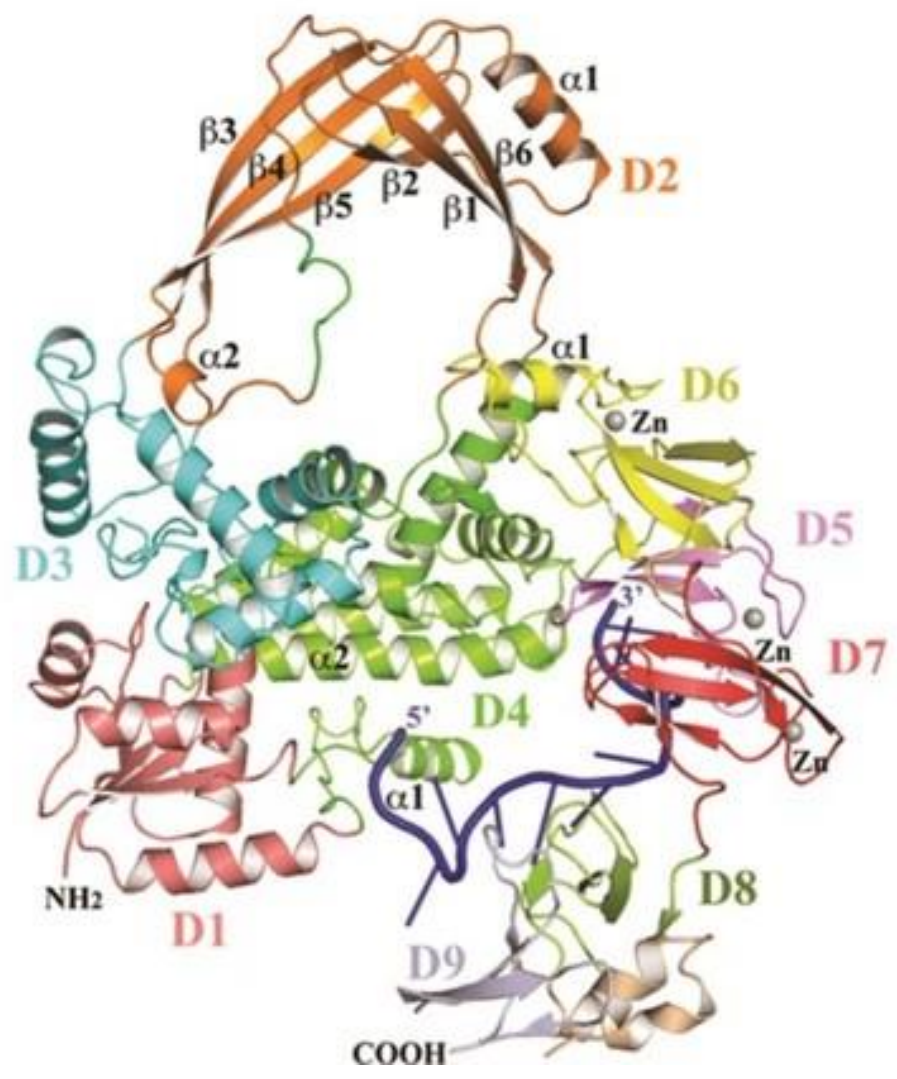

Figure 7 Organization and structure of type IA topoisomerases (Schoeffler and Berger, 2008, Tan et al., 2015) 
Type IA topoisomerases is proposed to catalyze DNA relaxation through an enzyme-bridged strand passage mechanism (Figure 8). The active site tyrosine is located at the interface of domain I and domain III. With the help from domain II and domain IV, the catalytic center opens and closes the G-segment DNA upon binding. During the catalysis, two conformations of the enzyme are involved: an open conformation and a closed conformation. First, the G-strand DNA binds to the enzyme (Figure 8, step 1). The binding of G-strand activates domain III of the enzyme. Domain III undergoes a conformational change allowing the active site tyrosine located in domain II to cleaves the G-strand to form a covalent 5' phosphotyrosyl bond with the DNA, while the 3' end of the G-strand is bound to domain IV (Figure 8, step 2). After cleavage, domain III with the DNA strand covalently bound on it is moved away from domain I and domain IV to form a DNA gap for the T-strand DNA to pass through. The hinge domain in domain II and domain III may contribute to the conformational change (Figure 8, step 3). Once the Tstrand is moved through the DNA gap, the protein gate is closed and the DNA gap is religated (Figure 8, step 4 and step 5). The enzyme must undergo another conformational change to release the relaxed DNA (Figure 8, step 6 and step 7). After the relaxation is complete, the enzyme can carry out another round of relaxation with the same or new DNA substrate (Viard and de la Tour, 2007). 


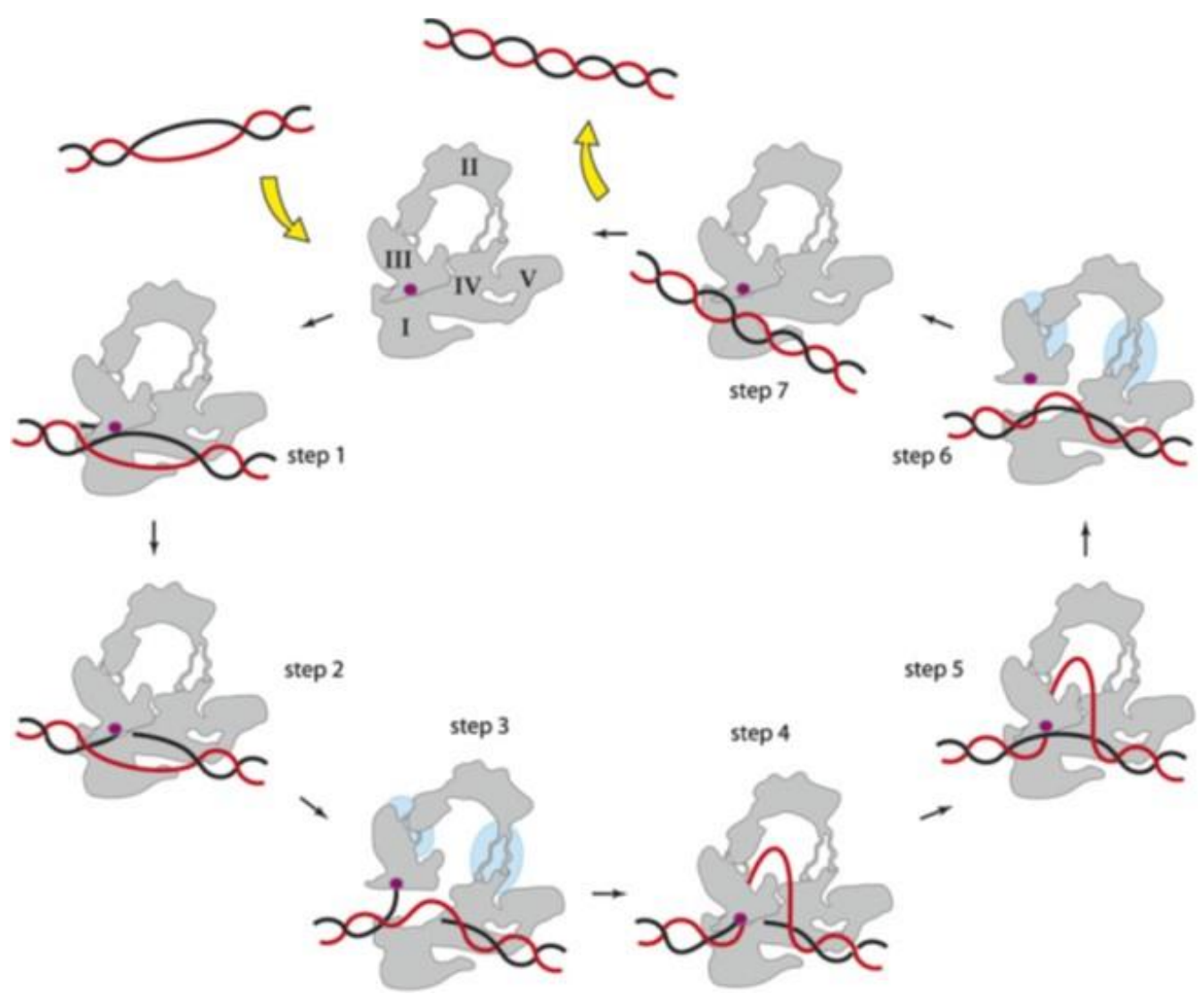

Figure 8 Enzyme-bridged strand passage mechanism of type IA topoisomerase (Viard and de la Tour, 2007)

Even though some of the overlapping functions of topoisomerase I and III have been mentioned previously, the two type IA topoisomerases appear to be optimized to have their own specific functions. Indeed, topoisomerase I is an essential enzyme for bacterial survival and is very efficient in relaxing negative supercoiling. While topoisomerase III is more efficient for decatenating the precatenanes generated behind the replication bubble than relaxing negative supercoiling (DiGate and Marians, 1988; Hiasa et al., 1994) 


\section{I.2.4 The significance of studying $E$. coli topoisomerase I}

Studying E. coli topoisomerase I is significant in three aspects. First of all, it will help to elucidate the systematic regulation on DNA topology and gene expression under various conditions. E. coli topoisomerase I was first discovered capable of relaxing supercoils. The enzyme is essential for DNA supercoiling regulation. The relaxation action of topoisomerase I and supercoiling action of DNA gyrase are the two major determinants in maintaining the balance of global DNA supercoiling. Any mutation of topoisomerase I altering the proper relaxation activity leads to growth defects. The growth defect caused by topoisomerase I mutations that reduces its activity can be compensated by introducing another mutation on DNA gyrase (DiNardo et al., 1982; Pruss et al., 1982). Secondly, studying E. coli topoisomerase I may help the investigations of other type IA topoisomerases. Sequence analysis and crystal structures of type IA topoisomerases revealed that all type IA topoisomerases share the common topofold structure formed by several conserved domains. The active site structure for DNA cleavage and rejoining is evolutionarily conserved (Baker et al., 2009). The zinc ribbon domain found in E. coli topoisomerase I is also present in human type IA topoisomerases TOP $3 \alpha$ and TOP $3 \beta$, that play a role in preventing genomic instability (Seki et al., 2006). Finally, studying E. coli topoisomerase I will contribute to drug discovery efforts aiming to identify new antibacterial compounds targeting bacterial topoisomerase I. Human type IB topoisomerase, type IIA topoisomerase and bacterial type IIA topoisomerase are welldeveloped drug targets for anticancer and antibacterial drugs (Drlica and Malik, 2003; Giles and Sharma, 2005; Pommier, 2006). Bacterial topoisomerase I is a potential target 
for new antibacterial drugs because of the existence of the enzyme in all bacterial species (Tse-Dinh, 2009). Inhibitors that trap the bacterial topoisomerase I covalent complex should be bactericidal. So far, the emergence of "superbugs" that are resistant to all currently available antibiotics presents an urgent need for discovery of new antibacterial compounds. Elucidating the function and regulation of E. coli topoisomerase I will potentially contribute to the drug discovery targeting topoisomerase I to fight against the global public health threats of antibiotics resistance.

\section{I.2.5 E. coli Topoisomerase I is acetylated}

Proteomics studies have revealed multiple lysine sites on E. coli topoisomerase I as targets of lysine acetylation (Yu et al., 2008; Zhang et al., 2009a). Moreover, the proteome microarray assay identified a strong interaction between a deacetylase and E. coli topoisomerase I suggesting the importance of regulation of lysine acetylation in E. coli topoisomerase I (Liu et al., 2014). Similarly, eukaryotic topoisomerases have been found to be post-translationally modified by acetylation (Choudhary et al., 2009), phosphorylation (Wells et al., 1994), sumoylation (Mao et al., 2000) and ubiquitylation (Mao et al., 2001) that can alter the protein activity and localization.

\section{I.3 POST-TRANSLATIONAL MODIFICATION}

\section{I.3.1 Overview of post-translational modification}

Living cells are constantly exposed to external stimuli and consequently have to develop strategies to rapidly respond to environment. Multiple levels of regulation are used by cells for survival including regulating gene expression and protein post-translational 
modification. Compared to the relatively slow regulation of gene expression level and translational level, posttranslational modification (PTM) of proteins is very quick and is considered to be the major mechanism of regulation. Currently, 481 PTMs have been listed in the Uniprot database broadly distributed in eukaryotes, prokaryotes and archaea (The UniProt Consortium, 2017). Examples of PTMs include phosphorylation, acetylation, ubiquitination, glycosylation, lipidation, methylation, proteolysis and others. These modifications diversify and extend protein functions beyond gene expression. 'Mod-form distribution' of PTM was introduced to describe the state that one protein can be altered by multiple PTMs at multiple sites eliciting distinct biological output. Therefore, complex biological processes can be orchestrated dynamically in response to the environment at the system level (Cain et al., 2014; Prabakaran et al., 2012). The PTM of proteins generate a multitude of modification of protein properties, including altering the protein structure, protein complex assembly, protein functional activity, interactions with other biomolecules and cellular localization. These modifications are crucial regulators of cellular physiology in many cellular processes such as signaling cascades, gene transcription, cell division and metabolism (Knorre et al., 2009). Defects in PTMs can cause various developmental disorders and human diseases (Karve and Cheema, 2011).

\section{I.3.2. Acetylation}

One of the most abundant posttranslational modification is lysine acetylation. The modification take place either at the $\varepsilon$-amino group of internal lysine residues, or at the $\alpha$ amino group at the N-terminus of a protein (Figure 9). The direct effect of acetylation is that the removal of the positive charge of the amino acid would cause a redistribution of 
the overall charge in a protein, also it would change the local hydrophobicity and steric effects of the side chain of the modified amino acid.

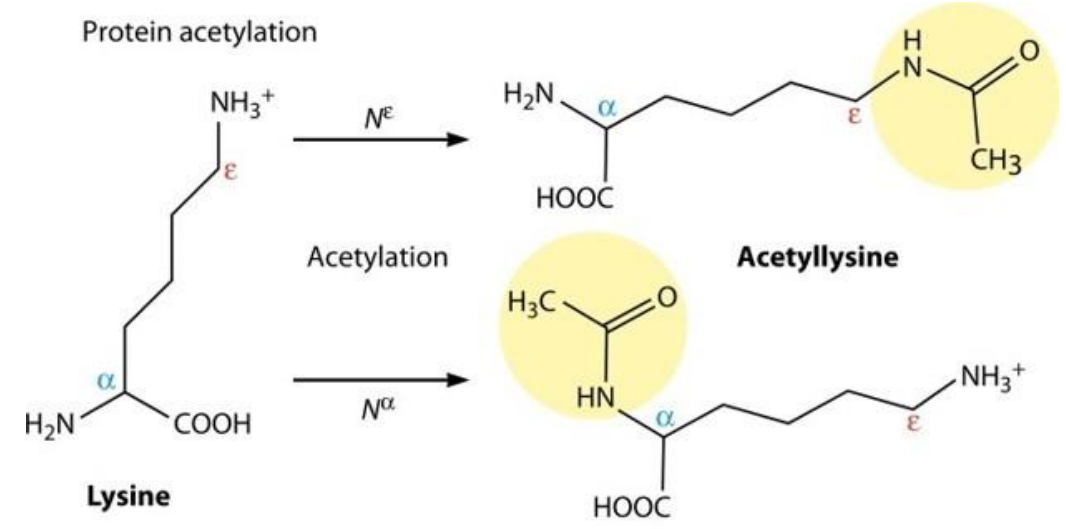

Figure 9 Schematic of $\mathbf{N}^{\varepsilon}$ - and $\mathbf{N}^{\alpha}$-acetylation (Hentchel and Escalante-Semerena, 2015)

\section{I.3.2.1 $\mathrm{N}^{\alpha}$-acetylation}

$\mathrm{N}$-terminal acetylation, namely $\mathrm{N}^{\alpha}$-acetylation, refers to the addition of an acetyl group from acetyl donor to the $\alpha$-amino group of $\mathrm{N}$-terminus of a protein. Based on spatiotemporal occurrence, $\mathrm{N}^{\alpha}$-acetylation is divided into co-translational acetylation or post-translational acetylation (Varland et al., 2015). About $80 \%-90 \%$ of human proteins and about $50 \%-60 \%$ of yeast proteins are co-translationally acetylated on the $\mathrm{N}^{\alpha}$-terminus (Arnesen et al., 2009). $\mathrm{N}^{\alpha}$-acetylation can occur on $\mathrm{N}$-terminal starting methionine residues, or on newly exposed N-terminal amino acid residues in ribosomes (Aksnes et al., 2015). Recently, growing evidence has shown that $\mathrm{N}^{\alpha}$-acetylation occurs post-translationally on several proteins (Helsens et al., 2011; Van Damme et al., 2011), suggesting its role in a more complex system. $\mathrm{N}^{\alpha}$-acetylation is considered irreversible, and requires $\mathrm{N}$-terminal 
acetyltransferases (NATs) to bind the acetyl donor acetyl coenzyme A (acCoA), then transfer acetyl group to the protein N-terminus to be modified. The NAT family of acetyltransferases belongs to the GNAT superfamily (Gcn5-related N-acetyltransferases) sharing conserved sequence motifs of $\mathrm{Q} / \mathrm{RxxGxG} / \mathrm{A}$ in the acetyl-CoA binding sites (Aksnes et al., 2015). So far, six subtypes (NatA to NatF) of NAT family have been identified in mammalian cells, and five subtypes have been found in yeast (NatA to NatE). These subtypes have distinct substrate specificities. The NatA enzyme acetylates Nterminal Ser/Ala/Thr/Gly/Val/Cys following the cleavage of methionine. All other Nat subtypes acetylate $\mathrm{N}$-terminal methionine. Their substrate specificities are determined by the subsequent amino acid. NatB acetylates N-terminal methionine which is followed by Asp/Glu/Asn (Met-Asp/Glu/Asn). NatC acetylates N-terminal Met-Ile/Leu/ Phe/Trp. NatD acetylates histone $\mathrm{H} 2 \mathrm{~A}$ and $\mathrm{H} 4$. NatE and NatF, sharing overlapping substrate specificities with NatC, can potentially acetylate $\mathrm{N}$-terminal Met-Met/Tyr/Lys. Overall, $\mathrm{N}^{\alpha}$-acetylation is highly common in eukaryotes but very rare in bacteria. Few NAT acetyltransferases have been found in E. coli, namely RimJ, RimI, and RimL, which can only acetylate a small number of ribosomal proteins (Charbaut et al., 2002; Gordiyenko et al., 2008).

Although $\mathrm{N}^{\alpha}$-acetylation has been discovered many years ago, the functional roles of $\mathrm{N}$-terminal acetylation emerged only recently. $\mathrm{N}^{\alpha}$-acetylation blocks further ionization and other modifications through neutralization of the positive charge of the free $\alpha$-amino group. N-terminal acetylation plays important roles in several cellular processes. Nterminal acetylation is related to the $\mathrm{N}$-end rule pathway to decrease protein stability and cause degradation (Giglione et al., 2003; Shemorry et al., 2013; Varshavsky, 2011). One 
the one hand, $\mathrm{N}^{\alpha}$-acetylation may protect protein from proteasomal degradation or inhibit signaling events mediated by ubiquitylation. It marks proteins for certain ubiquitin ligase recognition which causes subsequent degradation (Scaglione et al., 2013; Tatham et al., 2013). In addition, $\mathrm{N}$-terminal acetylation is associated with protein subcellular location in cytoplasm, protein complex formation and protein folding (Aksnes et al., 2015).

\section{I.3.2.2 $\mathrm{N}^{\varepsilon}$-acetylation}

$\mathrm{N}^{\varepsilon}$-acetylation was first reported on histone about fifty years ago. Actually, it was first described by Allfrey and his colleague as acetylation on N-terminal amino acid (Allfrey et al., 1964; Allfrey and Mirsky, 1964). Later, it was discovered to be the acetylation on $\varepsilon$-amino group of a lysine residue (Gershey et al., 1968). Since then, intensive research has discovered various $\mathrm{N}^{\varepsilon}$-lysine acetylated proteins and associated functions from mammals to bacteria. In contrast to $\mathrm{N}^{\alpha}$-acetylation, $\mathrm{N}^{\varepsilon}$-lysine acetylation is a reversible process, which makes it important for cells to switch on/off certain cellular pathway. In eukaryotes, lysine acetylation is regulated by lysine acetyltransferases (KATs), lysine deacetylase (KDACs), and acetyl-lysine binding proteins. KATs catalyze the

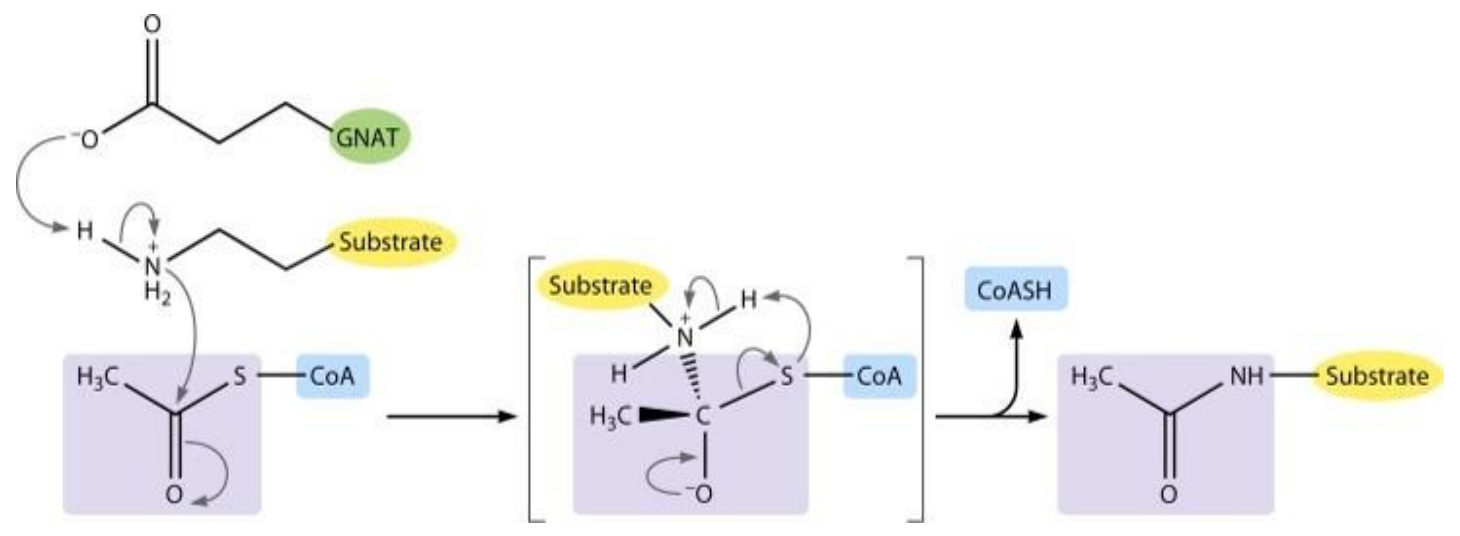

Figure 10 Acetylation mechanism of KATs (Hentchel and Escalante-Semerena, 2015) 
deprotonation reaction of the target lysine residue and promotes the nucleophilic attack on the carbonyl carbon of acetyl-CoA (Figure 10). Up to twenty KATs have been identified so far in human carrying out acetylation with specific targets. Lysine deacetylases (KDACs) also named as histone deacetylases (HDACs) are mechanistically divided into two groups: $\mathrm{Zn}^{2+}$ dependent HDACs, and NAD ${ }^{+}$dependent sirtuins, the later one is also found in bacteria. The $\mathrm{Zn}^{2+}$ dependent HDACs use histidine residue coordinated with the $\mathrm{Zn}$ (II) ion to activate a water molecule that triggers a nucleophilic attack on a carbonyl group of the acetyl-lysine (Figure 11A). The $\mathrm{NAD}^{+}$dependent sirtuins catalyzed deacetylation requires the binding of $\mathrm{NAD}^{+}$to the catalytic site followed by ADP-ribosylation and inversion of the configuration (Figure 11B) (Hentchel and Escalante-Semerena, 2015). Besides KATs

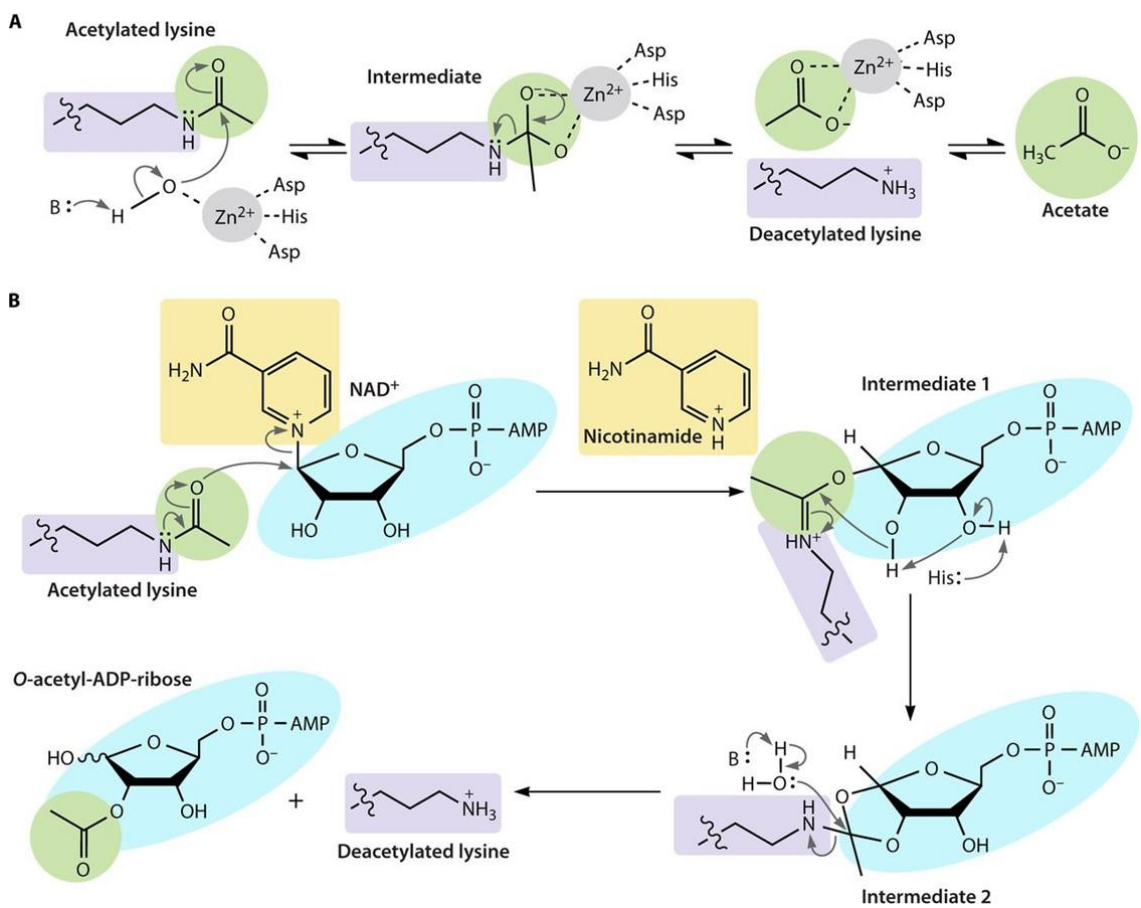

Figure 11 Deacetylation mechanisms of $\mathrm{Zn}^{2+}$ dependent HDACs and $\mathrm{NAD}^{+}$dependent sirtuins (Hentchel and Escalante-Semerena, 2015) 
mediated enzymatic acetylation, some mitochondrial proteins are nonenzymatically acetylated by acetyl-CoA, which is favored by high $\mathrm{pH}$ and high Ac-CoA concentration in mitochondria (Hosp et al., 2017; Wagner and Payne, 2013).

Generally, lysine is located on the hydrophilic surface of proteins. The positive charge of the $\varepsilon$-amino group on the lysine side chain contributes to protein stability and protein interactions through the formation of salt bridges and hydrogen bonds (Kang et al., 2016; Sokalingam et al., 2012). Thus, removal of the positive charge of lysine by acetylation will change protein structure and protein interactions with other biomolecules (Figure 12). In eukaryotes, lysine acetylation is best known for its role in epigenetic regulation of gene expression. Site-specific acetylation on histone interferes with histoneDNA and histone-histone interactions thereby disrupting histone assembly, nucleosomes formation and the folding of nucleosomes into higher-order chromatin (Eberharter and Becker, 2002; Tessarz and Kouzarides, 2014). Besides, acetylation promotes histone

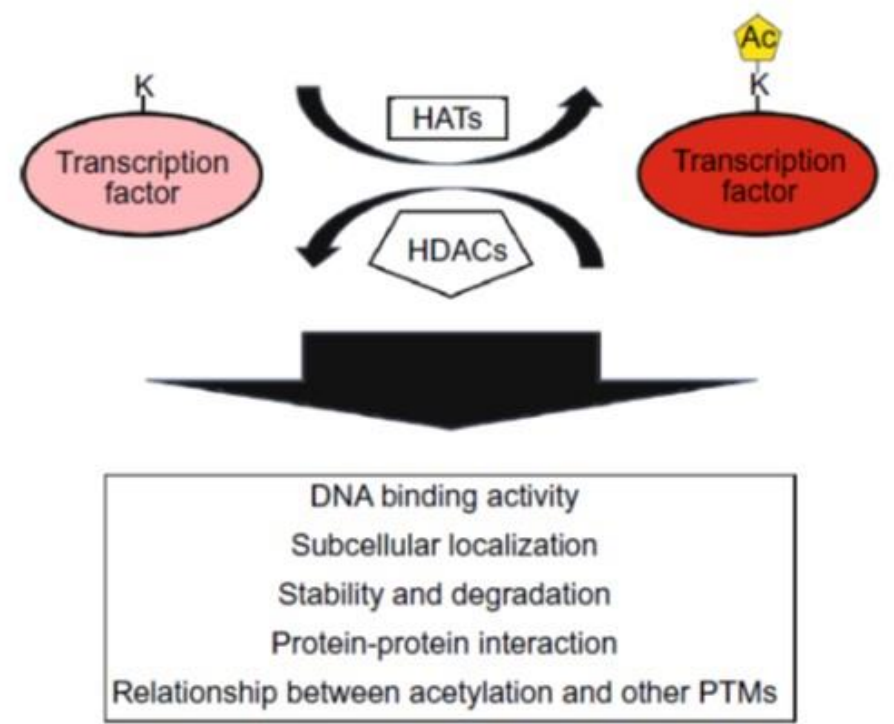

Figure 12 Functional consequences of lysine acetylation (Park et al., 2015) 
interaction with transcription factors (Harbison et al., 2004). Acetylation on some lysine residues of histone is required for the binding of transcription factors and bromodomaincontaining proteins, which are part of large complexes that modulate chromatin architecture (Filippakopoulos and Knapp, 2014; Owen et al., 2000). Cytoskeletal proteins are another crucial family acetylated by KATs. Acetylation on monomeric G-actin protein increases the stability of microfilamentous F-actin and decreases restructuring of F-actin (Zencheck et al., 2012). Acetylation on cortactin protein abolishes the binding of cortactin to F- actin, thus directly influences cell motility (Zhang et al., 2007; Zhang et al., 2009b). Lysine acetylation also plays an important role in p53 activation and interactions. Tumor suppressor $\mathrm{p} 53$ is the first discovered non-histone KAT substrate. Although the p53 protein is modified by several types of PTM, acetylation regulates p53 activation and subcellular localization through altering the interaction of $\mathrm{p} 53$ with its target proteins (Gu and Roeder, 1997; Tang et al., 2008). Acetylation is enriched in mitochondria proteins, presumably linked with high turnover of acetyl-CoA in mitochondrial compartment. Enzymatic acetylation by mitochondrial KATs and non-enzymatic acetylation favored by the $\mathrm{pH}$ of in actively respiring mitochondria play important roles in regulating mitochondrial protein functions and thus affecting metabolism (Drazic et al., 2016; Hosp et al., 2017). The high abundance of lysine acetylation in mitochondrial proteins implies the possible widespread existence of the modification in prokaryotes, given the evolutionary lineage of eukaryotic mitochondria from bacteria (Gray et al., 1999). 


\section{I.3.3 Discovery of $N^{\varepsilon}$-acetylation in bacteria}

Lysine acetylation has been well-known for its role in eukaryotes for many years. The observation of lysine acetylation has been expanded since year 2000 to bacteria with two lysine-acetylated proteins investigated, acetyl-CoA synthetase (Acs) (Starai et al., 2002) and chemotaxis protein CheY (Ramakrishnan et al., 1998). Since then, large-scale and high-throughput proteomics advances have identified a large number of putative acetylated proteins and acetylated lysine sites. Currently, bacterial acetylomes have been investigated in different bacteria species including Escherichia coli, Salmonella enterica, Bacillus subtilis, Pseudomonas aeruginosa, Rhodopseudomonas palustris, Erwinia amylovora, Vibrio parahemolyticus, Mycobacterium tuberculosis, Mycoplasma pneumoniae, Thermus thermophiles, Cyanobacterium synechocystis, Staphylococcus aureus, Streptomyces roseosporus, Leptospira interrogans, Geobacillus kaustophilus and Saccharopolyspora erythraea. These proteomic profiles suggested the diversity, the frequency and the physiological significance of protein acetylation in prokaryotes (Bernal et al., 2014; Ouidir et al., 2016). The immunoaffinity approach represents the most commonly used approach to identify acetylated lysine targets. This methodology is mainly consisted by four steps (Figure 13): (i) the protein lysate is digested by trypsin; (ii) tryptic peptides are subjected to affinity purification with immobilized anti-acetyllysine antibody; (iii) the isolated and enriched lysine-acetylated peptides are analyzed by nanoHPLC/MS/MS; (iv) peptide candidates are interpreted using specific software. The general goal of the global proteomic analysis is (i) to determine the spectrum of lysine acetylation targets; (ii) to identify lysine acetylation sites and provide cue of candidate proteins for 
future studies; (iii) to define biological processes potentially affected by lysine acetylation (Ouidir et al., 2016).

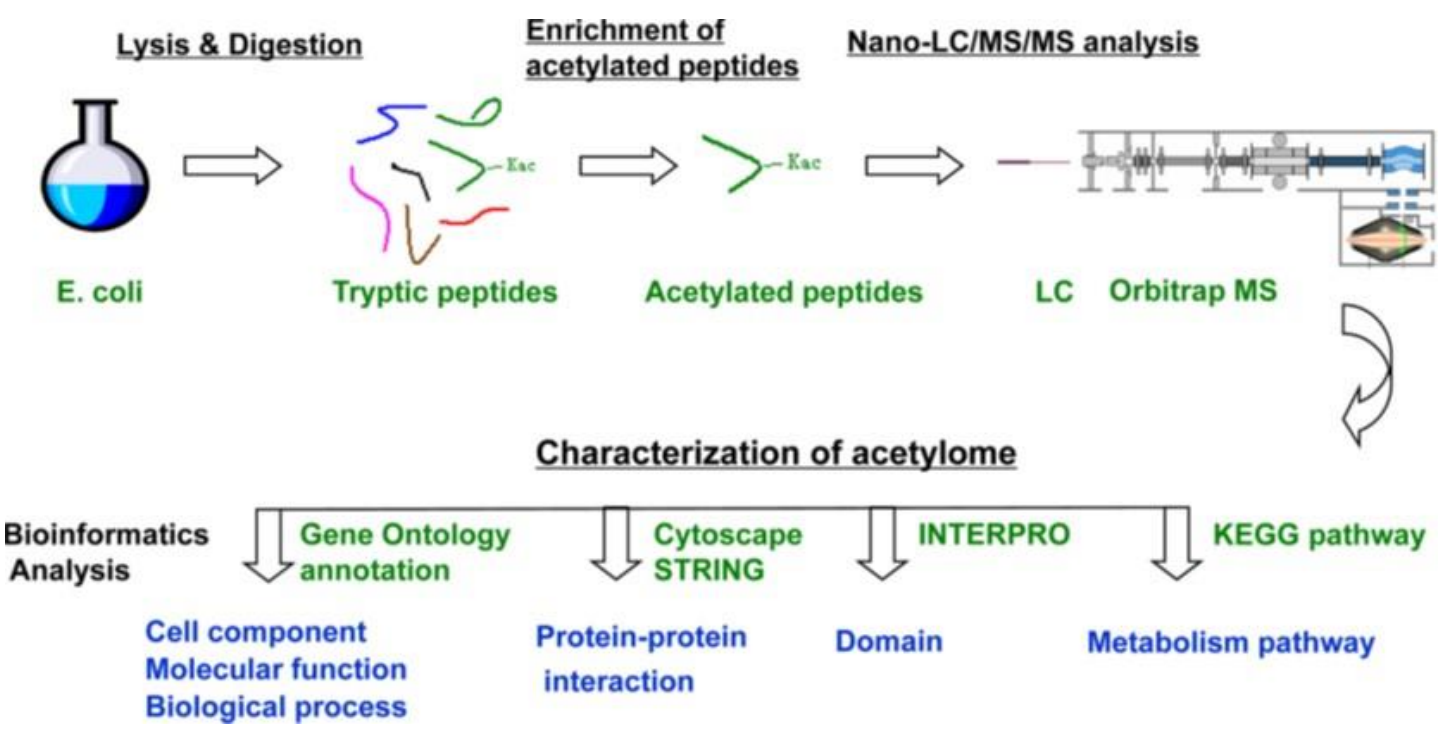

Figure 13 The analytical strategy and method for global profiling of lysine acetylation (Zhang et al., 2013a)

Protein acetylation profiling in E. coli was first characterized by $\mathrm{Yu}$ and his colleague in 2008 (Yu et al., 2008). They grew E. coli K-12 wild-type stain W3110 in nutrient rich Lysogeny broth (LB) medium to the mid-exponential phase (mid-EP) and stationary phase (SP), and harvested by centrifugation with the addition of $50 \mathrm{mM}$ nicotinamide (NAM) to the culture. The SP culture was re-inoculated and cultivated in fresh LB medium, then harvested with NAM to inhibit deacetylases. Cellular proteins were subjected to tryptic digestion after cell lysis. The tryptic digestion allows the mass spectrometry analysis of acetylated-lysine sites at the peptide level, which will make the analysis effectively easier because the digestion exposes lysine residues buried inside and reduces non-specific interactions. The immunoaffinity enrichment using an anti- 
acetyllysine antibody together with nano HPLC/MS/MS was used to analyze lysineacetylated peptides. Overall, this study identified 125 acetylated-lysine sites on 85 E. coli proteins, and acetylation of most lysine sites was growth phase dependent. Fifteen acetylated-lysine sites were found in both exponential and stationary phases, with 29 sites specific in exponential phase, and 82 sites were specific in stationary phase. The functional classification of the 85 acetylated proteins suggested the possible physiological significance of protein acetylation in diverse cellular functions. Most acetylated proteins were involved in protein synthesis, carbohydrate metabolism, nucleotide biosynthesis, amino acid biosynthesis, TCA cycle, transcription and cellular constituent. Other cellular functions included chaperone, detoxification, energy metabolism and fatty acid metabolism. This in-depth proteomic work and several following proteomics studies have emerged systematic analysis of protein acetylation in E. coli.

Zhang and his colleagues grew E. coil DH5 strain in LB medium to exponential phase (Zhang et al., 2009a). In addition to the immunoaffinity method described earlier, the combination of monoclonal and polyclonal anti-acetyllysine antibodies was used to improve the detection limit. One hundred and thirty-eight acetylation sites in 91 proteins were identified, among which 53\% acetylated proteins were involved in metabolism, 22\% were involved in translation. Others functions affected by acetylation included chaperones, stress response and transcription. The analysis confirmed the involvement of protein acetylation in diverse cellular functions reported previously (Yu et al., 2008).

A comprehensive profiling of protein acetylation in E. coli was reported later using a combined approach of immunoaffinity (Zhang et al., 2013a). The highly powerful and 
sensitive approach allowed mapping of the acetylome with more details. The detection range of acetylated lysine sites and acetylated proteins was significantly expanded, 1070 acetylated lysine sites in 349 acetylated proteins were identified. The characterization of acetylated proteins was not only based on biological processes, also from examination of cell components and molecular functions. It confirmed the findings in previous proteomic studies that most acetylated proteins were involved in metabolism, translation and transcription (up to $72 \%$ of total acetylated proteins). According to the gene ontology annotation, it was revealed that the majority of acetylated proteins were located in the cytoplasm, ribosome and membrane (about $86 \%$ ). Moreover, most of acetylated proteins were associated with biomolecule bindings and structural constituents of ribosome suggesting the potential association in protein function regulations.

Few studies have been done on the quantification aspect of lysine acetylated substrates. Stable isotope labeling with amino acids in cell culture (SILAC) was used to reveal changes of lysine acetylation abundance in E. coli (Figure 14). Weinert and his coworkers was first suggested the existence of acetyl-phosphate mediated nonenzymatic lysine acetylation in bacteria (Weinert et al., 2013). They have identified more than 8000 acetylation sites, and most acetylation accumulated in growth arrested cells affected by the level of intracellular acetyl-phosphate formed through glycolysis or acetate assimilation. Furthermore, they mentioned a subset of acetylation sites was deacetylase CobB regulated. To provide more details about the impact of deacetylase CobB on E. coli acetylome, serial dilution of SILAC-labeled peptides method was used for the accurate quantification of acetylation at 3,669 unique sites peptides (Weinert et al., 2017). The SILAC method 
provided a detailed view of acetylation stoichiometry confirming that deacetylase CobB suppresses acetylation. Another publication also determined the stoichiometry of lysine acetylation in E. coli. Chemical acetylation with isotopic acetic anhydride was used to quantify 2,206 peptides, and a wide distribution of acetyl stoichiometry from $1 \%$ up to $98 \%$ was observed (Baeza et al., 2014).

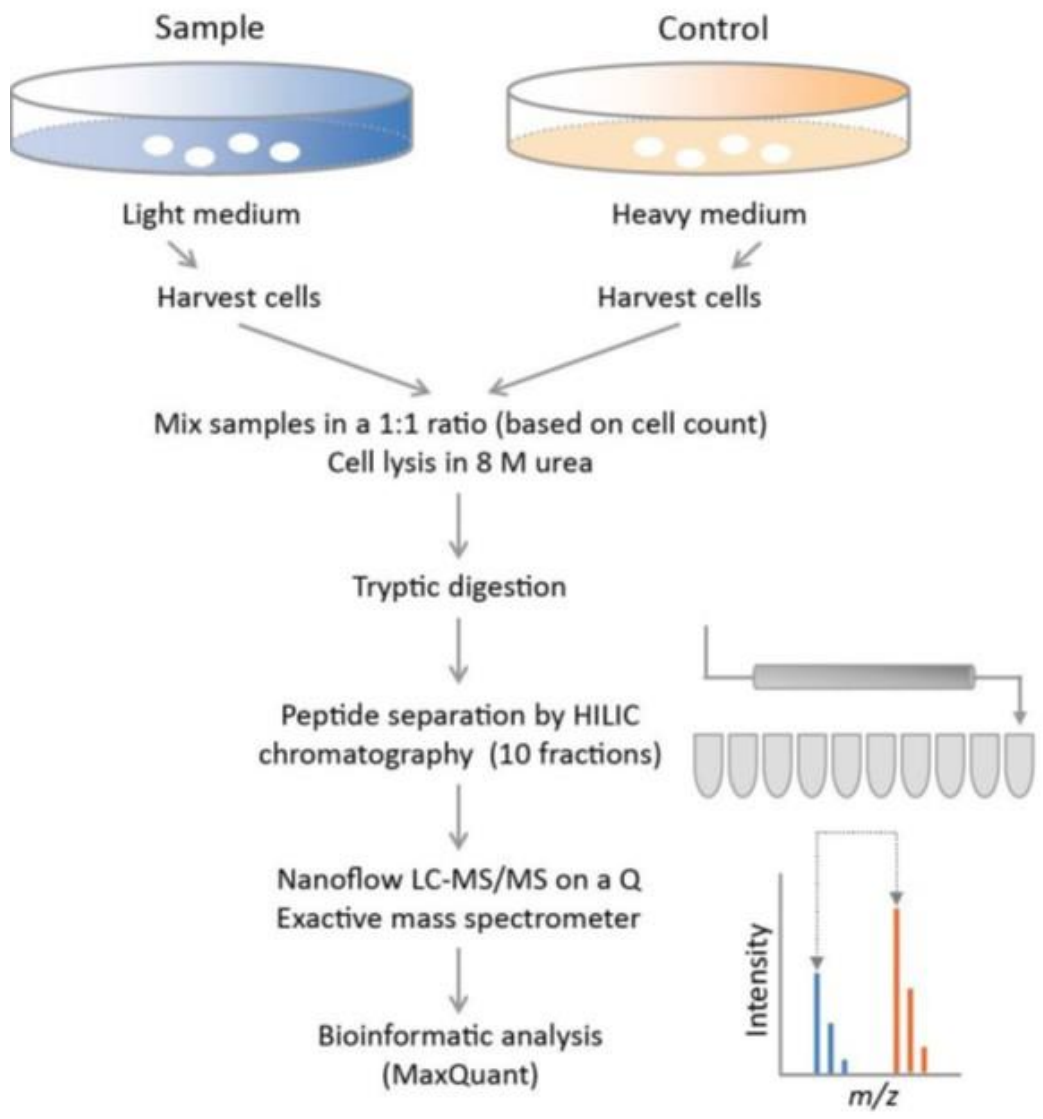

Figure 14 Workflow of SILAC (Demmers, 2015) 


\section{I.3.4 Functional consequences of bacterial protein acetylation}

Before the global proteomic studies of the bacterial acetylome, acetylation of acetyl-CoA synthetase (Starai et al., 2002) and chemotaxis response regulator CheY (Ramakrishnan et al., 1998) were shown to regulate acetate metabolism and chemotaxis. Acetyl-CoA synthetase (Acs), responsible for acetyl-CoA synthesis, is essential in acetate assimilation pathway (Wolfe, 2005). The activity of Acs in S. enterica is regulated by acetylation-deacetylation (Starai et al., 2002). Acetylation of the active lysine in Acs turned off the enzyme activity, while deacetylation by a $\mathrm{NAD}^{+}$-dependent sirtuin deacetylase CobB activated it. The inactivation effect by acetylation and the protection effect by sirtuin deacetylase exist in various bacteria species, including E. coli, B. subtilis, R.palustris, S. coelicolor, M. tuberculosis, M. smegmatis (Bernal et al., 2014). Interestingly, a similar event on Acs has been observed in mitochondria. In mitochondria, acetyl-CoA synthetases are feedback-regulated by inhibitory effect of GNAT acetylation and activating effect of sirtuins deacetylation (Hallows et al., 2006). Since then, more bacterial enzymes were found to be regulated by reversible acetylation. In E. coli, N-hydroxyarylamine Oacetyltransferase (NhoA) containing two acetylation sites can be deacetylated by CobB. Introducing mutation on lysine acetylation sites reduced NhoA activity (Zhang et al., 2013b).

As mentioned earlier, transcription factor p53 in eukaryotic cell is a target for PTM code. Such protein targeted by PTM code also exists in bacteria. Bourret and his colleague have investigated the role of acetylation of the response regulator CheY in E. coli (Ramakrishnan et al., 1998). Through their work and follow-up works on CheY acetylation, functions of $\mathrm{CheY}$ acetylation has been demonstrated that acetylation can fine-tune $\mathrm{CheY}$ 
associated chemotaxis by repressing the CheY binding and adjusting CheY activation (Barak and Eisenbach, 2001; Fraiberg et al., 2015; Liarzi et al., 2010; Yan et al., 2008). Acetylation, together with phosphorylation, provides dual levels of regulation on CheY interactions with CheY kinase CheA, CheY phosphatase CheZ and the switch protein FliM (Barak and Eisenbach, 2004; Liarzi et al., 2010). Acetylation also involved in clockwise flagellar rotation possibly due to the effect of acetylation on protein conformation (Fraiberg et al., 2015). Furthermore, acetylation cross-talks with other PTMs on CheY. The CheY acetylation is closely related and co-regulated with phosphorylation (Barak and Eisenbach, 2004; Li et al., 2013), and maybe also associated with methylation-demethylation in chemokinetic adaptation (Baron et al., 2017). The acetylation of CheY is either through nonenzymatic acetylation using $\mathrm{AcCoA}$ as the acetyl donor, or through enzymatic acetylation by Acs using AcCoA or acetate. The deacetylation of CheY is catalyzed by Acs or by deacetylase CobB (Barak et al., 2004).

It has been well established in eukaryotes that acetylation of histones and transcription factors regulates gene expression and other DNA centered processes. Similar cases are also present in bacteria. As mentioned, acetylation of the M. tuberculosis nucleoid associated protein $M t \mathrm{HU}$ by KAT Eis reduced DNA interaction ability and may even change genome structure (Ghosh et al., 2016). Acetylation of RNA polymerase (RNAP) is important for protein function. Acetylation on two lysine residues located on alpha subunit of RNAP regulated glucose-induced $c p x P$ transcription(Lima et al., 2011; Lima et al., 2012). Furthermore, acetylation of DNA replication initiator protein DnaA in E. coli 
inhibited ATP binding ability, which potentially affect DNA replication initiation(Zhang et al., 2016). Both RNAP and DnaA were deacetylated by CobB.

Acetylation also affect RNA metabolism. E. coli RNase R was acetylated by a KAT YfiQ during exponential phase. Acetylation stabilize the interaction of RNase R with tmRNA-SmpB complex, protecting RNase $\mathrm{R}$ from Lon protease degradation. While in stationary phase, RNase R is rapidly degraded because of the lack of acetylation (Liang and Deutscher, 2012; Liang et al., 2011). Acetylation of RNase II by YfiQ was found to associate with slow growth rate. The RNase II acetylation affected protein binding affinity thus reduced the protein activity (Song et al., 2016). Acetylation of RNase R and RNase II are reversed by CobB.

Compelling evidence revealed the significant roles of acetylation in the regulation of central metabolism. Proteomics studies revealed acetylation of a large set of metabolic enzymes in multiple bacterial species (Hentchel and Escalante-Semerena, 2015; Ouidir et al., 2016; Wolfe, 2016). Acetylated metabolic enzymes are involved in many pathways, including glycolysis, the tricarboxylic acid (TCA) cycle, fatty acid biosynthesis/degradation and the acetate kinase/ phosphotransacetylase pathway (Figure 15) 


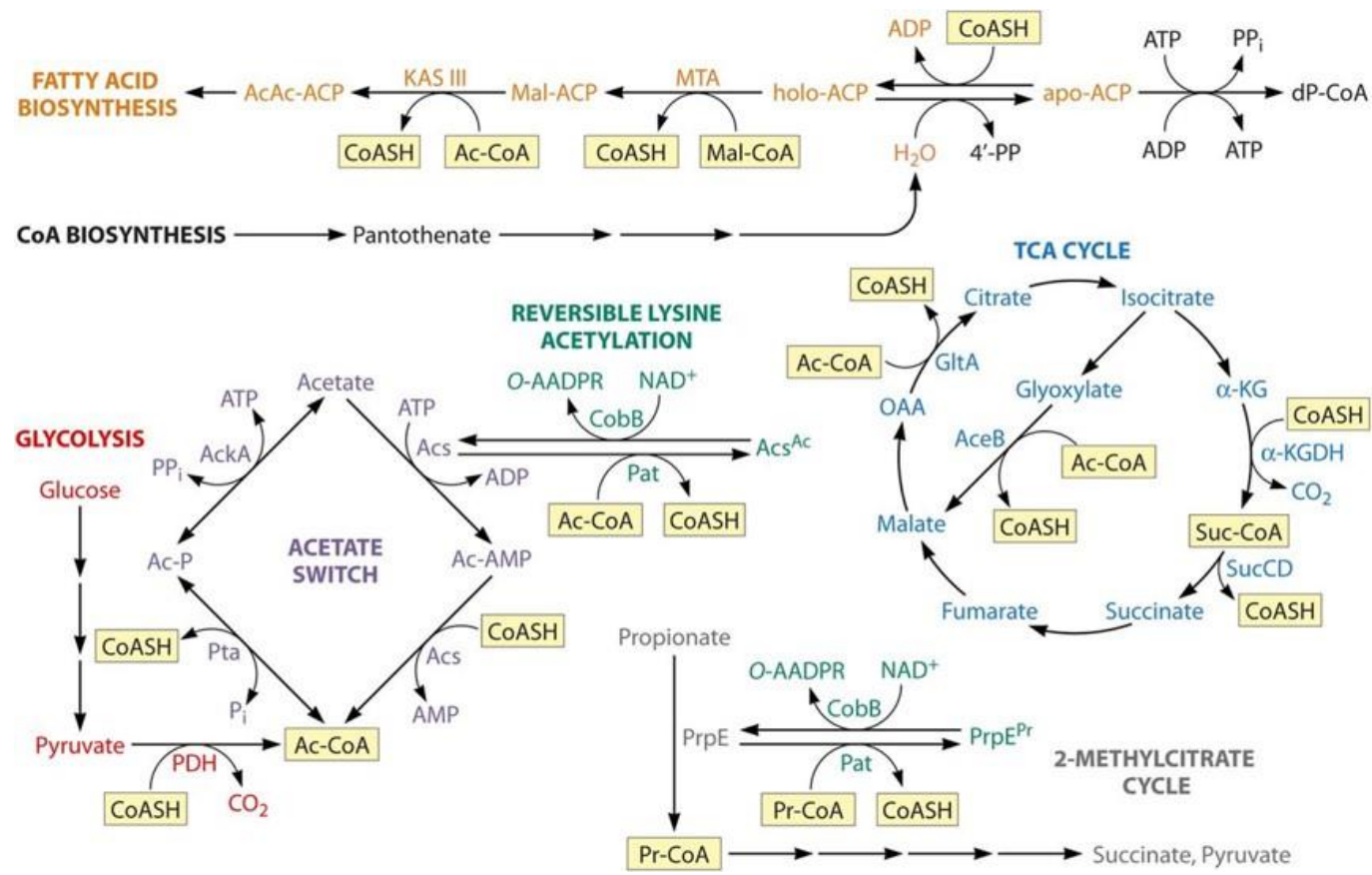

Figure 15 schematic of the contributions of $\mathrm{CoA}$ and acetyl-CoA to cellular metabolism (Hentchel and Escalante-Semerena, 2015)

Since exposure to different carbon sources alters acetylation on central metabolic enzymes, metabolic enzymes acetylation and carbon source utilization are tightly related. On one hand, acetylation may regulate carbon source utilization and metabolic flux. For instance, acetylation of $E$. coli proteins was accumulated in stationary phase. The growth phase dependent acetylation paralleled with glucose consumption and acetate excretion which began upon entry into stationary phase (Schilling et al., 2015). S. enterica metabolic enzymes were acetylated differentially in response to excess glucose or citrate (Wang et al., 2010). It seems that acetylation controls the direction of carbon utilization pathways under different conditions. On the other hand, acetylation depends on proper regulation of central metabolism. Bacterial acetyltransferases use acetyl-CoA as acetyl donor, linking 
acetylation with acetyl-CoA pools. Acetyl-CoA synthetase, which itself is subjected to acetylation, regulates acetyl-CoA level. Therefore, it seems that fluctuations in acetyl-CoA level affect acetylation, and the activity and stability of metabolic enzymes are regulated by acetylation, thus feedback regulate acetyl-CoA level (Wolfe, 2016).

Acetylation has been reported to play a role in stress response. Deletion of E. coli sirtuin deacetylase CobB increases resistance to heat stress and oxidative stress, but decreases acid stress resistance (Castano-Cerezo et al., 2014; Ma and Wood, 2011). Moreover, various stress-related genes are repressed by deacetylation, including genes related to heat shock, cold shock, osmotic stress and acid resistance, and carbon starvation (Ma and Wood, 2011).

\section{I.3.5 Enzymatic acetylation and acetyltransferases}

The conventional mechanism of lysine acetylation is the enzymatic acetylation, which is dependent on lysine acetyltransferases (KAT) to transfer the acetyl group to the $\varepsilon$-amino group of a lysine. Bacterial KATs belong to the Gcn5-related N-acetyltransferases (GNAT) superfamily conserved in all domains of life. The first bacterial acetyltransferase was discovered in Salmonella enterica in 2004 (Starai and Escalante-Semerena, 2004). The acetyltransferase, named $\mathrm{SePat}$, was able to carry out acylation reaction on acetyl-CoA synthetase and propiony-CoA synthetase. The SePat protein consists of two domains (Figure 16): an N-terminal NDP-forming AcCoA synthetase domain and a C-terminal GNAT RimL-acetyltransferase domain. RimL is a ribosomal protein acetyltransferase with a conserved GNAT domain (Miao et al., 2007). However, the NDP-forming AcCoA 
synthetase domain of $\mathrm{SePat}$ lacks the ability to produce AcCoA because the catalytic histidine residue in this domain is replaced (Thao and Escalante-Semerena, 2011a).

In E. coli, the only well-studied KAT is YfiQ, which is homologous of $\mathrm{SePat}$ characterized in S. enterica. It is possible that there are several other $E$. coli KATs yet to be identified in addition to YfiQ, with consideration of the facts that inactivation of KAT

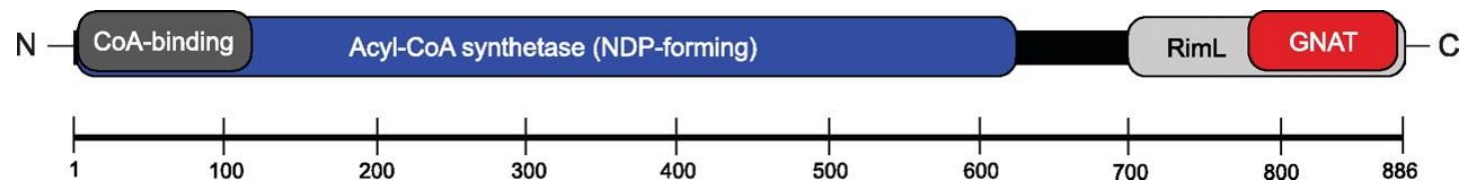

Figure 16 Domain structure of Salmonella enterica Pat (Thao and Escalante-Semerena, 2011a)

YfiQ in E. coli has very minor effect on global acetylation (Castano-Cerezo et al., 2014; Kuhn et al., 2014; Weinert et al., 2013). The minor effect of YfiQ inactivation rises the following possibilities: (i) there are other KATs in bacteria. The putative KATs could belong to GNAT family, or may belong to other novel families of KATs. (ii) some acylases may have dual functions to catalyze lysine acetylation reaction. For instance, $M$. tuberculosis Eis, a GNAT family aminoglycoside acetyltransferase, was discovered to acetylate nucleoid-associated protein $M t \mathrm{HU}$ (Ghosh et al., 2016). It is possible that other acylases may potentially catalyze lysine acetylation. (iii) lysine acetylation in bacteria is also carried out in a nonenzymatic process. A similar mechanism was found in mitochondria that mitochondrial proteins can be nonenzymatically acetylated by acetylCoA (Baeza et al., 2015; Pougovkina et al., 2014).

Currently, attempts to identify new KATs have not been successful. Studies of bacterial acylases for acetylation functions are currently not straight forward. New 
approaches should be developed for new KATs identification and functionality explorations of existing acylases. However, compelling evidence has demonstrated the existence of nonenzymatic acetylation in bacteria.

\section{I.3.6 Nonenzymatic acetylation}

In E. coli, protein acetylation can occur through the nonenzymatic process in addition to enzymatic process. The nonenzymatic acetylation is dependent on the direct acetyl group donation from acetyl-phosphate (acP) to the $\varepsilon$-amino group of a lysine. Acetyl phosphate is a high energy intermediate of phosphotransacetylase (Pta)-acetate kinase (AckA) pathway (Figure 17), which is also considered as a crucial signaling molecule (Fredericks et al., 2006; Wolfe et al., 2003). A large number of lysine acetylation sites in E. coli was found to be acetyl-phosphate regulated, while up to $10 \%$ of sites were regulated by YfiQ. Thus, it has been proposed that the global acetylation in E. coli was largely through nonenzymatic acetylation by acetyl-phosphate (Castano-Cerezo et al., 2014; Kuhn et al., 2014; Weinert et al., 2013).

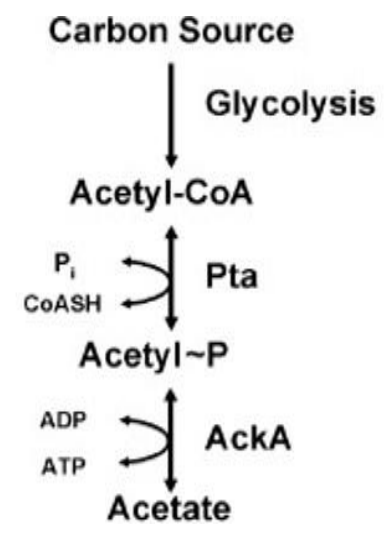

Figure 17 The phosphotransacetylase (Pta)-acetate kinase (AckA) pathway (Fredericks et al., 2006) 
Acetylation is likely to be affected by surrounding neighborhood. In diverse bacteria, acidic amino acids were enriched in the surrounding area of acetylation sites (Kim et al., 2013; Liao et al., 2014; Okanishi et al., 2013; Xie et al., 2015; Zhang et al., 2009a). The significant enrichment could be due to: (i) antibodies used for proteomic analysis of modified peptides are prone to recognize acidic amino acids enriched peptide sequences; (ii) these acidic amino acids enriched motifs may be a recognition signature for KATs; (iii) the surrounding acidic amino acids may be part of enzymatic and/or nonenzymatic acetylation reaction. These possibilities need further explorations. Determination of the spacing and functions of acidic amino acids residues would further expand the understanding of bacterial protein acetylation (Carabetta and Cristea, 2017).

\section{I.3.7 Deacetylases}

$\mathrm{N}^{\varepsilon}$-lysine acetylation is reversed by the protein deacetylase. As discussed above, two types of deacetylases are present in eukaryotes: Zinc (II)-dependent lysine deacetylase and $\mathrm{NAD}^{+}$-dependent sirtuin deacetylase. Both types of deacetylase are found in bacteria. The deacetylase CobB has been the best characterized sirtuin deacetylase in many bacteria species including E. coli, B. subtilis, S. enterica (Gardner and Escalante-Semerena, 2009; Starai et al., 2002; Zhao et al., 2004). The deacetylation function of sirtuin deacetylase CobB requires $\mathrm{NAD}^{+}$. Therefore, activities of sirtuinsare dependent on cellular $\mathrm{NAD}^{+}$level, which in turn reflect cell energy states (Hentchel and Escalante-Semerena, 2015). Compared to sirtuin which is broadly distributed in all three domains of life, Zinc (II) dependent lysine deacetylase is less frequent. LdaA in $R$. palustris (Crosby et al., 2010) 
and AcuC in B. subtilis (Gardner et al., 2006) belong to the zinc dependent lysine deacetylase.

In E. coli, sirtuin deacetylase CobB is the predominant lysine deacetylase showing no preference for enzymatically acetylated and non-enzymatically acetylated lysines. Not

all acetylated lysine sites are CobB-regulated suggesting the existence of other protein lysine deacetylases (AbouElfetouh et al., 2015). Recently, a novel deacetylase was discovered in E. coli. Deacetylase YcgC uses a serine residue as the catalytic nucleophile to remove acetyl groups from lysines and does not require $\mathrm{Zn}^{2+}$ or $\mathrm{NAD}^{+}$like other deacetylases. This novel family of deacetylase $\mathrm{YcgC}$ has distinct target substrates from CobB (Tu et al., 2015).

\section{OVERVIEW}

Escherichia coli topoisomerase I is essential in DNA supercoiling regulation. The relaxation action of topoisomerase I and supercoiling action of DNA gyrase maintain the balance of DNA supercoiling. E. coli topoisomerase I has been shown to be regulated by DNA supercoiling and the transcription of topA gene is associated with stress challenges. Previous proteomics studies have revealed that E. coli topoisomerase I is modified by reversible $\mathrm{N}^{\varepsilon}$-acetylation at multiple lysine residues suggesting the regulatory role of acetylation on topoisomerase I. In view of the significant role of TopA in regulating DNA supercoiling, studying the dynamic regulation of TopA by acetylation will help to understand its role in DNA metabolism and other cellular processes. 
This dissertation project explores the dynamic regulation of $E$. coli topoisomerase I via lysine acetylation, and further explores the physiological function of sirtuin deacetylase CobB in the regulation of topoisomerase I acetylation. Chapter one exploresthe effect of lysine acetylation on topoisomerase I. We showed that acetylation reduces topoisomerase I activity in both in vivo and in vitro assays. In Chapter two, the physiological significance of topoisomerase I acetylation has been examined. Topoisomerase I acetylation has global effects on DNA supercoiling and growth rate, the degree of acetylation is related to growth phases. Deacetylation of topoisomerase I by $E$. coli CobB prevents such effects from excess lysine acetylation. Chapter three studies the molecular mechanism underlying the topoisomerase I catalytic activity reduction by lysine acetylation. We predicted that the acetylation on certain lysine residues may be of importance. The lysine acetylation model of Lys-484 substitution suggested that acetylation on Lys-484 may contribute to the activity reduction. Our study revealed the first evidence for acetylation regulation of TopA catalytic activity and expression. It provides understanding of global effects on bacteria genome via $E$. coli topoisomerase I acetylation-deacetylation, and facilitates the functional study of deacetylase CobB which contributes to fully understand the role of acetylation-deacetylation in bacteria. 


\title{
CHAPTER ONE: ACETYLATION REDUCES E. COLI TOPIOSMERASE I \\ CATALYTIC ACTIVITY AND THE SIRTUIN DEACETYLASE COBB \\ PROTECTS IT
}

\begin{abstract}
Escherichia coli topoisomerase I (TopA), a regulator of global and local DNA supercoiling, is modified by $\mathrm{N}^{\varepsilon}$-Lysine acetylation. The $\mathrm{NAD}^{+}$-dependent sirtuindeacetylase CobB can reverse both enzymatic and non-enzymatic lysine acetylation modification in E. coli. Previous studies revealed the accumulated acetylation in $\triangle \operatorname{cobB}$ mutant. Here, we show that the absence of deacetylase CobB in a $\triangle c o b B$ mutant reduces intracellular TopA catalytic activity while elevating TopA expression and topA gene transcripts levels. The relaxation activity of purified TopA is decreased by in vitro non enzymatic acetyl phosphate mediated lysine acetylation. The interaction with purified CobB protects TopA from inactivation by such non-enzymatic acetylation. These findings demonstrate that $E$. coli TopA catalytic activity can be modulated by lysine acetylationdeacetylation, and the deacetylase $\mathrm{CobB}$ is important in the prevention of TopA inactivation from excess lysine acetylation.
\end{abstract}

\section{INTRODUCTION}

DNA supercoiling level is highly significant for vital cellular processes. Therefore, topoisomerases mainly responsible for DNA supercoiling regulation are required in every organism to prevent accumulation of excessive improper supercoiling (Chen et al., 2013). Hypernegative supercoiling has been demonstrated to cause RNA-DNA hybrid (R-loop) 
stabilization. Accumulation of R-loops inhibits DNA transcription and replication, leading to genomic instability (Drolet, 2006; Hamperl and Cimprich, 2014; Skourti-Stathaki and Proudfoot, 2014). Topoisomerase I encoded by the topA gene is an essential and ubiquitous enzyme present in eukaryote, bacteria and archaea. It binds to the single-stranded DNA region of a double helix and relaxes the negative supercoils. Topoisomerase I enzyme is required for preventing excess negative DNA supercoiling (Masse and Drolet, 1999b). Moreover, topoisomerase I has an important role in preventing R-loop accumulation since R-loop formation is caused by excess negative supercoiling (Masse and Drolet, 1999a). Extensive R-loop formation was found in the absence of topoisomerase I. The accumulation of R-loop as a result of the absence of topoisomerase I is more severe when RNAse $\mathrm{H}$ activity is depleted. The loss of topoisomerase I function may have additional consequences other than extensive R-loop formation, as overexpression of R-loop processing enzymes did not always suppress the lethality of topA deletion (Stockum et al., 2012). On the other hand, the transcription of topA gene is regulated by DNA supercoiling such that increasing of DNA negative supercoiling promotes topA transcription (Tse-Dinh, 1985). For convenience, E. coli topoisomerase I protein will be referred to TopA protein in the following context.

Post-translational modification of TopA could be an additional mechanism for regulation of TopA activity and DNA supercoiling. Post-translational modification is a reversible mechanism used by cells for rapid adaptation to changes in external environment without requiring the synthesis of new RNA and protein to fulfill the needs of adaptation to diverse growth conditions (Cain et al., 2014; Fan et al., 2015; Su et al., 2016). $\mathrm{N}^{\varepsilon}$-Lysine 
acetylation is one of the most abundant posttranslational modifications (Choudhary et al., 2009; Thao and Escalante-Semerena, 2011b). Recently, proteomic studies have demonstrated the broad existence of lysine acetylation not only in eukaryotes, but also in diverse bacterial species including E. coli (Drazic et al., 2016; Hu et al., 2010; Weinert et al., 2013; Yu et al., 2008; Zhang et al., 2009a). In addition, compelling evidence revealed the significant roles of $\mathrm{N}^{\varepsilon}$-Lysine acetylation in bacterial physiology for the regulation of central metabolism enzymes, motility, and chemotaxis gene expressions and stress resistance systems (Barak and Eisenbach, 2004; Bernal et al., 2014; Carabetta and Cristea, 2017; Castano-Cerezo et al., 2014; Wang et al., 2010). Two distinct mechanisms of lysine acetylation have been reported in E. coli (Wolfe, 2016). The first mechanism is enzymatic acetylation, which requires the $\mathrm{Pka} / \mathrm{YfiQ}$ enzyme, a Gcn5-like acetyltransferase, or other acetyltransferases yet to be identified, to transfer the acetyl group from acetyl-coenzyme A (acCoA) to the $\varepsilon$-amino group of a lysine (Thao and Escalante-Semerena, 2011b). The second mechanism is nonenzymatic acetylation, which is dependent on the direct acetyl group donation from acetyl-phosphate (acP) to the $\varepsilon$-amino group of a lysine (Kuhn et al., 2014; Weinert et al., 2013). Both enzymatically acetylated-lysine and nonenzymatically acetylated-lysine modifications are reversed by the $\mathrm{NAD}^{+}$- dependent sirtuin deacetylase CobB, a predominant lysine deacetylase in E. coli showing no preference for the two types of acetylated lysine sites (AbouElfetouh et al., 2015).

Previous proteomic studies of protein acetylation in E. coli demonstrated that multiple lysine residues of TopA were acetylated (Baeza et al., 2014; Kuhn et al., 2014; Weinert et al., 2013; Weinert et al., 2017; Zhang et al., 2009a; Zhang et al., 2013a). Lysine 
acetylation of TopA was found to be sensitive to acetyl phosphate (acP) levels (Kuhn et al., 2014; Weinert et al., 2013). Therefore, it appears that TopA lysine acetylation can occur through the nonenzymatic acetyl phosphate dependent mechanism. The NAD ${ }^{+}$ dependent sirtuin CobB deacetylase, the predominant deacetylase in E. coli, is reported to strongly interact with TopA (Liu et al., 2014). Given the frequency of observation of TopA acetylation and its interaction with CobB, acetylation-deacetylation may have an impact on TopA function as well as other facets of cell physiology that can be influenced by DNA supercoiling.

We proposed that acetylation on E. coli TopA reduces the enzyme catalytic activity, consequently affecting cell physiology. To test this hypothesis, we utilized a $\triangle \operatorname{cobB} E$. coli strain JW1106 from the Keio Collection and the parent E. coli strain BW25113 to evaluate the in vivo effects of TopA acetylation on cellular TopA catalytic activity, TopA protein expression and topA transcription response. The direct effect of non-enzymatic acPdependent lysine acetylation on TopA catalytic activity and the counter effect of purified CobB lysine deacetylase on TopA catalytic activity were demonstrated with in vitro biochemical experiments.

\section{MATERIALS AND METHODS}

\section{Materials}

The E. coli strains BW25113 [4(araD-araB)567 AlacZ4787(::rrnB-3) $\lambda^{-}$rph-1 $\Delta\left(\right.$ rhaD-rhaB)568 hsdR514], JW1106-1 [4(araD-araB)567 AlacZ4787(::rrnB-3) $\lambda^{-}$ AcobB 779::kan rph-1 $4($ rhaD-rhaB)568 hsdR514] were provided by the Yale Coli Genetic Stock Center. pETOP plasmid is a derivative of $\mathrm{pBAD} /$ Thio (Invitrogen) expressing 
recombinant $E$. coli TopA (Cheng et al., 2005). The ASKA plasmid pCA24N that encodes CobB with an N-terminal His 6 -tag was provided by NBRP (NIG, Japan): E. coli (Kitagawa et al., 2005). The E. coli cobB gene was amplified using the primers: 5'TGACGATGACAAGCTCGCCCTTATGGA AAAACCAAGAGTACTCGTACTG-3', and 5'-GGGATAGGCTTACCTTCAAGCTCGCCG GCATGCTTCCCGCTT TTAATC3' and ASKA plasmid pCA24NCobB as the template with Q5 High-fidelity DNA polymerase (New England Biolabs). The PCR product was cloned into pBAD/Thio to create plasmid pCobB using HiFi DNA assembly master mix (New England Biolabs). Cells were cultured in Lysogeny broth (LB) with shaking at $200 \mathrm{rpm}$ at $37^{\circ} \mathrm{C}$. When required, antibiotics were added at the following final concentrations: carbenicillin $50 \mu \mathrm{g} / \mathrm{ml}$, kanamycin $50 \mu \mathrm{g} / \mathrm{ml}$.

\section{Assay of TopA relaxation activity in cell lysates}

Wild type and $\triangle \operatorname{cobB}$ mutant strains were grown in $\mathrm{LB}$ at $37^{\circ} \mathrm{C}$ with shaking until $\mathrm{OD}_{600}=0.8$. Cells were pelleted by centrifugation at $4^{\circ} \mathrm{C}$ and lysed by lysozyme treatment in lysis buffer $\left(50 \mathrm{mM} \mathrm{NaH}_{2} \mathrm{PO}_{4}, 0.3 \mathrm{M} \mathrm{NaCl}\right)$ on ice and three freeze-thaw cycles. The total soluble lysates were obtained as the supernatant fractions following centrifugation at $30,000 \mathrm{rpm}$ at $4^{\circ} \mathrm{C}$ for $1 \mathrm{~h}$. Protein concentrations were determined using the Bradford Protein Assay (Bio-Rad). TopA activity in cell lysates was assayed in a standard reaction volume of $20 \mu \mathrm{l}(10 \mathrm{mM}$ Tris- $\mathrm{HCl}$, pH 8.0, $50 \mathrm{mM} \mathrm{NaCl}, 0.1 \mathrm{mg} / \mathrm{ml}$ gelatin, $6 \mathrm{mM} \mathrm{MgCl} 2$, 150 ng of supercoiled pBAD/Thio plasmid DNA). Purified recombinant TopA was added to relax the DNA substrate in control reactions. After 20 min incubation at $37^{\circ} \mathrm{C}$, the reaction was stopped by addition of $4 \mu 1$ stop solution (50 mM EDTA, 50\% glycerol, $0.5 \%$ 
(v/v) bromopheno blue). The DNA was electrophoresed in $0.8 \%$ agarose gel with TAE buffer containing $1 \mu \mathrm{g} / \mathrm{ml}$ chloroquine. To remove chloroquine, the gel was washed by shaking in TAE buffer for $2 \mathrm{~h}$ with the buffer replaced every $30 \mathrm{~min}$. After the removal of chloroquine, the gel was stained with ethidium bromide and visualized with UV light.

\section{Western blot analysis of TopA protein in cell lysates}

Total cell lysates containing $20 \mu \mathrm{g}$ of soluble proteins in SDS gel sample buffer $(2 \%$ SDS, $62.5 \mathrm{mM}$ Tris- $\mathrm{HCl}, \mathrm{pH} 6.8$ ) was heated at $100^{\circ} \mathrm{C}$ for $5 \mathrm{~min}$. After separation by electrophoresis in 10\% SDS-polyacrylamide gel, the total proteins in the gel was subjected to western blot analysis with mouse monoclonal antibody against $E$. coli TopA described previously (Stewart et al., 2005). Signals were developed with ECL Plus reagents (Thermo Scientific), and detected with C-DiGit Blot Scanner (LI-COR). Bands were quantified using the Image Studio Digits Ver 4.0 software. P-value was calculated by one-tailed student $t$ test.

\section{Quantitative polymerase chain reaction of topA mRNA}

Wild type and $\triangle c o b B$ cells were grown to $\mathrm{OD}_{600}$ of 0.8 . Total RNA was isolated using the Quick-RNA mini prep Kit (Zymo Research), and digested by DNase I (New England BioLabs). The cDNA template was synthesized in $20 \mu 1$ reaction from $1 \mu \mathrm{g}$ RNA using the iScript cDNA Synthesis Kit (Bio-Rad) and the following reaction conditions: $25^{\circ} \mathrm{C}$ for $5 \mathrm{~min} ; 42^{\circ} \mathrm{C}$ for $30 \mathrm{~min} ; 85^{\circ} \mathrm{C}$ for $5 \mathrm{~min}$. RNA and cDNA concentrations were measured using the BioPhotometer Plus (Eppendorf). The quantitative real-time polymerase chain reaction (qPCR) products were obtained using the following primers (Table 2). The relative level of topA and internal reference $i d n T, h c a T$ transcripts was 
quantified in $10 \mu \mathrm{l}$ reaction (10 ng cDNA, $0.25 \mu \mathrm{M}$ primers, $1 \mathrm{X}$ SYBR Green supermix) with the RT-PCR detection system CFX96 (Bio-Rad). PCR amplification was carried out in triplicate using the following cycling conditions: $95^{\circ} \mathrm{C}$ for $30 \mathrm{~s}$; followed by 40 cycles of $95^{\circ} \mathrm{C}$ for $20 \mathrm{~s} ; 60^{\circ} \mathrm{C}$ for $20 \mathrm{~s} ; 72^{\circ} \mathrm{C}$ for $30 \mathrm{~s}$. The melting curve analysis was performed after qPCR reaction with the temperature set to rise from $65^{\circ} \mathrm{C}$ to $95^{\circ} \mathrm{C}$. The topA expression level was normalized to $i d n T$ or $h c a T$ expression level as the internal reference. Results obtained were analyzed with the CFX Manager Software (version 3.1, Bio-Rad). $\mathrm{P}$-value was calculated by one-tailed student $\mathrm{t}$ test.

\begin{tabular}{|l|l|}
\hline Primer names & Primer sequences (5'-3') \\
\hline topA forward & 5'-ATCTGCCGGAAAGTCCGAATCAGT-3' \\
\hline topA reverse & 5'-TCTGCGCATCTGCTTCCATATCCT-3' \\
\hline idnT forward & 5'-CATCTGTTTAGCGAAGAGGAGATGC-3' \\
\hline$i d n T$ reverse & 5'-ACAAACGGCGGCGATAGC-3' \\
\hline hcaT forward & 5'-GCTGCTCGGCTTTCTCATCC-3' \\
\hline hcaT reverse & 5'-CCAACCACGCAGACCAACC-3' \\
\hline
\end{tabular}

Table 2 Primer sequences used in qPCR analysis

\section{Expression and Purification of recombinant E. coli TopA and CobB}

Recombinant TopA was expressed and purified from LB cultures of E. coli BL21AI (Invitrogen) transformant of TopA expression clone as described previously (Narula et al., 2011). Recombinant CobB with $\mathrm{N}$-terminal polyhistidine tag was expressed from the ASKA clone pCA24NCobB in BL21-AI by induction at $24^{\circ} \mathrm{C}$ with addition of $0.02 \%$ arabinose and 1mM Isopropyl $\beta$-D-1-thiogalactopyranoside (IPTG), followed by overnight 
growth at $24^{\circ} \mathrm{C}$. The CobB protein in the soluble lysates of the induced culture was purified using a Ni Sepharose column (GE) according to published procedures (Kitagawa et al., 2005).

\section{In vitro non-enzymatic lysine acetylation and CobB mediated deacetylation reactions}

In the in vitro nonenzymatic acetylation reaction, $1 \mu \mathrm{g}$ of $E$. coli TopA was incubated with $2 \mathrm{mM}$ and $5 \mathrm{mM}$ acP at $37^{\circ} \mathrm{C}$ in $150 \mathrm{mM}$ Tris- $\mathrm{HCl}(\mathrm{pH} 8.0), 10 \%$ glycerol, and $10 \mathrm{mM} \mathrm{MgCl} 2$ for 4 hours. The acetylation reaction was stopped by adding an equal volume of 2X SDS loading buffer and then boiling for $10 \mathrm{~min}$ before SDS PAGE and western blot analysis of lysine acetylation using mouse monoclonal anti-acetyllysine antibody (Cell Signaling Technology). The TopA protein on the membrane was then stained with Coomassie blue. For simultaneous in vitro acetylation/ deacetylation, $1 \mu \mathrm{g}$ of E. coli TopA was incubated with $0.2 \mu \mathrm{g}$ CobB in $150 \mathrm{mM}$ Tris- $\mathrm{HCl}(\mathrm{pH} 8.0), 10 \mathrm{mM}$ $\mathrm{MgCl}_{2}, 5 \mathrm{mM}$ acP, $0.25 \mathrm{mM} \mathrm{NAD}^{+}$, and $10 \%$ glycerol for 4 hours at $37^{\circ} \mathrm{C}$. To determine the effect of acetylation on TopA catalytic activity, $2 \mu 1$ of the acetylation /deacetylation reaction was used for serial dilutions and assay of TopA relaxation activity.

\section{Pull-down assays to study direct interaction between E. coli CobB and TopA}

Purified recombinant TopA protein $(5 \mathrm{nM})$ was mixed with purified $\mathrm{N}$-terminal His-tagged CobB protein (10-50 nM) in pull-down buffer (10 mM HEPES, pH 7.4, 100 $\mathrm{mM} \mathrm{NaCl}, 0.005 \% \mathrm{v} / \mathrm{v}$ Tween-20) overnight at $4^{\circ} \mathrm{C}$. HisPur Cobalt Agarose resin (Thermo Fisher), pre-equilibrated in pull-down buffer, was then added to the CobB-TopA reaction and mixed at $4{ }^{\circ} \mathrm{C}$ for $2 \mathrm{~h}$. After centrifugation of the reactions, the cobalt agarose resin was 
washed three times in pull-down buffer with $10 \mathrm{mM}$ imidazole. The proteins bound to the resin were eluted in SDS sample buffer by boiling for $2 \mathrm{~min}$. Eluates were electrophoresed in $10 \%$ polyacrylamide SDS gel, and TopA was detected by western blotting with monoclonal antibodies against E. coli TopA.

\section{RESULTS}

\section{Loss of CobB reduces cellular TopA activity while TopA expression is increased}

We hypothesized that the increased lysine acetylation on TopA in the $\triangle \operatorname{cob} B$ mutant cell would lead to reduction in TopA catalytic activity. TopA catalytic activity in total cell lysate of the wild-type BW25113 and the $\triangle \operatorname{cobB}$ mutant JW1106 was compared in assay with negatively supercoiled plasmid DNA as the substrate. The result showed that TopA from the total lysate of the $\triangle c o b B$ mutant displayed reduced catalytic activity (Figure 18, top panel). The reaction buffer used in the Figure 18 experiments did not contain ATP so gyrase and topoisomerase IV present in the cell lysates would not be active on the plasmid DNA substrate under these experimental conditions. Complementation by plasmid pCobB restored the TopA relaxation activity in the $\Delta \operatorname{cobB}$ mutant cell lysate (Figure 18, bottom panel). Overexpression of CobB in the wild-type $c o b B$ background did not result in higher relaxation activity. These results suggest that lack of normal physiological levels of CobB deacetylation activity could result in reduction of TopA catalytic activity. 

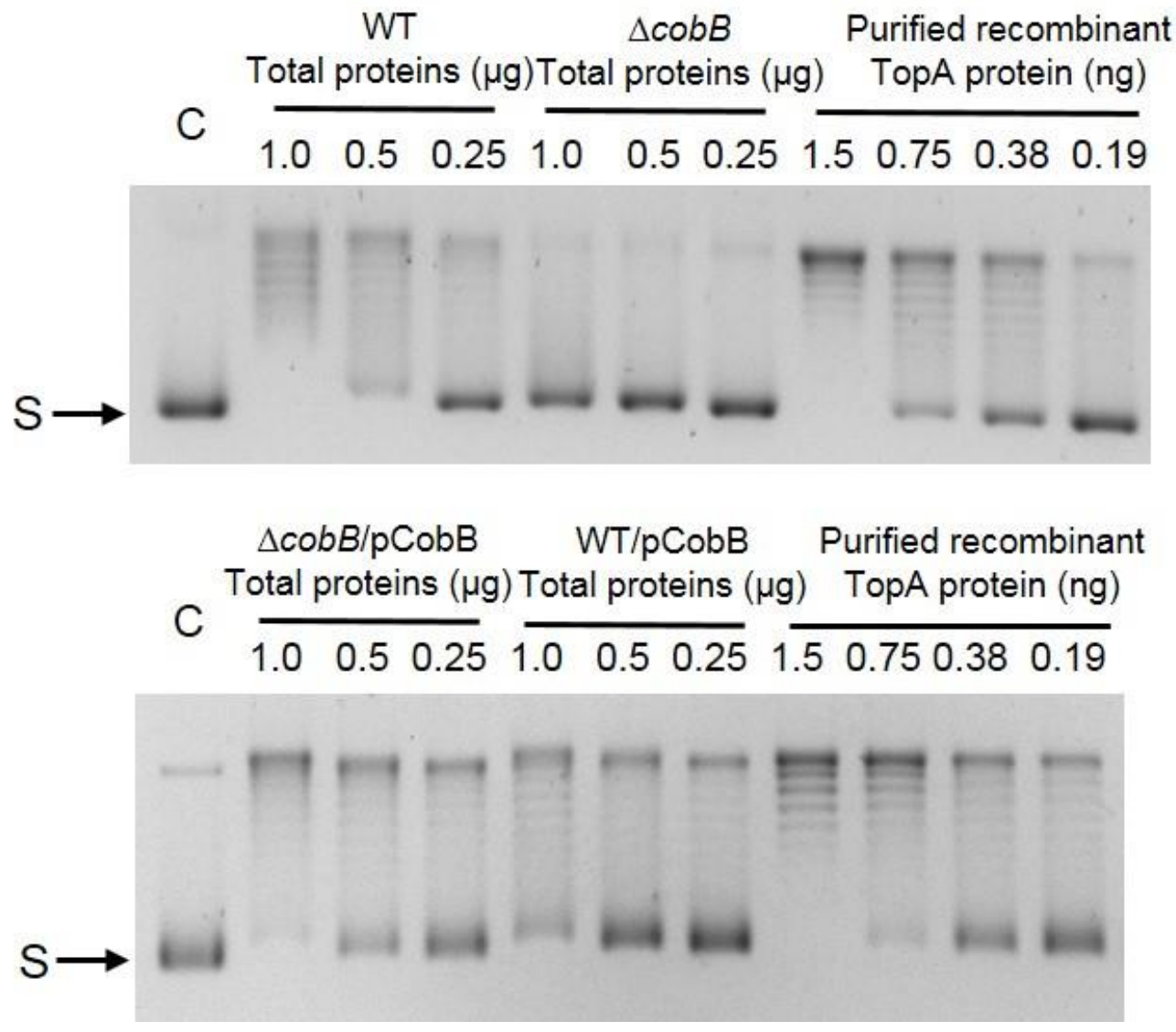

Figure 18 Relaxation assay of TopA activity in total soluble cell lysates of BW25113 (wild-type) and JW1106 ( $\triangle \operatorname{cobB})$ and their pCobB transformants (Zhou et al., 2017)

The indicated amount of total soluble proteins from BW25113 (wild-type) and JW1106 ( $\triangle$ cobB) cultures at $\mathrm{OD}_{600}=0.8$ were incubated with $150 \mathrm{ng}$ of negatively supercoiled plasmid DNA substrate $(\mathrm{S})$ at $37^{\circ} \mathrm{C}$ for $20 \mathrm{~min}$. DNA were electrophoresed in $0.8 \%$ agarose gel with TAE buffer containing $1 \mu \mathrm{g} / \mathrm{ml}$ chloroquine at $3 \mathrm{~V} / \mathrm{cm}$ for $16 \mathrm{~h}$. Reaction products from partial relaxation of the supercoiled plasmid DNA substrate by increasing amounts of purified recombinant TopA are included to show the effect of increasing TopA catalytic activity on plasmid electrophoretic mobility. 
However, an alternative possibility of the reduction of TopA activity in the $\Delta c o b B$ mutant is that the intracellular TopA protein level in the mutant strain is lower, resulting in reduced TopA activity in total cell lysate. To determine this possibility, TopA protein level and topA mRNA level were analyzed. TopA protein level in wild-type and $\Delta \operatorname{cobB}$ mutant cells was measured by western blot analysis. Total soluble lysates obtained from wild type and $\triangle \operatorname{cob} B$ cells were processed in parallel and the same amount of total proteins were loaded onto an SDS-PAGE gel. To compare the relative abundance of TopA from the same amount of total protein, equal loading of total cellular proteins based on Bradford protein assay results was confirmed by Coomassie blue staining (Figure 19). Western blot analysis showed that TopA protein level was significantly increased in the $\triangle \operatorname{cobB} B$ mutant, approximately 1.6-fold higher than that in wild-type cells with $\mathrm{P}$ value $\leq 0.05$ ( $\mathrm{P}$ value $=$ 0.016) (Figure $20 \mathrm{~A}, \mathrm{~B}$ ). Measurement of topA mRNA level by quantitative PCR also confirmed the increase in topA transcripts in the $\triangle \operatorname{cobB}$ mutant, about 1.4-fold to 1.5-fold increase with $h c a T$ and $i d n T$ as internal references (Figure 21). P value with $h c a T$ reference gene is 0.00019 , with $i d n T$ reference gene is 0.0019. $h c a T$ and $i d n T$ are validated reference gene invariant across different growth conditions (Peng et al., 2014; Zhou et al., 2011). These results showed the reduction in the TopA catalytic activity in the $\Delta c o b B$ cell extract (Figure 18) was the result of a decrease in the TopA protein level. However, even with the increased TopA expression, there is a deficiency in the TopA catalytic activity in the $\triangle \operatorname{cob} B$ mutant. 


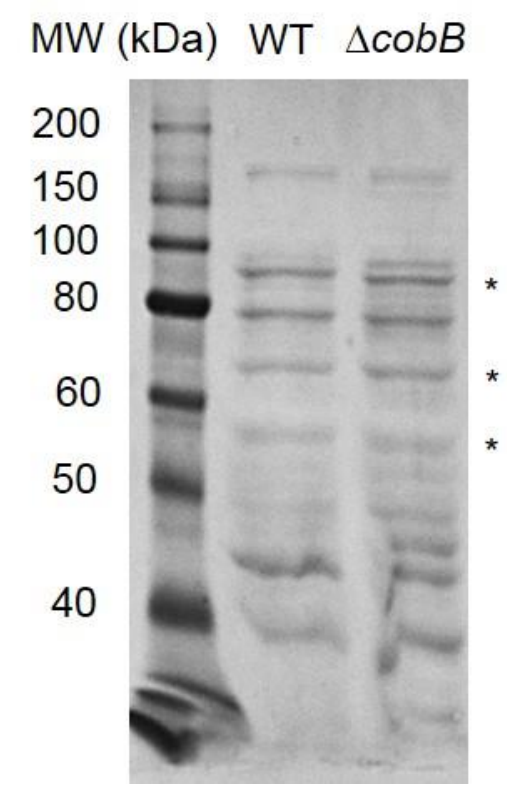

Figure 19 Coomassie blue staining of total cellular proteins in cell extract of wild-type BW25113 (WT) and $\Delta \operatorname{cobB}$ mutant JW1106 (Zhou et al., 2017)

Densitometry analysis of three bands $\left(^{*}\right)$ showed an average of $8 \%$ difference in intensities to confirm equal loading of total cellular proteins. MW: molecular weight standards. 

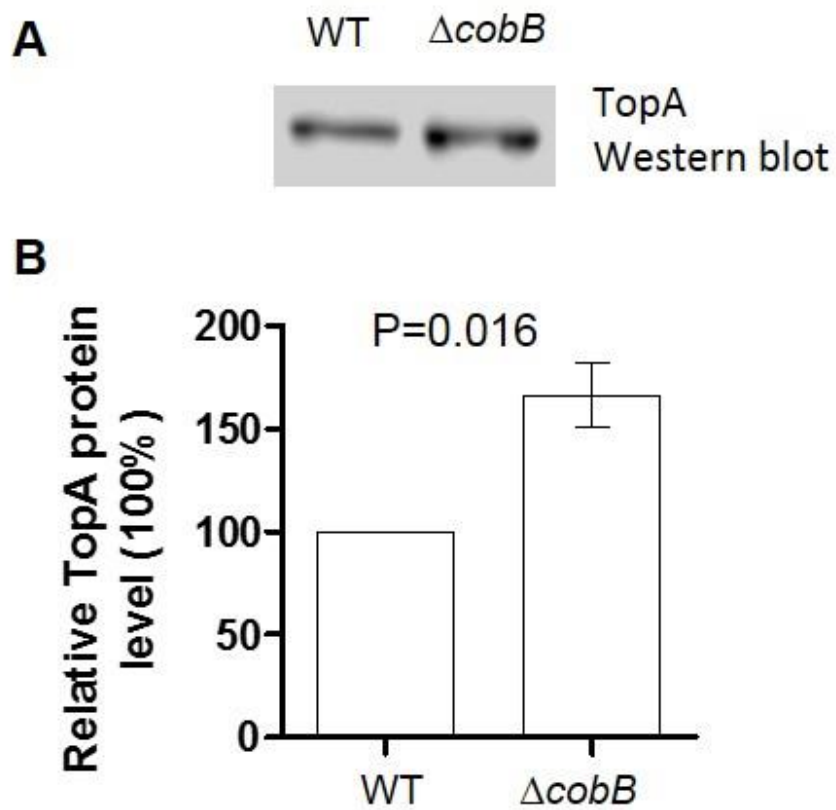

Figure 20 TopA expression level in BW25113 (wild-type) and JW1106 ( $\triangle$ cobB) strains (Zhou et al., 2017)

(A) $20 \mu \mathrm{g}$ total cell lysate was resolved by $10 \%$ SDS-PAGE gel, TopA protein expression level was detected by western blot using mouse monoclonal anti TopA antibody. (B) Quantification of four independent experiments as shown in (A). TopA expression in wild-type strain was set as $100 \%$, the relative TopA protein level in $\triangle \operatorname{cobB}$ mutant strain is shown. Error bars indicate standard deviation of relative TopA expression level in the $\triangle \operatorname{cobB} B$ mutant strain. 

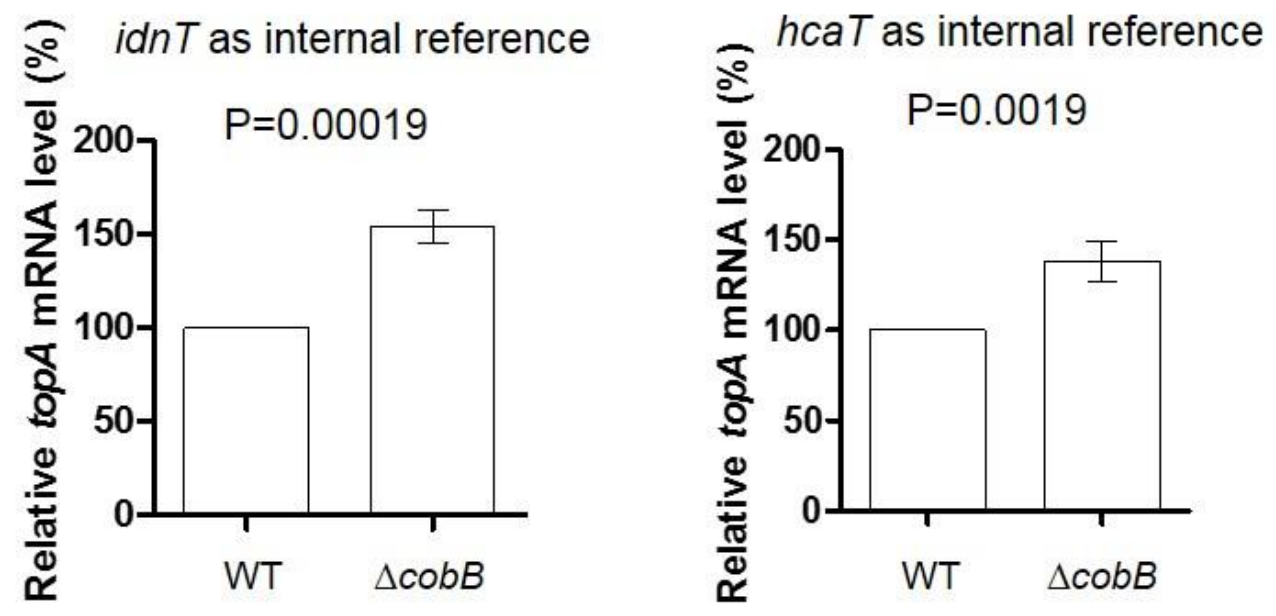

Figure 21 TopA transcripts level in BW25113 (wild-type) and JW1106 (AcobB) strains (Zhou et al., 2017)

Quantification of qPCR measurements of topA transcripts level from three individual experiments normalized against either $i d n T$ or $h c a T$ as internal references. topA transcripts level in wild-type strain was set as $100 \%$, the relative topA transcripts level in $\triangle \operatorname{cobB}$ mutant strain is shown. Error bars indicate standard deviation relative topA transcripts level in $\triangle \operatorname{cobB}$ mutant strain. 


\section{Acetylation by acP decreases TopA catalytic activity}

To examine the effect of lysine acetylation on TopA catalytic activity directly, we performed in vitro non-enzymatic lysine acetylation of TopA. Recombinant TopA expressed in a $c o b B+$ genetic background, was purified and incubated with acetyl phosphate (acP). According to previous study, the intracellular concentration of acP in $E$. coli reaches at least $3 \mathrm{mM}$, and may be as high as $4.5 \mathrm{mM}$ in wild-type cells (Klein et al., 2007). In the in vitro acetylation assay, purified TopA was incubated with $2 \mathrm{mM}$ and $5 \mathrm{mM}$ acP. Increase in TopA lysine acetylation level in the presence of $2 \mathrm{mM}$ and $5 \mathrm{mM}$ acP was observed by western blotting with antibodies against acetyl-lysine (Figure 22A). Assays with negatively supercoiled plasmid DNA as the substrate showed that the relaxation activity of TopA was reduced by approximately 4-fold following acetylation by $2 \mathrm{mM}$ acP and more than 8 -fold following acetylation by $5 \mathrm{mM}$ acP (Figure $22 \mathrm{~B}$ ). The in vitro acetyl phosphate mediated TopA acetylation assay clearly confirmed our hypothesis that lysine acetylation directly reduces TopA catalytic activity.

\section{Lysine deacetylation by CobB can counter the effect of non-enzymatic lysine acetylation on TopA catalytic activity}

Lysine acetylation is a reversible post-translational modification. In a living cell, acetylation-deacetylation can take place simultaneously at the same lysine residues. We showed evidence that TopA relaxation activity in $\triangle \operatorname{cobB}$ cell lysate is reduced, and nonenzymatic acetylation of purified TopA resulted in a decrease of its catalytic activity. Hence, the effect of CobB lysine deacetylase on TopA inactivation by acetyl phosphate mediated acetylation was examined next. TopA was incubated with both $2 \mathrm{mM}$ acP and 
purified CobB, so that acetylation and deacetylation can both take place. Notably, the acetylation level of TopA was reduced by CobB (Figure 23A). Accordingly, as shown in Figure 23B, the presence of CobB partially protected TopA catalytic activity from the inhibitory effect of acP-dependent lysine acetylation. These results indicate that CobB is important for maintaining TopA deacetylation and TopA catalytic activity. The partial protection by CobB suggests that other deacetylases may be involved in TopA deacetylation.

A

\begin{tabular}{|c|c|c|}
\hline 0 & 25 & $\mathrm{AcP}(\mathrm{mM})$ \\
\hline & U D & AcLys Western Blot \\
\hline & 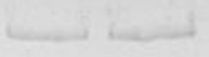 & Coomassie Staining \\
\hline
\end{tabular}

B

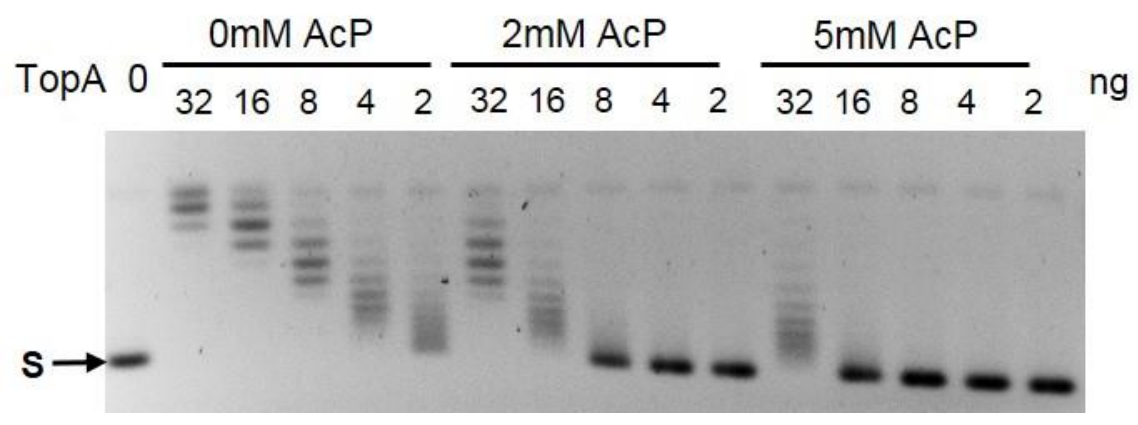

Figure 22 Acetyl phosphate mediated nonenzymatic lysine acetylation results in decrease in TopA catalytic activity (Zhou et al., 2017).

(A) Western blot analysis of acP acetylated TopA. Purified TopA $(1 \mu \mathrm{g})$ was incubated with $2 \mathrm{mM}$ and $5 \mathrm{mM}$ acP at $37^{\circ} \mathrm{C}$ for $4 \mathrm{hrs}$. The acetylation level of TopA was detected by western blot analysis using anti acetyllysine antibody. TopA on the membrane was stained with Coomassie blue staining. (B) Following incubation with and without acetyl phosphate, serial dilution of TopA (32ng, 16ng, 8ng, 4ng, $2 \mathrm{ng}$ ) was incubated with $150 \mathrm{ng}$ supercoiled plasmid DNA (S) at $37^{\circ} \mathrm{C}$ for $30 \mathrm{~min}$ to assay TopA catalytic activity. 


\section{$\mathrm{A} \quad-\quad-+\quad+\mathrm{AcP}$ \\ -+-+ CobB \\ AcLys Western Blot \\ Coomassie Staining}

B

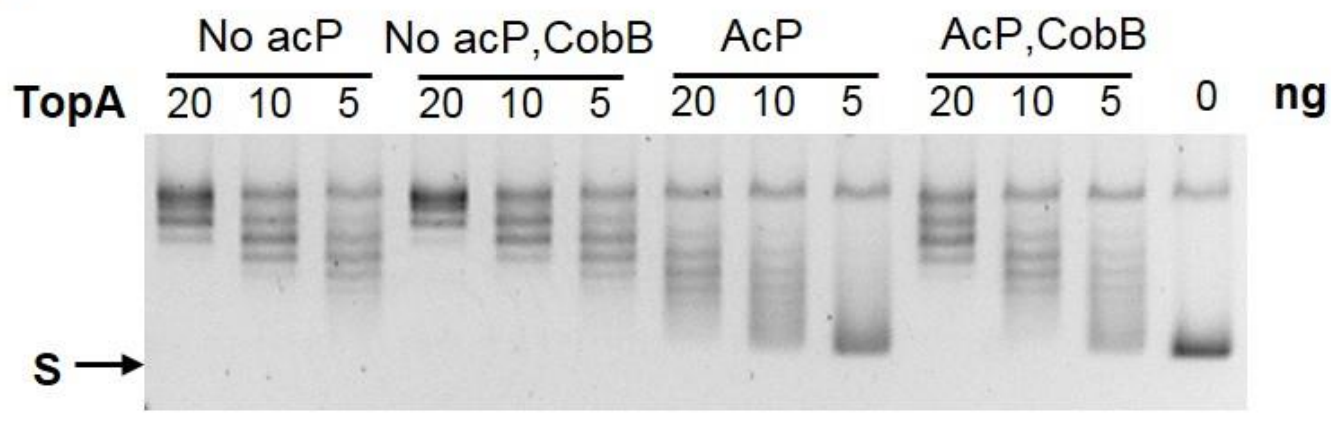

Figure 23 CobB deacetylation counters the effect of acetyl phosphate mediated lysine acetylation on TopA relaxation activity (Zhou et al., 2017).

Purified E. coli TopA $(1 \mu \mathrm{g})$ and $2 \mathrm{mM}$ acetyl phosphate (acP) was incubated with or without CobB $\left(0.65: 1 \mathrm{molar}\right.$ ratio to TopA) at $37^{\circ} \mathrm{C}$ for $4 \mathrm{hrs}$. (A) Acetylation level of TopA was compared by western blot analysis with anti-acetyl lysine antibody. TopA protein on the nitrocellulose membrane was visualized by Coomassie blue staining. (B) Following incubation with acP in the absence or presence of CobB, serial dilutions of TopA (20 ng, $10 \mathrm{ng}, 5 \mathrm{ng}$ ) were incubated with $150 \mathrm{ng}$ negatively supercoiled plasmid DNA (S) at $37^{\circ} \mathrm{C}$ for $30 \mathrm{~min}$ to assay the TopA relaxation activity. 


\section{TopA interacts directly with CobB in the absence of DNA}

TopA has been demonstrated in a previous study to be a CobB binding protein using a proteome microarray as well as bio-layer interferometry analysis (Liu et at.,2014). To confirm that TopA interacts directly with CobB in the absence of DNA, purified recombinant TopA and CobB proteins were mixed in binding buffer. HisPur Cobalt resin was used to pulldown TopA in complex with the N-terminal His-tagged CobB. Western blot analysis of the proteins bound to the Cobalt resin with antibodies against TopA (Figure 24A) showed that the presence of His-tagged CobB allows binding of TopA to the Cobalt resin and co-elution with the His-CobB (Figure 24B, C). The direct interaction of TopA and CobB further confirmed that the deacetylation reaction of TopA is mediated by CobB through TopA and CobB interaction in the absence of DNA. 


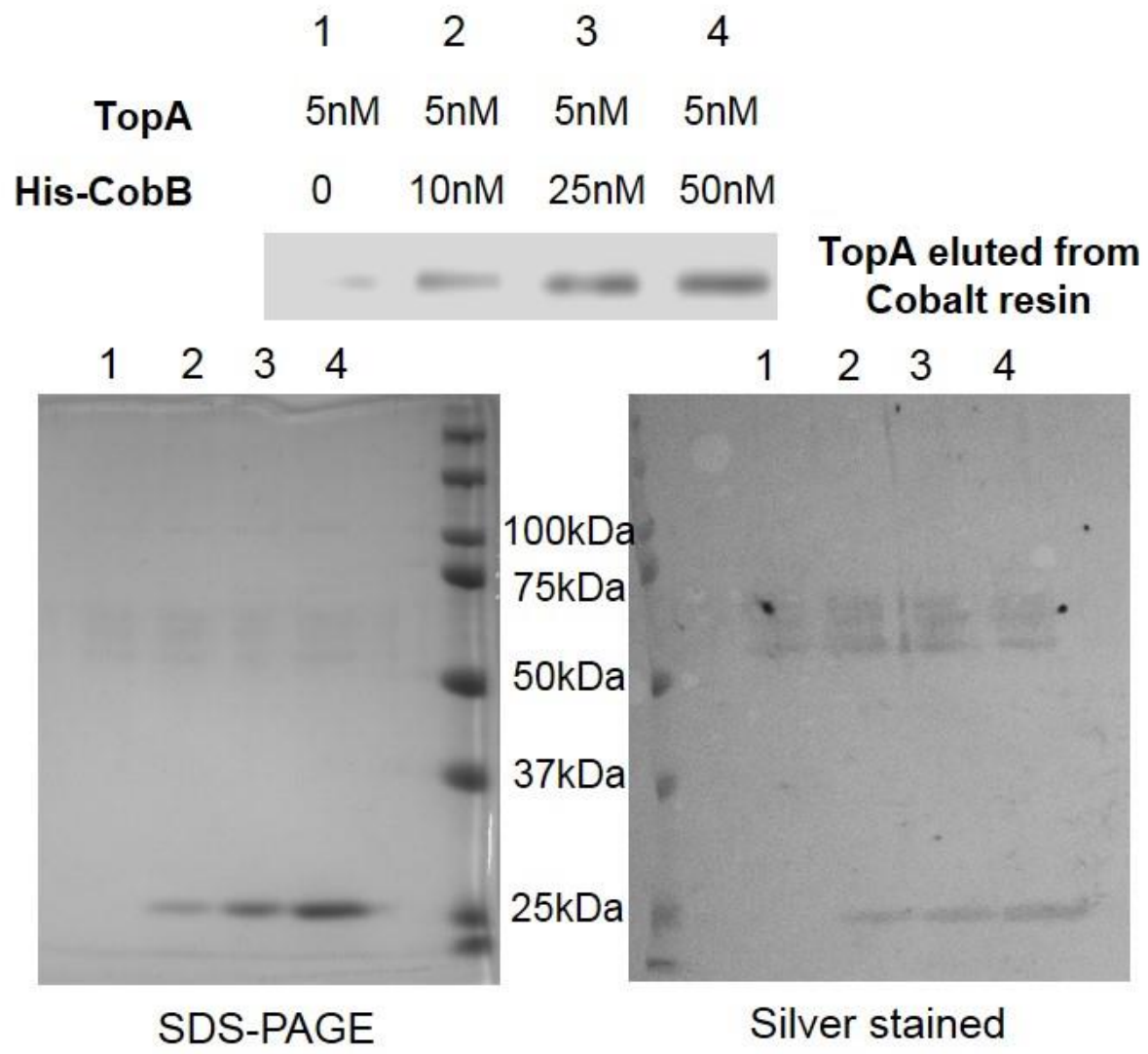

Figure 24 Direct interaction between TopA and CobB (Zhou et al., 2017).

Purified recombinant TopA and His-tagged CobB were allowed to interact before pull-down by HisPur Cobalt Agarose Resin. The presence of TopA in the bound proteins eluted from the Cobalt resin was visualized by western blot analysis with anti-TopA antibodies (A). The co-eluted His-CobB was stained by Coomassie blue following SDS PAGE (B) or silver on the transfer membrane (C). 


\section{DISCUSSION}

Bacterial DNA topoisomerase I (TopA) is involved in various essential DNAcentered processes. TopA is highly efficient for catalyzing the removal of negative DNA supercoiling generated during rapid transcription. The utilization of different sigma factors for transcription of topA gene and the effect of topA mutation on survival following stress challenge in E. coli, indicate the importance of maintaining cellular TopA activity level under different growth conditions for adaptation and survival (Qi et al., 1996, 1997; Weinstein-Fischer et al., 2000). Transcription of topA gene is increased when the negative supercoiling level of DNA is increased (Tse-Dinh, 1985).

The results presented in Chapter One suggest that in addition to the regulation at the transcription level, cellular TopA activity can be significantly affected by the posttranslational modification of lysine acetylation-deacetylation. Our results showed that TopA activity in the total cell lysate is significantly reduced as a result of the deletion of the $\operatorname{cobB}$ deacetylase gene (Figure 19), even though topA mRNA transcription level detected by qPCR analysis is increased (Figure 21), and TopA protein expression level detected by western blot analysis is also increased (Figure 20).

The intracellular concentration of acP in E. coli reaches at least $3 \mathrm{mM}$, and may be as high as $4.5 \mathrm{mM}$ in wild-type cells. Nonenzymatic acP-dependent acetylation is the predominant mechanism of lysine acetylation in E. coli cells. Our results showed that following in vitro acP-dependent acetylation, the activity of acP-acetylated TopA is reduced, and the reduction level of TopA activity is correlated with TopA acetylation (Figure 22). According to previous studies, CobB deacetylates both enzymatic and nonenzymatic acetylation and suppresses global acetylation to a low level (Kuhn et al., 2014; 
Weinert et al., 2013; Weinert et al., 2017). In our study, the NAD ${ }^{+}$-dependent sirtuin lysine deacetylase CobB was incubated with TopA and acP to mimic the situation that acetylation and deacetylation happen simultaneously in the cell. Our results showed that CobB can partially remove the lysine modification on TopA and maintain the TopA activity at a higher level (Figure 23). The interaction of TopA and CobB was previously revealed by a microarray assay using bio-layer interferometry (Liu et al., 2014). Our result confirmed the CobB and TopA interaction (Figure 24), and it was saturated at 1:50 TopA/CobB molar ratio. The pull-down experiment of TopA and CobB interaction further confirm the deacetylation action of CobB on TopA.

In Chapter One, our hypothesis that acetylation reduces TopA activity is confirmed. Acetylation on TopA reduces its catalytic activity. In vivo and in vitro results demonstrated the significance of TopA acetylation-deacetylation in regulating TopA activity and expression. The results presented here support modulation of TopA catalytic activity via lysine acetylation, and a physiological function of E. coli CobB in preventing TopA inactivation from excess lysine acetylation. 


\title{
CHAPTER TWO: DEACETYLATION OF TOPOISOMERASE I IS AN IMPORTANT PHYSIOLOGICAL FUNCTION OF E. COLI COBB
}

\begin{abstract}
Escherichia coli topoisomerase I (TopA) is reversibly modified by $\mathrm{N}^{\varepsilon}$-Lysine acetylation. We have previously showed that acetylation reduces TopA catalytic activity in vitro and in vivo. However, the physiological significance of TopA acetylation is still unknown. In this chapter, we investigated the global effect of TopA acetylation in DNA supercoiling and cell growth rate, and the specific activity of TopA and the corresponding degree of TopA acetylation during growth transition and growth arrest phases. In addition to the reduction in TopA catalytic activity, we found that the absence of $\mathrm{CobB}$ in a $\triangle \operatorname{cob} B$ mutant increased negative DNA supercoiling. The slow growth phenotype of the $\Delta c o b B$ mutant can be partially compensated by further increase of intracellular TopA level via overexpression of recombinant TopA. The specific activity of TopA expressed from Histagged fusion constructed in the chromosome was inversely proportional to the degree of in vivo lysine acetylation during growth transition and growth arrest. These findings demonstrated that prevention of TopA inactivation from excess lysine acetylation and consequent increase in negative DNA supercoiling is an important physiological function of the CobB protein deacetylase.
\end{abstract}




\section{INTRODUCTION}

E. coli topoisomerase I (TopA), an enzyme encoded by topA gene, regulates global and local DNA supercoiling. The level of supercoiling affects various DNA-centered processes, including DNA replication, transcription, recombination, and transposition (Drlica, 1992; Sobetzko, 2016; Usongo and Drolet, 2014; Wang and Harshey, 1994). DNA supercoiling itself is constantly affected by transcription (Liu and Wang, 1987), as well as changes in nutrient availability and growth environment (Balke et al., 1987; Cheung et al., 2003; Goldstein et al., 1984; Hsieh et al., 1991). The homeostatic state of unconstrained DNA supercoiling is maintained mainly by the relaxation action of TopA and the supercoiling action of DNA gyrase (Drlica, 1992; Zechiedrich et al., 2000). Transcription of topA is increased when DNA is more negatively supercoiled while transcription of gyrA and $g y r B$ is increased by DNA relaxation (Menzel and Gellert, 1983; Tse-Dinh, 1985). In addition, topA gene has four transcriptional initiation sites corresponding to promoters Px1, P1, P2 and P4 under the control of multiple sigma factor including $\sigma^{70}, \sigma^{32}$ and $\sigma^{\mathrm{s}}$ (Qi et al., 1997). Sigma factors are general transcription factors that mediate transcription initiation of all genes via reversibly binding to the $\sigma$ subunit of RNA polymerase (Davis et al., 2017). The activities of the four promoters are growth phase dependent: promoter P2 and P4 are the major topA promoters in exponential phase, and promoter Px1 is the major promoter during stationary phase. Promoter Px1 activity is regulated by the sigma factor $\sigma^{\mathrm{s}}$. Its activity increases upon the entry of stationary phase. While the activity of promoter P4 and $\mathrm{P} 2$ are regulated by $\sigma^{70}$, and decreased in the stationary phase. $\mathrm{P} 1$ is a heat shock promoter recognized by $\sigma^{32}$, it is active during heat shock (Lesley et al., 1990). These four topA 
promoters and their associated sigma factors revealed that the transcription initiation of topA is altered in response to changes in growth conditions (Qi et al., 1997).

The relaxation of transcription-driven negative supercoiling by TopA is important for the response to stress challenge. After the cell is transferred to high temperature, DNA quickly becomes more negatively supercoiled (Goldstein et al., 1984). The transcription of topA is upregulated during the heat shock (Richmond et al., 1999). The DNA is resupercoiled by DNA gyrase, the re-supercoiling effect of gyrase is affected by the heatshock protein DnaK (Ogata et al., 1996) and the histone-like protein HU (Malik et al., 1996; Ogata et al., 1997). The treatment with hydrogen peroxide or N-ethylmaleimide of exponential phase and stationary phase $E$. coli cells both induce topA transcription, which may account for the transient relaxation effect observed after oxidative stress treatment (Tse-Dinh, 2000; Weinstein-Fischer et al., 2000). TopA function has been shown to be important for survival following acid stress. Loss of TopA function sensitizes the cell to acidic stress. topA mutant affects the $\sigma^{\mathrm{s}}$ dependent acid resistance system and the glutamate decarboxylase (GAD) acid resistance system (Stewart et al., 2005).

TopA function is also involved in antibiotics challenges. Treatment with antibiotics such as trimethoprim and mitomycin C induces the bacterial SOS response by causing DNA damage (Liu et al., 2011; Da Re et al., 2009; Singh et al., 2010). SOS response is a bacterial DNA repair system regulated by an inducer RecA and a repressor LexA (Figure 25). Under normal condition, LexA repressor protein binds to SOS promoter element called SOS-box or LexA-box to prevent RNA polymerase binding and gene expression. When DNA damage occurs, the activation of RecA inducer and the autocleavage of LexA 
repressor induce the SOS genes expression. Once the DNA damage is repaired, the RecA filament is no longer pesent and LecA is resynthesized so that the SOS response is inhibited (Andersson and Hughes, 2014; Janion, 2008). TopA function is required for transcriptional activation of recA and $\operatorname{din} D 1$ promoter during SOS response (Liu et al., 2011). RecA can in turn stimulate TopA relaxation activity through direct interaction (Banda et al., 2016). The bactericidal effect of antibiotics is enhanced by the accumulation of TopA-DNA cleavage complex during SOS response (Liu et al., 2011). Moreover, TopA interactions with RNA polymerase during induction of SOS response are likely to influence the degree of antibiotics susceptibility (Yang et al., 2015).

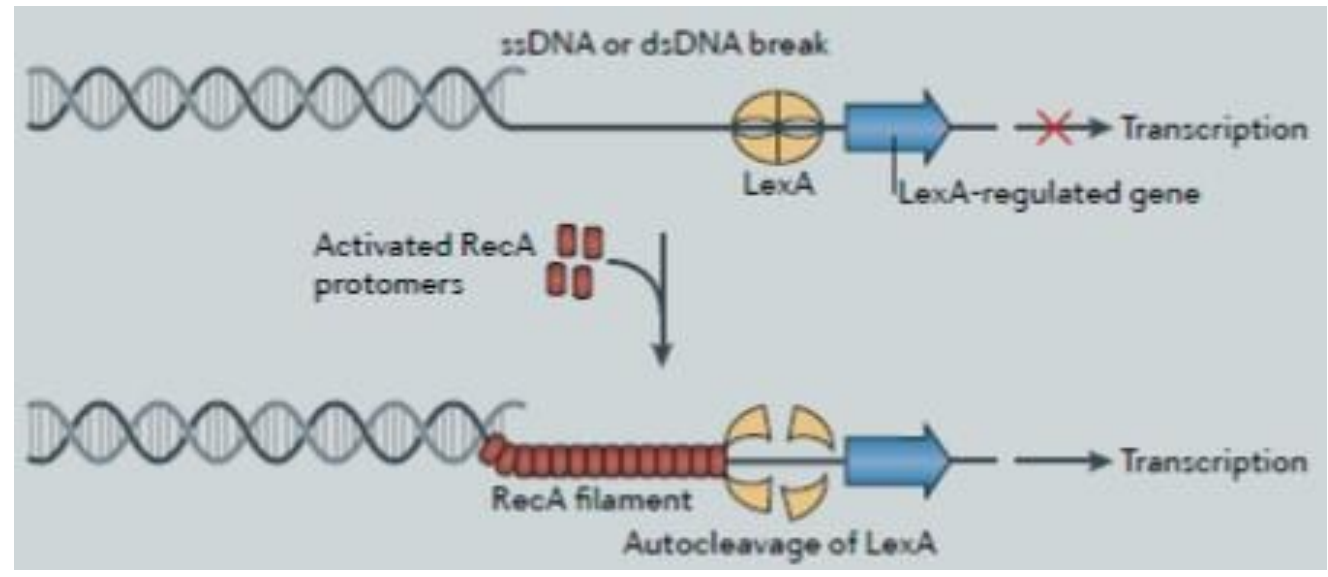

Figure 25 The SOS response (Andersson and Hughes, 2014)

Previous studies demonstrated the significance of bacterial protein acetylation in many cellular processes. Acetylation is important for cells to respond to carbon source overflow (Schilling et al., 2015; Wang et al., 2010; Yu et al., 2008; Zhang et al., 2009a; Zhang et al., 2013a). It adjusts the carbon source utilization pathway by regulating metabolic enzymes (Wolfe, 2016). Acetylation also affect chemotaxis and stress responses. Chemotaxis protein CheY has been revealed to be acetylated by in vivo and in vitro studies (Barak and Eisenbach, 2001; Fraiberg et al., 2015; Li et al., 2010; Liarzi et al., 2010; 
Ramakrishnan et al., 1998; Yan et al., 2008). CheY acetylation shifts the direction of flagellar rotation from counterclockwise to clockwise in response to acetate (Fraiberg et al., 2015).

Proteomics screening identified large number of stress proteins are substrates of lysine acetylation, including heat shock proteins, chaperones, and proteins involved in regulation of free radical reduction (Kim et al., 2006; Zhang et al., 2009a). In the absence of the sirtuin deacetylase CobB, the heat stress resistance and oxidative stress resistance are decreased, while the acid stress resistance is increased (Castano-Cerezo et al., 2014; Ma and Wood, 2011). In addition, deacetylation represses the expression of various stressrelated genes, such as genes related to heat shock, cold shock, osmotic stress and acid resistance, and carbon starvation (Ma and Wood, 2011).

In this chapter, we proposed that TopA acetylation is of physiological significance. To test this hypothesis, we explore the effect of TopA acetylation on DNA supercoiling and cell growth rate. A two dimensional gel analysis was used to assay the supercoiling level of plasmid DNA isolated from wild-type BW25113 and $\Delta c o b B$ mutant JW1106 strains. The growth kinetics of the two strains were also measured in LB medium. TopA expressed and purified from chromosome was used to establish the correlation between degree of lysine acetylation and TopA activity during growth transition and growth arrest. 


\section{MATERIAL AND METHODS}

\section{Materials}

E. coli strains BW25113 [4(araD-araB)567 AlacZ4787(::rrnB-3) $\lambda^{-} r p h-1 \Delta(r h a D-$ rhaB)568 hsdR514], JW1106-1 [4(araD-araB)567 AlacZ4787(::rrnB-3) $\lambda^{-}$ $\triangle c o b B 779:: k a n$ rph-1 $\Delta($ rhaD-rhaB)568 hsdR514] were purchased from the Yale Coli Genetic Stock Center. pETOP plasmid and pCobB plasmid are derivatives of pBAD/Thio (Invitrogen) expressing recombinant proteins. E. coli cells were cultivated in LB medium or TB7 (10g/L tryptone, $100 \mathrm{mM}$ potassium phosphate, $\mathrm{pH} 7.0)$ medium buffered with $0.4 \%$ glucose.

\section{Two-dimensional chloroquine gel analysis of DNA supercoiling}

Plasmids were isolated from BW25113 or JW1106-1 transformants collected at $\mathrm{OD}_{600}=0.8$ using GeneJET Plasmid Mini prep Kit (Thermo Scientific). The superhelical density of the isolated plasmid DNA (1 $\mu \mathrm{g})$ was compared using 2-dimensional electrophoresis analysis in $0.8 \%$ agarose gels with TAE buffer (40mM Tris-acetate, $\mathrm{pH} 8.0$, 2 mM EDTA). About $1 \mu \mathrm{g}$ plasmid DNA mixed with DNA gel loading buffer $(10 \%$ glycerol, $10 \mathrm{mM}$ EDTA, $\mathrm{pH} 8.0,0.1 \%(\mathrm{v} / \mathrm{v})$ bromophenol blue) was loaded and electrophoresed. The first dimension gel and buffer contained $3 \mu \mathrm{g} / \mathrm{ml}$ chloroquine, and electrophoresis was carried out at $3 \mathrm{~V} / \mathrm{cm}$ for $16 \mathrm{~h}$. The gel was soaked and equilibrated in the running buffer containing $25 \mu \mathrm{g} / \mathrm{ml}$ chloroquine for at least $5 \mathrm{~h}$. For the second dimension electrophoresis, the gel was rotated $90^{\circ}$ in the gel tank and electrophoresed at $1.5 \mathrm{~V} / \mathrm{cm}$ for $20 \mathrm{~h}$. To remove chloroquine, the gel was washed by shaking in TAE buffer 
for $2 \mathrm{~h}$ with the buffer replaced every $30 \mathrm{~min}$. The gel was then stained with ethidium bromide and visualized with UV light (AlphaImager Mini, ProteinSimple).

\section{Growth curve analysis}

Plasmid $\mathrm{pBAD} /$ Thio vector, plasmid $\mathrm{pCobB}$ and plasmid $\mathrm{pETOP}$ were transformed into BW25113 or JW1106 chemically competent cells generating three sets of transformants: BW25113 transformed with vector pBAD/Thio and JW1106 transformed with vector $\mathrm{pBAD} /$ Thio; BW25113 transformed with plasmid pCobB and JW1106 transformed with plasmid pCobB; BW25113 transformed with plasmid pETOP and JW1106 transformed with plasmid pETOP. Plasmid pBAD/Thio is a high copy number plasmid and the CobB or TopA coding sequence cloned into the plasmid is under the control of arabinose inducible $\mathrm{P}_{\mathrm{Bad}}$ promoter (Guzman et al., 1995). To perform the growth kinetics, fresh LB medium containing no arabinose or $0.0001 \%$ arabinose was inoculated with overnight culture at 1:100 dilution. Cells were cultured with continuous shaking at $37^{\circ} \mathrm{C}$ for 20 hours to 25 hours. Optical densities at a wavelength of $600 \mathrm{~nm}$ were measured at indicated time points. Growth curves were generated by Graphpad Prime 5.

\section{Construction of strain YN1434 expressing His-tagged TopA from chromosome}

E. coli strain YN1434 expressing His-tagged TopA from chromosome was constructed in the laboratory of our collaborator Dr. Ding Jin in three steps. (i) Two DNA fragments (I and II), which overlap $84 \mathrm{bp}$ at the junction of kanR and the N-terminus of TopA, were amplified by polymerase chain reactions (PCR) using genomic DNA from a strain that contains a selectable KanR marker as DNA template. The primers sequences used for making fragment I and II were listed in Table 3. Primer F1 and R1 covered the sequences upstream of the topA gene, KanR cassette and histidine-tagged N-terminus of 
TopA. Primer F2 and R2 covered the sequences of the junction of kanR, histidine-tagged $\mathrm{N}$-terminus of TopA and additional topA. (ii) Combining the two DNA fragments I and II as DNA template and using the F1 and R2 primers, a PCR fragment was made to cover the sequences of the two fragments. (iii) The chromosomal topA region of the E. coli MG1655 strain (Genotype: F-, $\lambda, r p h-1$ ) was replaced with the long PCR DNA fragment using the standard phage lambda Red-mediated recombination system by selection of kanR marker (Datsenko and Wanner, 2000). The recombinants were validated by PCR assays.

\begin{tabular}{|c|c|}
\hline Primer & Primer sequence (5' to 3') \\
\hline $\begin{array}{l}\text { Forward } \\
\text { primer } 1 \\
\text { (F1) }\end{array}$ & $\begin{array}{l}\text { 5'-GAGCGAGTCCATATCGGTAACTCGTTGCCAGTGGAAGGTT } \\
\text { TATCAACGTGGTGTAGGCGGAGCTGCTTC-3' }\end{array}$ \\
\hline $\begin{array}{l}\text { Reverse } \\
\text { primer } 1 \\
\text { (R1) }\end{array}$ & $\begin{array}{l}\text { 5'-CGATGACAAGAGCTTTACCCATGGATCCGTGATGGTGATGG } \\
\text { TGATGCGATCCTCTCATATGAATATCCTCCTTAGTTCCTATTC-3' }\end{array}$ \\
\hline $\begin{array}{l}\text { Forward } \\
\text { primer } 2 \\
\text { (F2) }\end{array}$ & $\begin{array}{l}\text { 5'- } \\
\text { GAATAGGAACTAAGGAGGATATTCATATGAGAGGATCGCATCA } \\
\text { CCATCACATCACGGATCCATGGGTAAAGCTCTTGTCATCG-3' }\end{array}$ \\
\hline $\begin{array}{l}\text { Reverse } \\
\text { primer } 2 \\
(\mathrm{~F} 2)\end{array}$ & 5'-CGCGGATGTGACCGACGCTGGA-3' \\
\hline
\end{tabular}

Table 3 Sequences of primers used in YN1434 strain construction 


\section{Purification and characterization of chromosomally encoded His-tagged TopA during growth transition and growth arrest}

TB7 broth (10 g/L tryptone, $100 \mathrm{mM}$ potassium phosphate, $\mathrm{pH}$ 7.0) supplemented with $0.4 \%$ glucose was inoculated with overnight culture of strain YN1434 at 1:750 dilution. Cells were cultured with shaking at $37^{\circ} \mathrm{C}$ for $4.5 \mathrm{~h}\left(1.5 \mathrm{~L}\right.$ of culture at $\left.\mathrm{OD}_{600}=0.7\right)$, $5.5 \mathrm{~h}\left(750 \mathrm{~mL}\right.$ of culture at $\left.\mathrm{OD}_{600}=1.7\right), 24 \mathrm{~h}\left(500 \mathrm{~mL}\right.$ of culture at $\left.\mathrm{OD}_{600}=1.9\right)$. His-TopA expressed from the chromosome was purified from the cell pellets using GE Ni Sepharose columns with the addition of the protease inhibitor PMSF $(1 \mathrm{mM})$ and CobB inhibitor nicotinamide $(10 \mathrm{mM})$ to the lysis buffer. Based on Coomassie blue staining of purified His-TopA on the SDS-PAGE gel, equal amounts of His-TopA $(1 \mu \mathrm{g})$ purified from the three growth stages were electrophoresed in $10 \%$ SDS PAGE gel and transferred onto nitrocellulose membrane. The His-TopA on the membrane was stained with the MemCode (Thermo Scientific) reversible protein stain kit before western blot analysis with antibodies against acetylated lysine. The signals were quantified using the Image Studio Digits Ver 4.0 software.

Relaxation activities of purified chromosomally encoded His-TopA were assayed in the $20 \mu \mathrm{l}$ standard reaction volume $(10 \mathrm{mM}$ Tris- $\mathrm{HCl}, \mathrm{pH} 8.0,50 \mathrm{mM} \mathrm{NaCl}, 0.1 \mathrm{mg} / \mathrm{ml}$ gelatin, $6 \mathrm{mM} \mathrm{MgCl}$, $150 \mathrm{ng}$ of supercoiled $\mathrm{pBAD} /$ Thio plasmid DNA). After $30 \mathrm{~min}$ incubation at $37^{\circ} \mathrm{C}$, the reaction was stopped by addition of $4 \mu 1$ stop buffer $(50 \mathrm{mM}$ EDTA, $50 \%$ glycerol, $0.5 \%(\mathrm{v} / \mathrm{v})$ bromophenol blue). The relaxation products were electrophoresed in $1 \%$ agarose gel with TAE buffer (40mM Tris-acetate, 2 mM EDTA) and visualized under UV light. 


\section{RESULTS}

\section{Loss of CobB leads to increase in DNA supercoiling}

The sirtuin deacetylase CobB suppresses protein acetylation E. coli (Weinert et al., 2013; Weinert et al., 2017). Proteomics analysis has revealed an increased acetylation in $\triangle \operatorname{cobB}$ cells (Weinert et al., 2013; Weinert et al., 2017). In previous chapters, we have shown that acetylation reduced the activity of $E$. coli TopA. Considering the important function of TopA in maintaining the level of DNA supercoiling, we proposed that acetylation of TopA has an impact DNA supercoiling. To investigate the effect of lysine acetylation on DNA supercoiling, the DNA supercoiling level of plasmids extracted from wild-type BW25113 and $\triangle \operatorname{cobB}$ mutant JW1106 was measured by two dimensional chloroquine gel. Chloroquine is a DNA intercalator that relaxes negative supercoiled DNA and introduces positive supercoils. Wild-type BW25113 and $\triangle \operatorname{cobB}$ mutant JW1106 transformed with plasmid $\mathrm{pBAD} /$ Thio were grown at $37^{\circ} \mathrm{C}$ until $\mathrm{OD}_{600}$ reached 0.8 . The extracted DNA plasmids were electrophoresed in agarose gel in the presence of $3 \mu \mathrm{g} / \mathrm{ml}$ chloroquine in the first dimension and $25 \mu \mathrm{g} / \mathrm{ml}$ chloroquine in the second dimension to resolve the positively and negatively supercoiled DNA topoisomers. The expected distributions of positive supercoiled DNA and negatively supercoiled DNA were shown in Figure 26C. Plasmid DNA isolated from $\triangle c o b B$ mutant was more negatively supercoiled than that from wild-type cell (Figure 26A). When pCobB plasmid was introduced to the wild-type and $\triangle \operatorname{cobB}$ mutant, the supercoiling level of pCobB plasmid from the wild-type and $\triangle \operatorname{cobB}$ mutant was identical (Figure 26B). These results indicated that the loss of CobB increases DNA negative supercoiling. The increased DNA negative supercoiling is consistent with the reduced TopA relaxation activity shown in Chapter One (Figure 18). 
Since TopA is the major relaxation function to maintain the DNA supercoiling level, reduction in TopA relaxation activity because of the absence of CobB would result in the increases in DNA negative supercoiling.

A

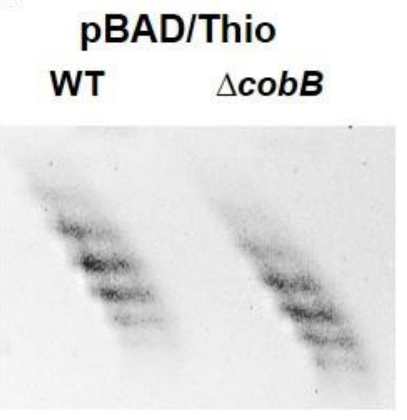

C

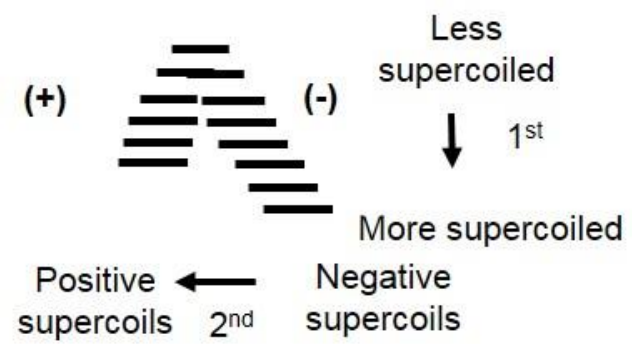

B pCobB $\Delta$ cobB WT

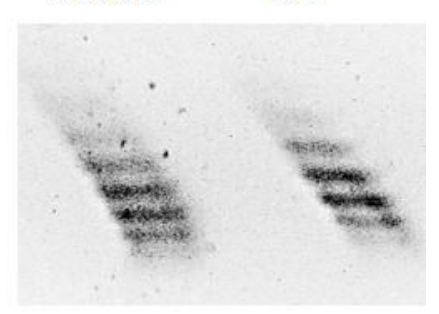

Less

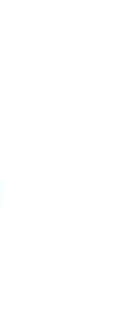

Figure 26 2-Dimensional chloroquine gel analysis comparing DNA supercoiling in BW25113 (wildtype) and JW1106 ( $\triangle \operatorname{cobB}$ ) mutant strains (Zhou et al., 2017)

Plasmids $\mathrm{pBAD} /$ Thio and $\mathrm{pCobB}$ were extracted from cultures at $\mathrm{OD}_{600}=0.8$. The first dimension electrophoresis was carried out in $0.8 \%$ agarose gel with TAE buffer containing $3 \mu \mathrm{g} / \mathrm{ml}$ chloroquine at $3 \mathrm{~V} / \mathrm{cm}$ for $16 \mathrm{~h}$. The agarose gel was then rotated 90 degrees for electrophoresis in the second dimension with $25 \mu \mathrm{g} / \mathrm{ml}$ chloroquine in the TAE buffer. The schematic diagram shows the expected distribution of positively and negatively supercoiled DNA topoisomers following electrophoresis 


\section{Overexpression of recombinant TopA partially compensates the delayed growth of the $\triangle \operatorname{cobB}$ mutant}

The growth kinetics of wild-type BW25113 and $\triangle \operatorname{cobB}$ mutant JW1106 transformed with $\mathrm{pBAD} /$ Thio plasmid was monitored continuously for $24 \mathrm{~h}$ in LB culture grown with vigorous shaking at $37^{\circ} \mathrm{C}$. The $\triangle \operatorname{cob} B$ mutant strain grew much slower during the exponential phase when compared to the wild-type strain (Figure 27A, B). The reduction of cellular TopA catalytic activity as well as increased negative supercoiling observed in the $\triangle \operatorname{cobB}$ mutant might account in part for its slow growth phenotype. Plasmid $\mathrm{pETOP}$ or $\mathrm{pCobB}$ derived from the $\mathrm{pBAD} /$ Thio plasmid were introduced into both strains for growth rate analysis under the same conditions. Plasmid pETOP and pCobB are high copy number plasmid that can overexpress recombinant TopA and CobB at the basal level or by arabinose induction (Guzman et al., 1995). While wild-type cells with either $\mathrm{pBAD} /$ Thio or $\mathrm{pETOP}$ displayed similar growth rates, the delayed growth of the $\Delta c o b B$ mutant was partially rescued by the presence of pETOP (Figure 27). The result indicates that reduction of TopA activity when acetylation-deacetylation regulation is perturbed is of physiological significance. Overexpression of recombinant CobB from the pobB plasmid also partially rescued the slow growth of the $\Delta$ cobB mutant (Figure 27A), with greater degree of complementation in the presence of $0.0001 \%$ arabinose to further induce the expression of recombinant CobB (Figure 27B). 

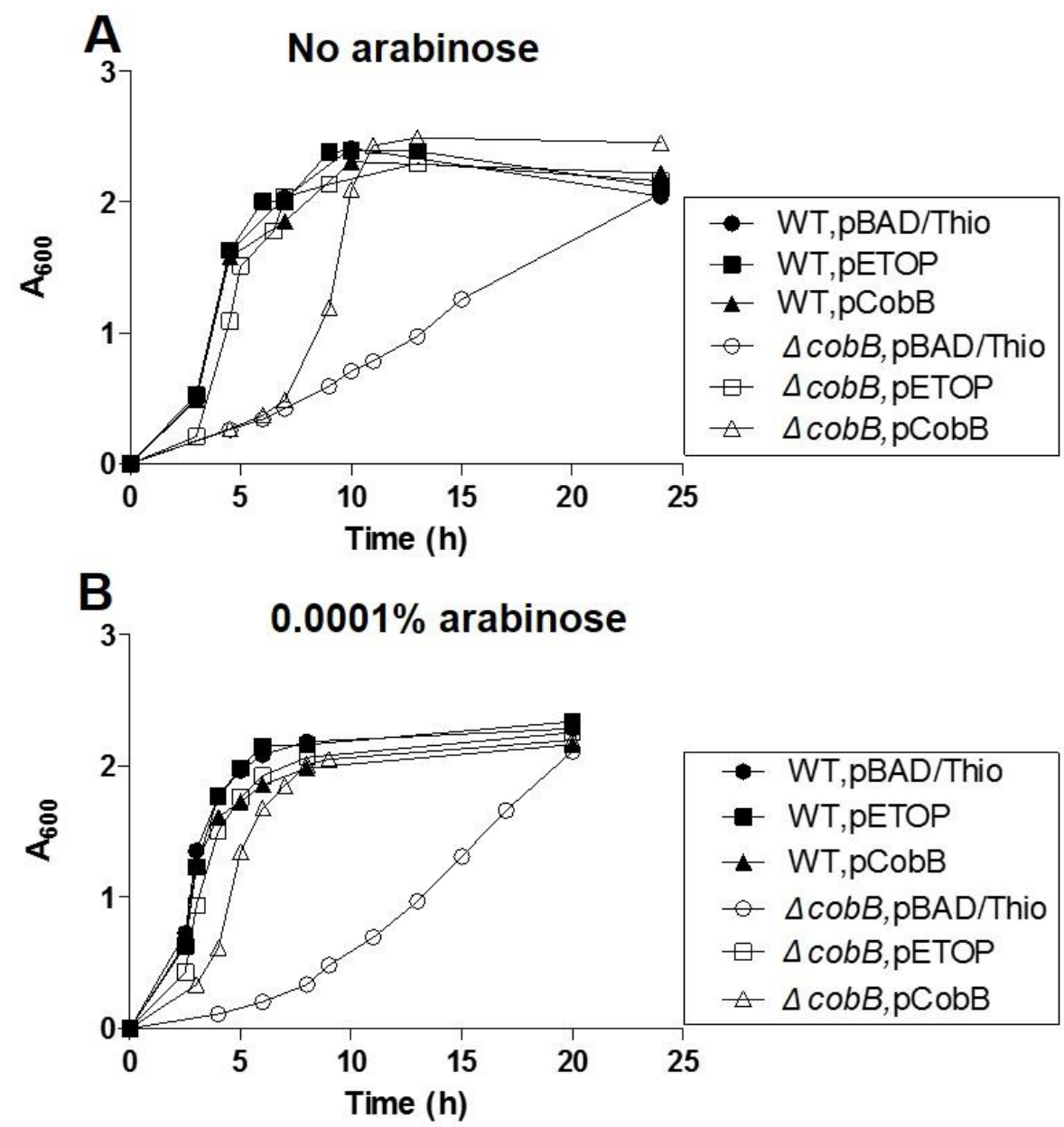

Figure 27 Effect of recombinant TopA overexpression on the slow-growth phenotype of the $\triangle \operatorname{cob} B$ mutant (Zhou et al., 2017)

(Filled circle) Wild-type BW25113 strain transformed with pBAD/Thio; (Unfilled circle) $\triangle \operatorname{cobB}$ JW1106 strain transformed with pBAD/Thio; (Filled square) Wild-type strain transformed with pETOP for TopA overexpression; (Unfilled square) $\triangle \operatorname{cob} B$ mutant strain transformed with pETOP; (Filled triangle) Wildtype strain transformed with pCobB; (Unfilled triangle) $\triangle \operatorname{cob} B$ mutant strain transformed with pCobB. The LB medium contained no arabinose (A) or $0.0001 \%$ arabinose (B). 


\section{Specific activity of His-TopA expressed from the chromosome is inversely}

proportional to degree of lysine acetylation during growth transition and growth arrest

To demonstrate directly the effect of in vivo lysine acetylation on the specific activity of TopA, we utilized E. coli strain YN1434 that expresses TopA with N-terminal His-tag from the chromosome. Cells were collected from cultures in buffered TB7 broth supplemented with $0.4 \%$ glucose following growth for $4.5 \mathrm{~h}\left(\mathrm{OD}_{600}=0.7\right), 5.5 \mathrm{~h}\left(\mathrm{OD}_{600}=1.7\right)$ and growth arrest at $24 \mathrm{~h}\left(\mathrm{OD}_{600}=1.9\right)$. Acetyl phosphate-dependent acetylation has been shown to occur mostly following entry into stationary phase under these experimental conditions (Kuhn et al., 2014; Schilling et al., 2015). The His-TopA was purified with Niaffinity chromatography and analyzed for level of lysine acetylation (Figure 28A) and specific relaxation activity using supercoiled plasmid DNA as substrate (Figure 28B). The results showed that the level of lysine acetylation found in the purified His-TopA first decreased as cells entered stationary phase at $5.5 \mathrm{~h}$. and then increased during growth arrest at $24 \mathrm{~h}$ (Figure 28A). The specific activity of the purified His-TopA was found to be inversely proportional to the degree of lysine acetylation (Figure 28B). The His-TopA purified from the growth arrest (GA) culture had the lowest activity ( $\sim 2$ fold lower than $\mathrm{OD}_{600}=0.7$ culture, and $4-8$ fold lower than $\mathrm{OD}_{600}=1.7$ culture). This further confirmed that TopA catalytic activity can be modulated in vivo based on the degree of lysine acetylation modification of the enzyme. 


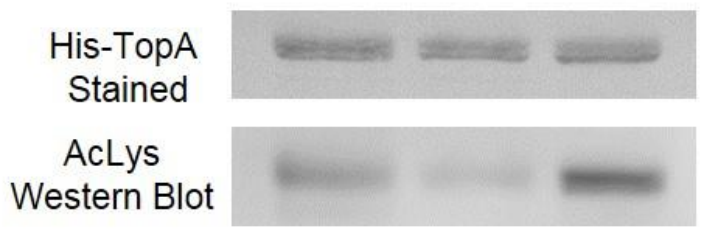

\begin{tabular}{|l|c|c|c|c|}
\hline & acK Signal & His-TopA Signal & acK/His-TopA & Relative Ratio \\
\hline OD 0.7 $(4.5 \mathrm{~h})$ & 1160 & 191 & 6.1 & 2.9 \\
\hline OD 1.7 $(5.5 \mathrm{~h})$ & 324 & 152 & 2.1 & 1.0 \\
\hline GA $(24 \mathrm{~h})$ & 2220 & 157 & 14.1 & 6.6 \\
\hline
\end{tabular}

B

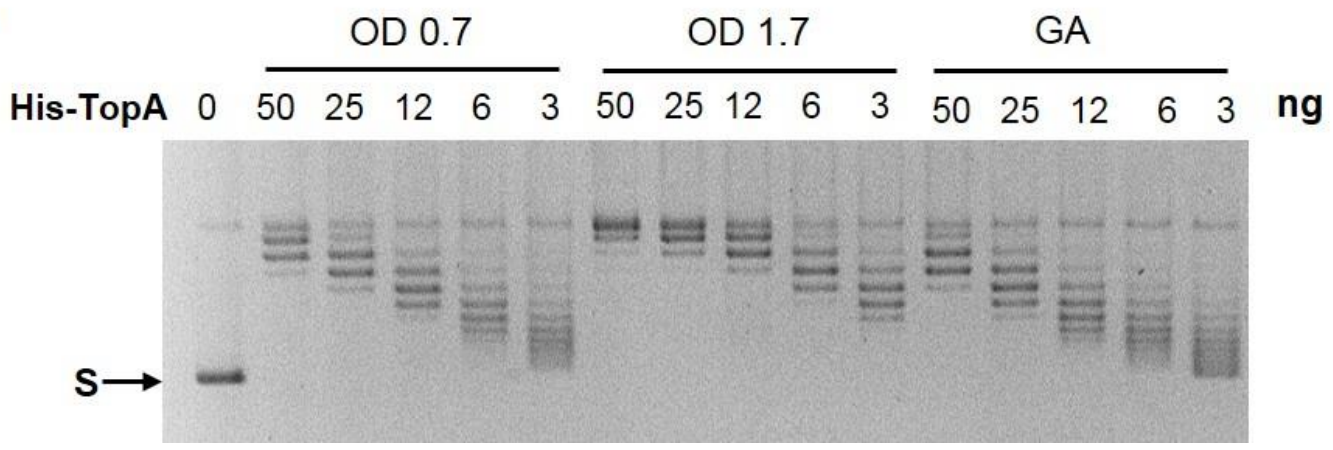

Figure 28 Topoisomerase I specific activity is inversely proportional to degree of lysine acetylation during growth transition and growth arrest. (Zhou et al., 2017)

(A) Western blot analysis of purified chromosomal His-TopA using monoclonal anti acetyllysine antibody. His-TopA expressed from chromosome in YN1434 strain at exponential phase (OD600 0.7), entry into stationary phase (OD600 1.7) and growth arrest (GA) was purified by Ni Sephorase column. Acetylation level of $1 \mu \mathrm{g}$ purified His-TopA from OD600 0.7, OD600 1.7 and GA was detected by western blot analysis using antibody against acetyl lysine (AcK). His-TopA on the membrane was visualized by MemCode reversible staining. The signals of His-TopA staining was used to normalize the acK signal for comparison of degree of lysine acetylation. (B) Serial dilution of His-TopA (50ng, 25ng, $12 \mathrm{ng}$, 6ng, 3ng) was incubated with $150 \mathrm{ng}$ supercoiled plasmid DNA (S) at $37^{\circ} \mathrm{C}$ for $30 \mathrm{~min}$ to assay for catalytic activity. 


\section{DISCUSSION}

In Chapter One, we have demonstrated the effect of acetylation on TopA catalytic activity and TopA expression level. To further understand the physiological significance of TopA acetylation, the global DNA supercoiling, growth kinetics and TopA acetylation level were analyzed here. We extracted plasmid from wild-type BW25113 and $\triangle c o b B$ mutant JW1106, and analyzed the DNA supercoiling of the plasmids using two dimensional chloroquine gel. The results showed that in addition to reducing TopA activity and increasing TopA expression, acetylation on TopA has a global impact on DNA topology. DNA supercoiling level of plasmid isolated from $\triangle \operatorname{cobB}$ mutant is more negatively supercoiled than that isolated from the wild-type cell (Figure 26A). Our results also revealed the importance of CobB deacetylation in maintaining DNA supercoiling level. When the $\triangle \operatorname{cobB}$ mutation is complemented by pCobB, DNA supercoiling levels are identical when both wild-type and $\triangle \operatorname{cobB}$ mutant were introduced with plasmid pCobB (Figure 26B). Moreover, the higher level of topA expression in the $\Delta \operatorname{cobB}$ mutant observed in Figure 20 is likely due to the stimulation of topA transcription (Figure 21) by the increase in negative supercoiling in the homeostatic regulation of DNA supercoiling. Here, we propose a simple model to explain how acetylation regulates TopA expression. The reduction of TopA relaxation activity by acetylation increases the global DNA supercoiling,

which in turn upregulates topA gene transcription and TopA protein expression. In a previous study of global response to loss of DNA supercoiling (Peter et al., 2004), cobB transcription was found to be repressed by inhibition of DNA gyrase or gyrB mutation. Based on the effect of the $\Delta c o b B$ mutation on DNA supercoiling observed here, decreased CobB activity in the presence of gyrase mutation or inhibitor may contribute to countering 
the effect of reduced gyrase supercoiling activity via reduction of TopA relaxation activity for the homeostatic regulation of DNA supercoiling.

Lysine acetylation affects multiple cellular functions, including cell motility, carbon source utilization central metabolism, and stress responses (Barak and Eisenbach, 2001; Castano-Cerezo et al., 2014; Ma and Wood, 2011; Schilling et al., 2015; Wang et al., 2010). Previous proteomics studies identified a large number of acetylated proteins in E. coli (Schilling et al., 2015; Weinert et al., 2013; Yu et al., 2008; Zhang et al., 2009a; Zhang et al., 2013a). In this report, we observe a slow growth phenotype in entering the exponential phase associated with the $\Delta \operatorname{cobB}$ mutation. Nutrient should not be limiting in the LB medium under the growth condition of the experiment. The slow growth phenotype could potentially be due to the global effect of acetylation-deacetylation on a wide range of $E$. coli proteins. Interestingly, when the $\triangle \operatorname{cobB} B$ mutant is complemented with a high copy number plasmid expressing recombinant TopA, the slow growth phenotype is partially reversed. This could be due to the global effect of TopA via its influence on DNA supercoiling. TopA activity has been reported to be important for the removal of localized transcription-driven negative supercoiling at the ribosomal operon $\operatorname{rrnB}$ (Masse et al., 1997). Reduction in TopA catalytic activity as a result of the $c o b B$ deletion may lead to hypernegative supercoiling at the highly transcribed rRNA loci as the cells exit the growth arrest to enter the exponential phase under the culture conditions used in this study.

We further established the correlation between degree of lysine acetylation and specific activity of topoisomerase I by purifying His-tagged TopA expressed from the chromosome following growth transition and growth arrest. Non-enzymatic acetylation of TopA by acetyl phosphate is expected to be highest at growth arrest following $24 \mathrm{~h}$ 
incubation in buffered TB7 media supplemented with glucose. Acetylation of TopA in the growth arrest phase was accumulated to the highest level, acetylation level of TopA in exponential phase $\left(\mathrm{OD}_{600}=0.7\right)$ was lower than that in growth arrest phase (24h growth, $\left.\mathrm{OD}_{600}=1.9\right)$ but higher than the level of TopA acetylation in growth transition phase $\left(\mathrm{OD}_{600}=1.7\right)$. Our results support previous studies that protein acetylation accumulates in growth arrest phase (Weinert et al., 2013; Yu et al., 2008; Zhang et al., 2009a). Lysine acetylation may play an important role in controlling protein functions in response to environmental signals. Upon transferring culture in growth arrest phase into fresh medium, most proteins were deacetylated within 2 hours (Yu et al., 2008). Here, we have observed a similar pattern of the change in acetylation level. The TopA acetylation level decreased during exponential phase and early stationary phase, then increased in growth arrest phase. The corresponding relaxation activities of purified His-TopA were inversely proportional to the degree of TopA acetylation. Further studies are needed to determine the basis for the decrease in TopA acetylation that was observed during the initial transition from exponential phase to stationary phase. 


\title{
CHAPTER THREE: INVESTIGATION OF MOLECULAR MECHANISM OF $E$. COLI TOPOISOMERASE I ACTIVITY REDUCTION BY ACETYLATION
}

\begin{abstract}
TopA relaxation activity is shown here to be reduced by lysine acetylation in vitro and in vivo. Acetylation of TopA affects DNA supercoiling and cell growth rate. However, the mechanism of TopA activity reduction by acetylation is still unknown. In this chapter, the TopA relaxation mechanism, specifically the strand passage step, was investigated first. Nuclease footprinting assay was used to illustrate the interaction of TopA C-terminal domains with T-strand DNA. Crosslinking of TopA to the phenylselenyl-modified thymidine incorporated in T-strand help to identify amino acid residues that may be involved in strand passage. Here, we showed that TopA interacts with both G-strand and T-strand DNA in a double chain model. The N-terminal domains of TopA interact with the G-strand while the C-terminal domains of TopA interacts with the T-strand. Five lysine residues are crosslinked to the modified thymidine suggesting these sites may correspond to proximal sites for the unwound DNA strand during catalysis. Mass spectrometry analysis of acetyl phosphate acetylated TopA identified abundant acetylated lysine residues providing candidate lysine residues for site-directed mutagenesis. To model the effect of lysine acetylation, selected lysine residues are mutated to alanine to mimic the effect of positive charge removal. Our results showed that substitution of Lys-484 with alanine reduces the relaxation activity, which may contribute to the TopA activity reduction from acetylation observed in previous chapters.
\end{abstract}




\section{INTRODUCTION}

E. coli topoisomerase I (TopA) is essential for removal of hypernegative supercoiling of DNA. The crystal structure of $67 \mathrm{kDa} \mathrm{N}$-terminal fragment of TopA (TOP67) elucidates the amino acids residues in domains D1-D4 that are involved in the cutting and rejoining of DNA during enzyme catalysis. TopA cleaves, shuffles and rejoins DNA through the active site tyrosine residue at position 319 (Tyr 319) of the enzyme (Feinberg et al., 1999; Lima et al., 1993). In the crystal structure of TOP67, Tyr 319 interacts with several residues to carry out the efficient DNA relaxation. Glu-9 present in the TOPRIM motif (Aravind et al., 1998) interacts with 3' oxygen of the scissile phosphate, which is critical for DNA breakage and rejoining (Chen and Wang, 1998). The cluster of three acid residues Asp-111, Asp-113 and Glu-115 also located in TOPRIM motif is important for the binding of $\mathrm{Mg}$ (II) required for DNA relaxation (Zhu and Tse-Dinh, 2000). His-365, located near the active site Tyr 319, participate in DNA binding and is important for catalysis at physiological pH (Perry and Mondragon, 2002). Arg-321 interacts with the scissile phosphate of DNA, is required for positioning of the phosphotryrosine linkage in DNA rejoining (Chen and Wang, 1998; Narula et al., 2011). Three amino acid residues Arg-169, Arg-173 and Tyr-177 interact with DNA upstream from the DNA cleavage site

and provide sequence selectivity of a cytosine residue at the -4 position relative to the cleavage site (Narula and Tse-Dinh, 2012).

However, the N-terminal fragment of TopA alone cannot catalyze the relaxation of negatively supercoiled DNA. The C-terminal domains (D5-D9) also participates in negative supercoiling removal (Ahumada et al., 1998). Three repetitive Zinc (II) binding 
domains (D5-D7) are necessary for strand passage and has been proposed to interact with T-strand DNA during catalysis because the deletion of these domains deactivates TopA relaxation (Ahumada and Tse-Dinh, 1998, 2002). Overexpression of the recombinant 14 $\mathrm{kDa}$ C-terminal fragment (D8-D9) of TopA was toxic to the cell, probably due to the interaction of recombinant C-terminal fragment of TopA with the $\beta$ ' subunit of RNA polymerase abolishes the physiological interaction between RNA polymerase and full length TopA via its C-terminus domains D5-D9 (Cheng et al., 2003; Yang et al., 2015; Zhu et al., 1995). The structure-based modeling of the TopA and RNA polymerase interactions predicts amino acid residues Arg-609, Lys-627 and Lys-664 are involved in the formation and the stabilization of the TopA-RNAP complex (Tiwari et al., 2016).

In previous proteomics studies, 29 lysine residues out of 70 total lysines in TopA have been shown to form mass spectrometry detectable acetylated lysines (Figure 29). These lysine residues are located across the entire length of TopA. Several predominant lysine residues are identified to be acetylated in most conditions by different studies, including Lys 144, Lys 346, Lys 484 (Weinert et al., 2013; Yu et al., 2008; Zhang et al., 2009a; Zhang et al., 2013a). A lysine residue Lys-788 was found to have $77 \%$ stoichiometry of acetylation (Baeza et al., 2014). Acetylation on lysine residues may have an impact on TopA partial activities such as DNA binding, cleavage, religation, as well as the overall catalytic activity and TopA interactions. 


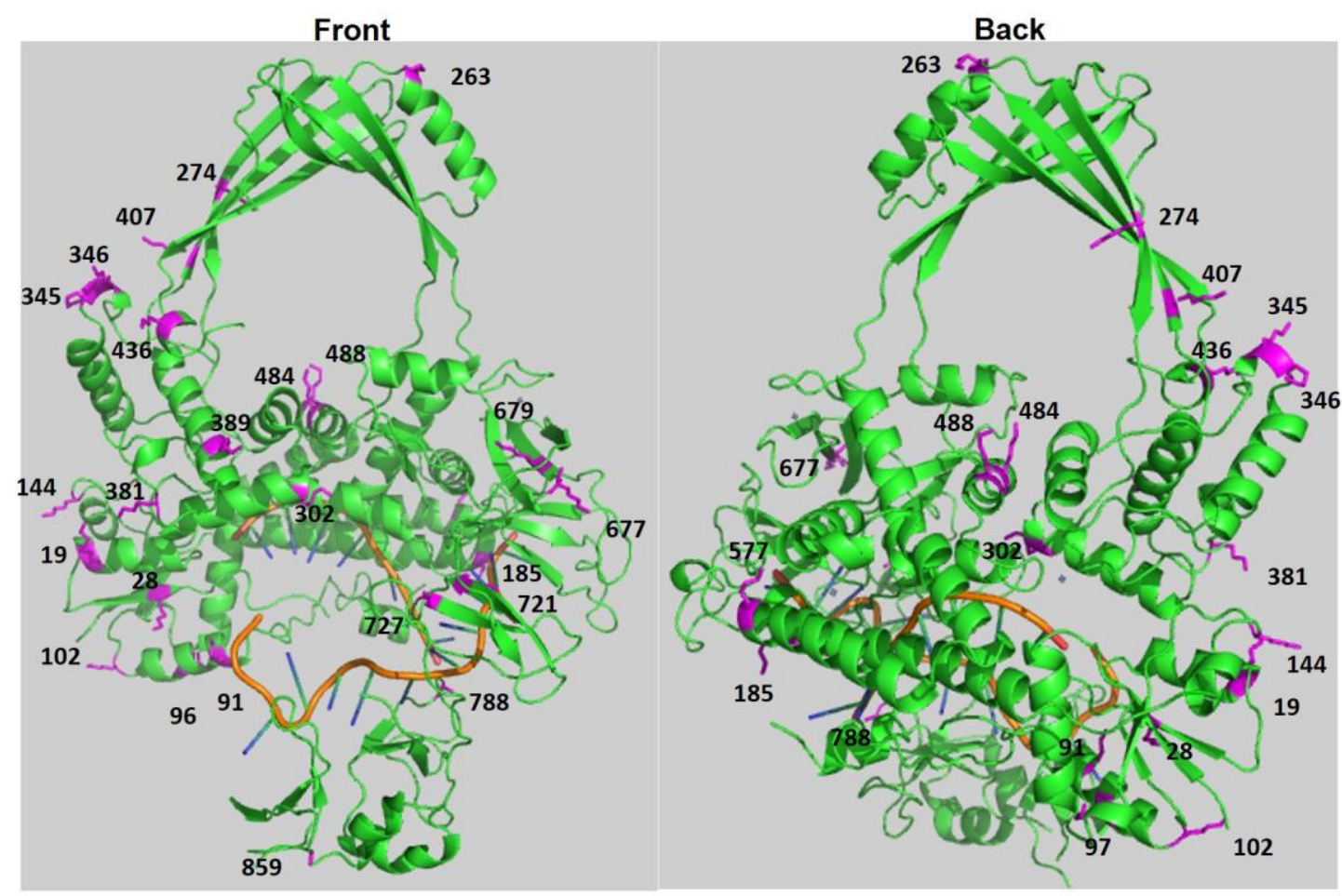

Figure 29 Positions of lysine acetylation in full length TopA as complex with two bound singlestranded DNA segments based on its crystal structures (Tan et al., 2015, Lima et al., 1993)

Results described in this chapter addresses the molecular mechanism of TopA activity reduction following lysine acetylation. In order to study the effect of individual lysine acetylation, the knowledge gap of how TopA guides the T-strand DNA during catalysis was studied first. A full length crystal structure of TopA (PDB 4RUL) was obtained in collaboration with Dr. Kemin Tan (Argonne National Lab) (Tan et al., 2015). Nuclease footprinting assay was used to map the interaction TopA C-terminal domains with the T-strand DNA. DNA crosslinking experiment was used to identify interactions between specific amino acid residues of TopA and DNA which are not shown by the available crystal structure. We found that nonenzymatic acetyl phosphate mediated acetylation reduces TopA catalytic activity in the previous chapter. Here, mass 
spectrometry analysis of the in vitro acetyl phosphate acetylated TopA identified the acetylated lysine residues which may contribute to the activity reduction following acetylation. Selected lysine residues were substituted with alanine by site-directed mutagenesis to mimic the state of lysine acetylation. The relaxation activities of purified mutant TopA proteins was assayed to study the effect of individual lysine acetylation.

\section{MATERIALS AND METHODS}

\section{Materials}

The oligonucleotide Bubble Substrate is a partial duplex synthesized by SigmaAldrich (Figure 30A). The oligonucleotide Substrate-Y (Figure 30B) containing two single- stranded DNA segment linked to a double-stranded stem was provided by Dr. Greenbergas (Johns Hopkins University). G-strand of the Substrate-Y was hybridized with T-strand which contains a reactive group PhSe incororporated at the 5' end of the T-strand (PhSeT). Radionucleotides $\left[\gamma^{32} \mathrm{P}\right]$ ATP was used to radiolabel the oligonucleotide substrate

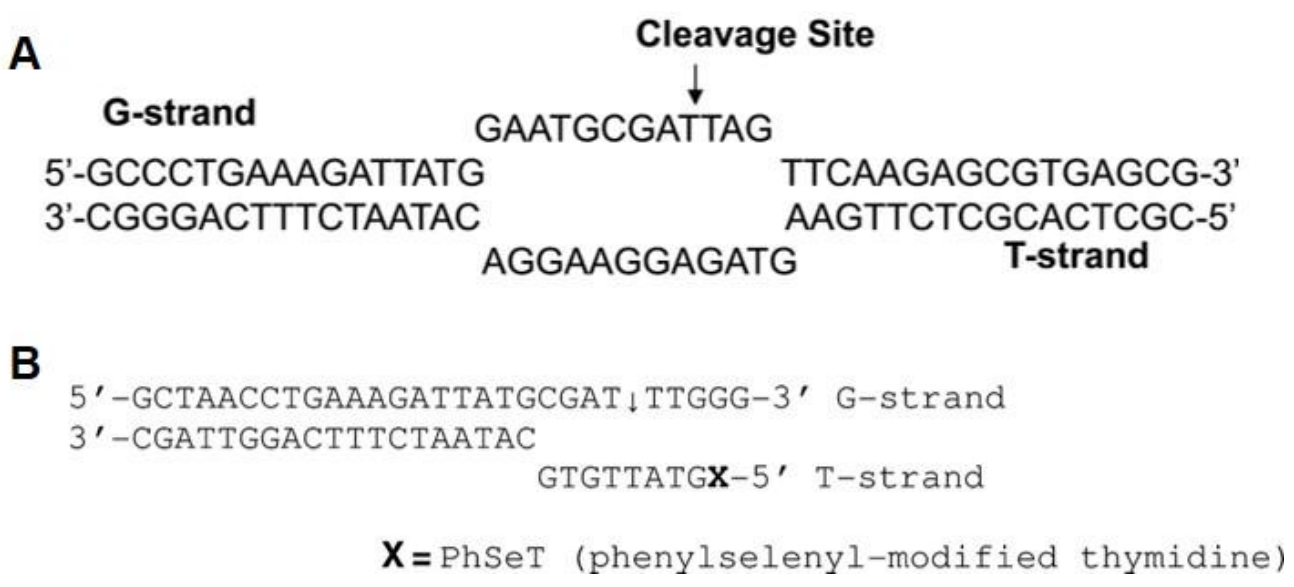

Figure 30 Oligonucleotide substrates

(A) Sequence of DNA Bubble structure (Tan et al., 2015). (B) Sequence of DNA Substrate-Y (Cheng et al., 2017). 
with T4 polynucleotide kinase (New England Biolabs). Micro Bio-Spin chromatography columns (Bio-Rad) and Sephadex G-50 (GE) were used to clean up the radiolabeled oligonucleotides. Wild-type TopA and mutant TopA proteins are expressed from a recombinant plasmid pLIC-EcTOP (Doyle, 2005). Primers used for site-directed mutagenesis are listed in Table 4.

\section{Nuclease footprinting of TopA binding to the bubble substrate}

E. coli TopA G116S mutant was purified and reported in previous study (Cheng et al., 2005). TopA G116S mutant forms a stabilized irreversible covalent complex after DNA cleavage allowing the monitor of TopA binding. A bubble-substrate mimicking underwound DNA was designed to have a single TopA cleavage site in the bubble region of the G-strand (Figure $30 \mathrm{~A}$ ). The bottom strand has no cytosine base in the bubble region, so it acts as the T-strand (Narula and Tse-Dinh, 2012). The G-strand or T-strand was labeled at the 5' end with $\left[\gamma^{32} \mathrm{P}\right]$ ATP by T4 polynucleotide kinase (New England Biolabs). The radiolabeled strand was hybridized with the other strand at $90^{\circ} \mathrm{C}$ for 10 min followed by cooling down overnight to form the bubble substrate. Increasing concentrations of TopA G116S were incubated with the bubble substrate in $40 \mathrm{mM}$ Tris- $\mathrm{HCl}, 0.1 \mathrm{mg} / \mathrm{ml} \mathrm{BSA}, 5$ $\mathrm{mM} \mathrm{MgCl} 2$ for $1 \mathrm{~h}$ at $37^{\circ} \mathrm{C} .5 \mathrm{ng}$ of DNase I (New England Biolabs) or $7.5 \mathrm{pg}$ of Micrococcal nuclease (New England Biolabs) was incubated with the protein-substrate complex for $2 \mathrm{~min}$ or $1 \mathrm{~min}$ at $37^{\circ} \mathrm{C}$ to digest unbound substrate region. Nuclease digestion was stopped by addition of $50 \mathrm{mM}$ EDTA. The reaction products were electrophoresed in a 15\% sequencing gel, and analyzed by Pharos FX Plus Phosphorimager (Bio-Rad). 


\section{Mass spectrometry analysis to Identify TopA lysines crosslinked to Substrate-Y}

TopA was crosslinked to unlabeled Substrate $\mathrm{Y}$ in crosslinking buffer $(20 \mathrm{mM}$ $\mathrm{NaH}_{2} \mathrm{PO}_{4}, 100 \mathrm{mM} \mathrm{NaCl}, 1 \mathrm{mM}$ EDTA, $\mathrm{pH}$ 7.2). The crosslinking reaction was initiated by adding $5 \mathrm{mM} \mathrm{NaIO}_{4}$ and followed by incubating at room temperature in the dark for 2 hours. The crosslinking reaction was stopped by the incubation with $50 \mathrm{mM} \mathrm{Na} 2 \mathrm{SO}_{3}$ at room temperature for $5 \mathrm{~min}$. After crosslinking of Substrate-Y with TopA, the crosslinking product was enriched by DE52 cartridge with diethylaminoethyl resin (GE) and eluted with buffer containing $250 \mathrm{mM} \mathrm{NaCl}$. After dialysis, the crosslinked Substrate-Y was digested by $10,000 \mathrm{U}$ Micrococcal nuclease at $37^{\circ} \mathrm{C}$ for 1 hour. The digestion products were electrophoresed by SDS 5-10\% gradient polyacrylamide gel, and visualized by Coomassie blue staining. The gel band containing crosslinked TopA and unreacted TopA was excised and submitted to the Proteomics and Mass Spectrometry Facility and the University of Massachusetts Medical School for in-gel digestion with trypsin and LC/MS/MS analysis.

\section{Mass spectrometry analysis of acetyl-phosphate acetylated TopA}

$0.1 \mu \mathrm{g} / \mu \mathrm{l}$ purified $E$. coli TopA was incubated with $5 \mathrm{mM}$ acP at $37^{\circ} \mathrm{C}$ in $150 \mathrm{mM}$ Tris- $\mathrm{HCl}(\mathrm{pH} 8.0), 10 \%$ glycerol, and $10 \mathrm{mM} \mathrm{MgCl}_{2}$ for 4 hours. The acetylation reaction was stopped by adding an equal volume of 2X SDS loading buffer and then boiling for 10 min. $15 \mu \mathrm{g}$ of acetylated TopA and $15 \mu \mathrm{g}$ of control unacetylated TopA were electrophoresed by $10 \%$ SDS PAGE gel and visualized by Coomassie blue staining. Gel bands were excised and subjected to in-gel digestion with trypsin (Thermo Scientific). The

mass spectrometry part was carried out by Advance Mass Spectrometry Facility in FIU. Mass spectrometry analysis was conducted in a Bruker Impact HD ESI-QTOF instrument 
in the reflectron mode and in the positive $(+)$ ion polarity using nano-ESI direct infusion with an $\mathrm{ACN} / \mathrm{H}_{2} \mathrm{O}$ solvent with $0.1 \%$ Formic Acid solvent (Beck et al., 2015). Mass spectrometry data was analyzed utilizing the Bruker Daltonics Data Analysis software suite equipped with Bruker Biotools for the analysis of the post-translational modifications. Peptide mass fingerprinting identification was performed using the open access MASCOT search utilizing the SwissProt Database. Searches were performed allowing for 2 missed cleavages, and a peptide mass tolerance of $+/-0.1 \mathrm{ppm}$ with carbamidomethyl (C) and acetyl $(\mathrm{K})$ fixed modifications and oxidation $(\mathrm{M})$ variable modifications.

\section{Site-directed mutagenesis}

Mutations were introduced at indicated locations with primers listed in Table 4. Plasmid pLIC-ETOP was used as the template for PCR amplification (Doyle, 2005). QuickChange site-directed mutagenesis protocol and Q5 site-directed mutagenesis protocol were used. With QuickChange Site-directed protocol, topA mutant clones were amplified using Q5 High-fidelity DNA polymerase (New England Biolabs) in following cycling conditions: $95^{\circ} \mathrm{C}$ for $30 \mathrm{~s}$; followed by 35 cycles of $95^{\circ} \mathrm{C}$ for $20 \mathrm{~s}$, annealing temperature varied by primers for $20 \mathrm{~s}, 72^{\circ} \mathrm{C}$ for $4 \mathrm{~min} ; 72^{\circ} \mathrm{C}$. After PCR amplification, the template strand was digested by Dpn 1 (New England Biolabs) at $37^{\circ} \mathrm{C}$ for $1 \mathrm{~h}$. The Dpn1 digested plasmids were transformed into NEB $5 \alpha$ competent cells (New England Biolabs). With Q5 Site-directed mutagenesis protocol, topA mutant clones were amplified using Q5 hot start high-fidelity master mix in following conditions: $98^{\circ} \mathrm{C}$ for $30 \mathrm{~s} ; 25$ cycles of $98^{\circ} \mathrm{C}$ for $10 \mathrm{~s}$, varied annealing temperature for $20 \mathrm{~s}, 72^{\circ} \mathrm{C}$ for $4 \mathrm{~min} ; 72^{\circ} \mathrm{C}$ for $5 \mathrm{~min}$. After

PCR amplification, Kinase, Ligase and DpnI (KLD) treatment was performed to circularize 
the PCR products and digest the DNA template. The mutant plasmids were transformed into NEB $5 \alpha$ competent cells. Transformants were selected by plating on LB plates containing $50 \mu \mathrm{g} / \mathrm{ml}$ kanamycin. Plasmids were extracted from colonies. The mutation and the complete topA gene were verified by DNA sequencing service from Eurofins and Genewiz.

\begin{tabular}{|l|l|}
\hline Primer name & Primer sequence \\
\hline $\begin{array}{l}\text { TopA K19A } \\
\text { Forward }\end{array}$ & AACGATCAACGCGTATCTGGGTAGTGACTACGTG \\
\hline $\begin{array}{l}\text { TopA K19A } \\
\text { Reverse }\end{array}$ & TTGGCTTTTGCCGGGGAC \\
\hline $\begin{array}{l}\text { TopA K144A } \\
\text { Forward }\end{array}$ & GAGTGGTGTTTAACGAAATTACTGCAAACGCGATCCGCCAG \\
\hline $\begin{array}{l}\text { TopA K144A } \\
\text { Reverse }\end{array}$ & CTGGCGGATCGCGTTTGCAGTAATTTCGTTAAACACCACTC \\
\hline $\begin{array}{l}\text { TopA K153A } \\
\text { Forward }\end{array}$ & GGCATTTAACGCACCGGGTGAGC \\
\hline $\begin{array}{l}\text { TopA K153A } \\
\text { Reverse }\end{array}$ & TGGCGGATCGCGTTTTTA \\
\hline $\begin{array}{l}\text { TopA K346A } \\
\text { Forward }\end{array}$ & $\begin{array}{l}\text { TATATCAGCGATAATTTTGGTAAGGCATATCTGCCGGAAAGTC } \\
\text { CGAATC }\end{array}$ \\
\hline $\begin{array}{l}\text { TopA K346A } \\
\text { Reverse }\end{array}$ & $\begin{array}{l}\text { GATTCGGACTTTCCGGCAGATATGCCTTACCAAAATTATCGC } \\
\text { GATATA }\end{array}$ \\
\hline $\begin{array}{l}\text { TopA K788A } \\
\text { Forward }\end{array}$ & CCAACACTTTCCCGGCATCGCGTGAAACG \\
\hline $\begin{array}{l}\text { TopA K788A } \\
\text { Reverse }\end{array}$ & CGTTTCACGCGATGCCGGGAAAGTGTTGG \\
\hline $\begin{array}{l}\text { TopA K484A } \\
\text { Forward }\end{array}$ & TTCAGTGAAGCATCGCTGGTTGCAGAGCTGGAAAAACGCG \\
\hline $\begin{array}{l}\text { TopA K484A } \\
\text { Reverse }\end{array}$ & CGCGTTTTTCCAGCTCTGCAACCAGCGATGCTTCACTGAA \\
\hline
\end{tabular}

Table 4 Primers used in site-directed mutagenesis 


\section{Expression and purification of wild TopA and mutant TopA}

Wild-type E. coli TopA and mutant TopA clones generated by site-directed mutagenesis were transformed into $E$. coli BL21 DE3 competent cells (Invitrogen). The pLIC-ETOP plasmid has the EcTOP1 coding sequence under the control of the T7 promoter with a protease cleavable N-terminal His6-tag. The T7 promoter is under the control of isopropyl $\beta$ - D-1- thiogalactopyranoside (IPTG) inducible lac promoter (Doyle, 2005). Protein expression in E. coli strain BL21 DE3 is also under the control of lacI promoter. Recombinant wild-type TopA and mutant TopA were induced at $37^{\circ} \mathrm{C}$ for 4 hours with the addition of $1 \mathrm{mM}$ IPTG to culture when $\mathrm{OD}_{600}$ reached 0.4 . Cell pellets were centrifuged after 4 hours induction and lysed by lysozyme treatment in lysis buffer $(50 \mathrm{mM}$ $\mathrm{NaH}_{2} \mathrm{PO}_{4}, 0.3 \mathrm{M} \mathrm{NaCl}, 10 \mathrm{mM}$ imidazole, $\mathrm{pH}$ 8.0) on ice for 1 hour and three freeze-thaw cycles. The soluble lysates were obtained as the supernatant fractions following ultracentrifugation at $31,000 \mathrm{rpm}$ at $4^{\circ} \mathrm{C}$ for 2 hours. The supernatant containing the recombinant proteins was loaded onto the self-packed Ni Sepharose 6 Fast Flow (GE) column, then washed with wash buffer $\left(50 \mathrm{mM} \mathrm{NaH}_{2} \mathrm{PO}_{4}, 0.3 \mathrm{M} \mathrm{NaCl}, 20 \mathrm{mM}\right.$ imidazole, $\mathrm{pH}$ 8.0). The bound recombinant protein was eluted with elution buffer $\left(50 \mathrm{mM} \mathrm{NaH}_{2} \mathrm{PO}_{4}\right.$, $0.3 \mathrm{M} \mathrm{NaCl}, 400 \mathrm{mM}$ imidazole, $\mathrm{pH}$ 8.0). Proteins in elution fraction were electrophoresed by $10 \%$ SDS-PAGE gel and visualized by Coomassie blue staining. The elution fraction containing recombinant TopA were combined and dialyzed against storage buffer $(50 \mathrm{mM}$ $\mathrm{NaH}_{2} \mathrm{PO}_{4}, 0.3 \mathrm{M} \mathrm{NaCl}, \mathrm{pH} 8.0,50 \%$ glycerol, $1 \mathrm{mM}$ DTT). When the removal of $\mathrm{N}-$ terminal His ${ }_{6}$-tag is required, elution fractions were dialyzed against Buffer A with $0.1 \mathrm{M}$ $\mathrm{KCl}\left(20 \mathrm{mM} \mathrm{KH}_{2} \mathrm{PO}_{4}, 1 \mathrm{mM}\right.$ EDTA, $0.1 \mathrm{M} \mathrm{KCl}, \mathrm{pH}$ 8.0, $10 \%$ glycerol, $1 \mathrm{mM}$ DTT). The $\mathrm{N}$-terminal His ${ }_{6}$-tag was cleaved by TEV protease treatment at $20^{\circ} \mathrm{C}$ for 4 hours followed 
by overnight incubation at $4^{\circ} \mathrm{C}$. The cleaved His6-tag and TEV protease were removed by passing through Ni Sepharose column. Recombinant proteins recovered in the flowthrough and wash fractions were purified by self-packed single-stranded DNA cellulose (Sigma-Aldrich) column. After washing, increasing concentration of $\mathrm{KCl}$ was used for gradient elution. Elution fractions containing recombinant TopA were dialyzed against storage buffer ( $0.1 \mathrm{M} \mathrm{KH}_{2} \mathrm{PO}_{4}, 0.2 \mathrm{mM}$ EDTA, pH 8.0, $0.2 \mathrm{mM}$ DTT, 50\% glycerol). Protein concentrations were determined using the Bradford Protein Assay (Bio-Rad).

\section{Assay for TopA relaxation activity}

TopA were serially diluted as indicated and assayed for relaxation activity in a standard reaction volume of $20 \mu \mathrm{l}(10 \mathrm{mM}$ Tris- $\mathrm{HCl}, \mathrm{pH} 8.0,50 \mathrm{mM} \mathrm{NaCl}, 0.1 \mathrm{mg} / \mathrm{ml}$ gelatin, $6 \mathrm{mM} \mathrm{MgCl} 2,150 \mathrm{ng}$ of supercoiled $\mathrm{pBAD} /$ Thio plasmid DNA). After $30 \mathrm{~min}$ incubation at $37^{\circ} \mathrm{C}$, the reaction was stopped by addition of $4 \mu 1$ stop solution $(50 \mathrm{mM}$ EDTA, 50\% glycerol, 0.5\% (v/v) bromophenol blue). The DNA was electrophoresed in 1\% agarose gel with TAE buffer (40mM Tris-acetate, $2 \mathrm{mM}$ EDTA) and visualized with UV light. 


\section{RESULTS}

\section{TopA interacts with double-stranded DNA substrate through a double chain model}

As mentioned, in the proposed relaxation mechanism of type IA topoisomerase, TopA binds to and cleaves the G-strand DNA allowing the T-strand to pass through. However, details of how TopA interacts with both G- and T-strand DNA is still not clear. Based on the crystal structure of full length TopA with single-stranded DNA (ssDNA) bound to the C-terminal domains (4RUL) and the previous crystal structure of $67 \mathrm{kDa}$ TopA with ssDNA bound to the N-terminal domains (3PX7) (Zhang et al., 2011), a comprehensive model of TopA bound with two ssDNA segments representing the G-strand and T-strand DNA was generated (Figure 31). To gain more information about TopA on

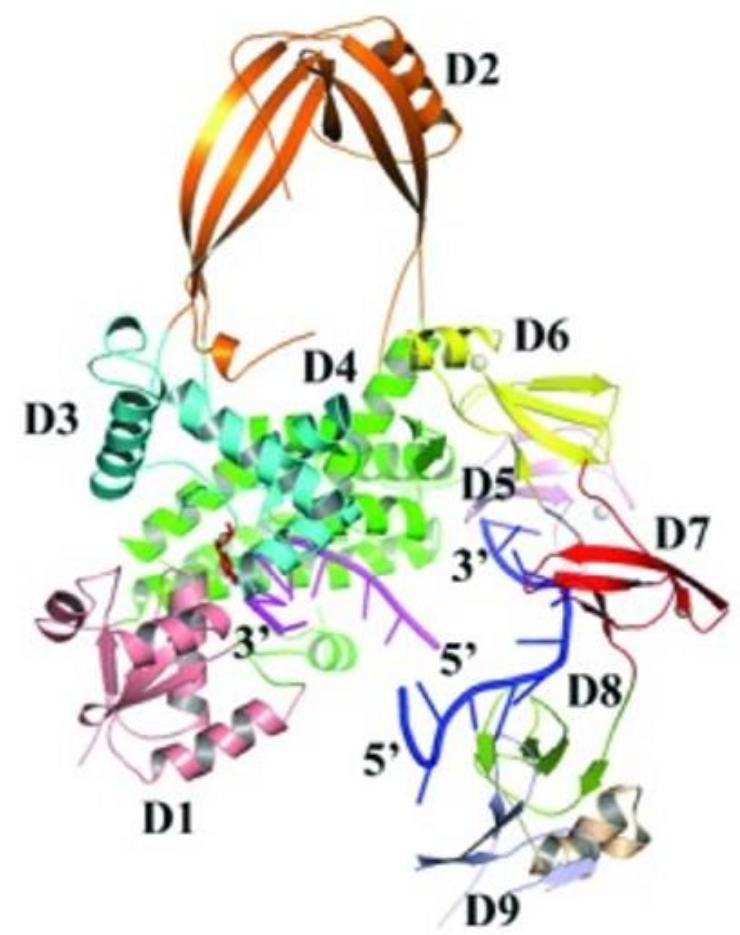

Figure 31. Modeled structure of full length TopA in complex with two ssDNA segments based on two crystal structures each with one bound ssDNA segment (Tan et al., 2015) 
the mechanistic aspect as well as the structural aspect based on the full length TopA structure, two models of TopA binding with G- and T-strand DNA during relaxation were proposed: a single chain model and a double chain model (Figure 32A, B). In the single chain model, TopA only interacts with the G-strand DNA, while in the double chain model, $\mathrm{N}$-terminal domains of TopA interact with the G-strand (Figure 32A) and C-terminal domains interact with the T-strand (Figure 32B).

Nuclease footprint experiments were carried out to test these two models. The Bubble-substrate (Figure 30A) with a 12-nucleotide unpaired region was used. The Gstrand unpaired region was shown in a previous study to be sufficient for TopA binding, cleaving and rejoining (Narula and Tse-Dinh, 2012). The T-strand unpaired region will not be cleaved by TopA because it does not contain cytosine required for TopA cleavage recognition. TopA G116S mutant was used for the footprinting experiment to form a covalent protein-DNA cleavage complex. Protection of the G-strand and T-strand in the Bubble-substrate from DNase I or Micrococcal nuclease (MNase) digestion was monitored by $\left[\gamma^{32} \mathrm{P}\right]$ ATP labeling of the individual strand at the $5^{\prime}$ end. DNase I primarily cleaves double-stranded region of the bubble substrate, while MNase preferentially cleaved the single-stranded region. TopA G116S protein covalent binding with the G-strand resulted in a partial protection of the dsDNA region from DNase I digestion in both the G-strand and T-strand radiolabeled substrates (Figure 33A, B) and a complete protection of the ssDNA region from MNase digestion in both substrates (Figure 34A, B). These results further support the double-chain model shown in Figure 32 that during TopA relaxation, 
N-terminal domains mainly interact with the G-strand DNA segment and C-terminal domains interact with the T-strand DNA.

A

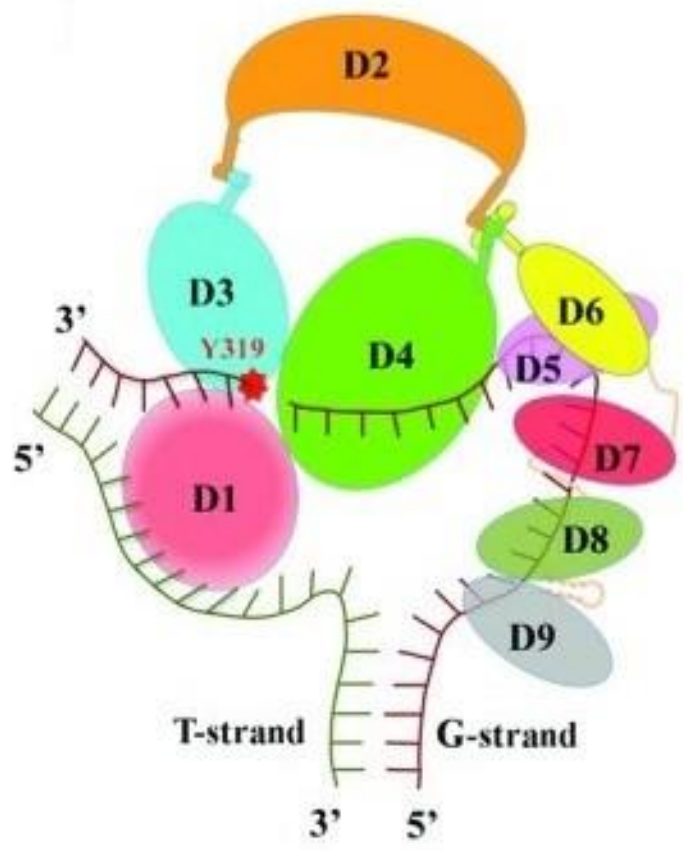

B

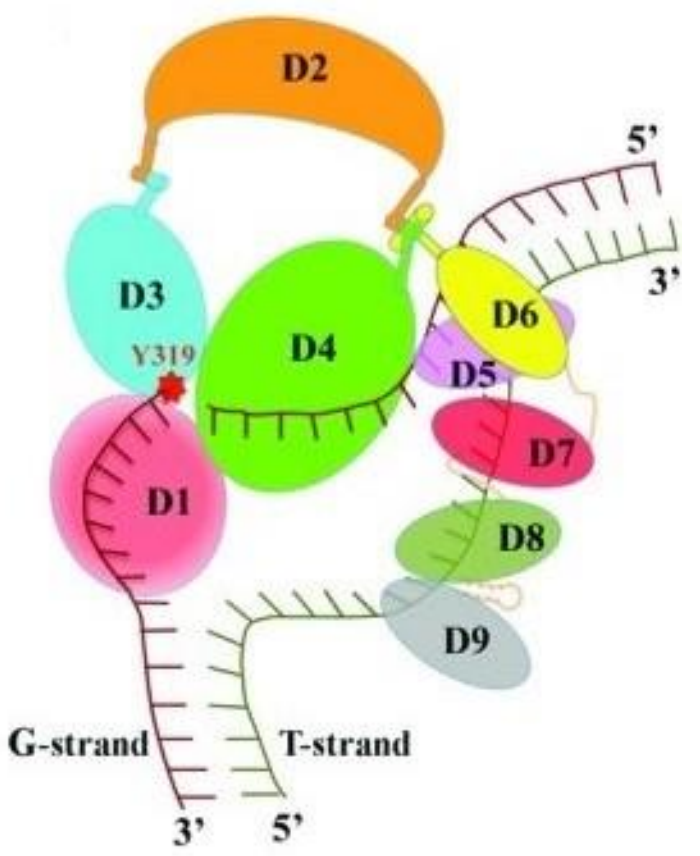

Figure 32 DNA binding models (Tan et al., 2015)

(A) Single chain model. In single chain model, N-terminal and C-terminal domains of TopA primarily bind to the G-stand. (B) Double chain model. In double chain model, N-terminal domains of TopA binds to G-strand, while C-terminal domains of TopA binds to T-strand. D1 does not bind DNA in the crystal structures. 


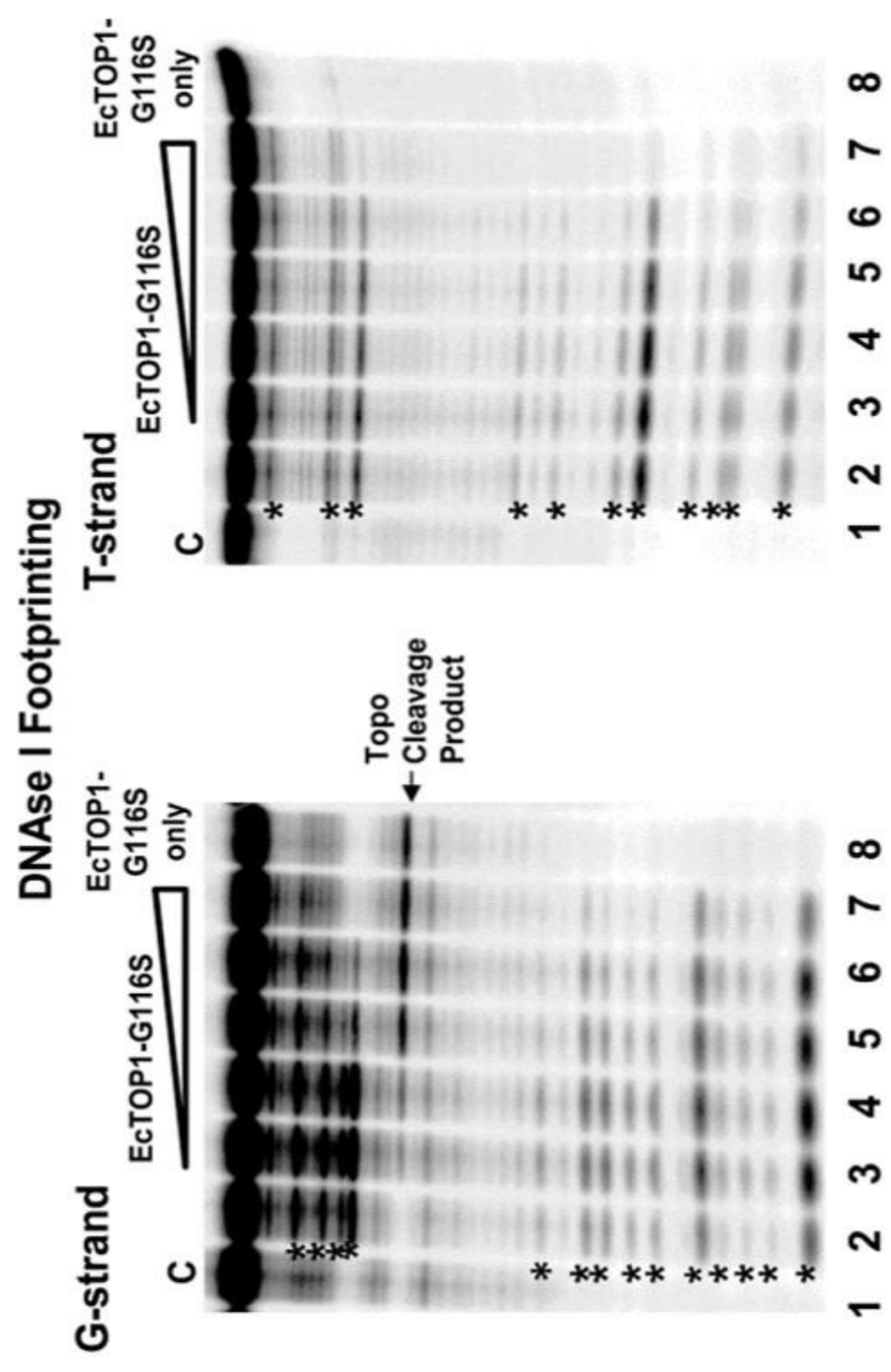

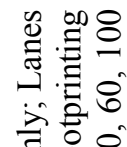

б。 웅

जे

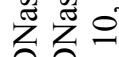

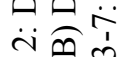

范

范

苛吕齐

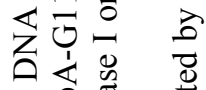

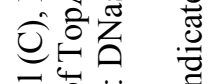

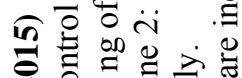

ชิ

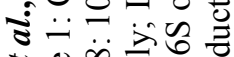

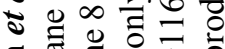

ส

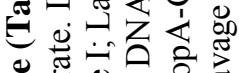

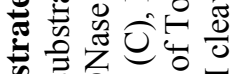

के के

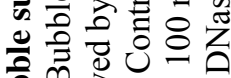

$\infty \quad$ 更

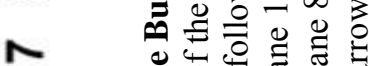

范它击 击

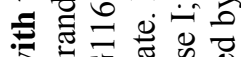

○

م)

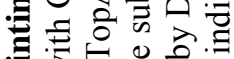

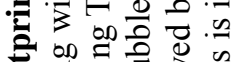

눙.

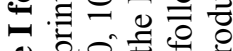

\& 0 过出 出

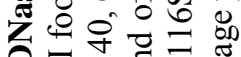

ले

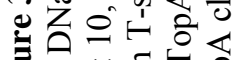

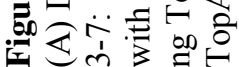




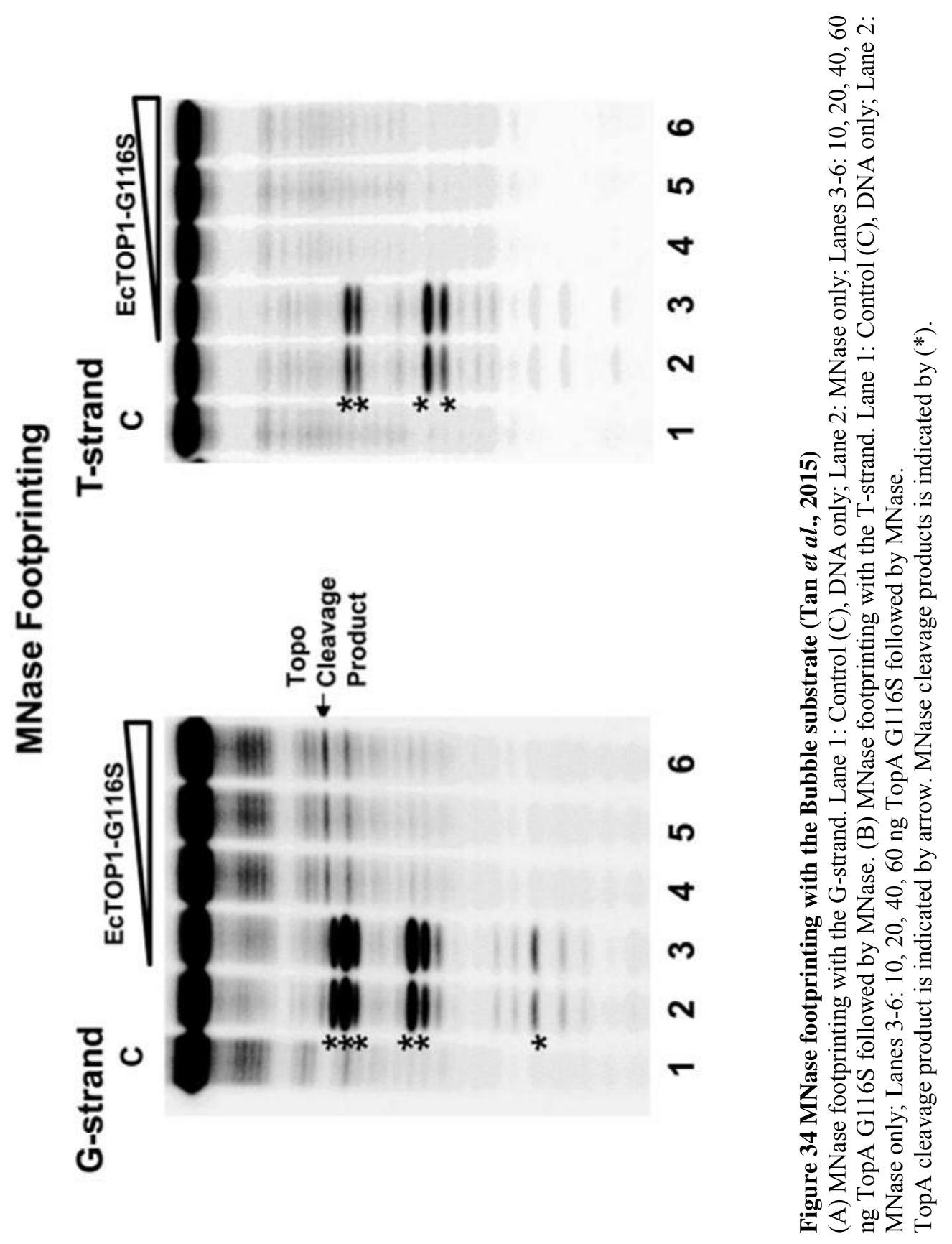




\section{Identification of lysines in TopA crosslinked to T-strand of Y-substrate}

Tryptic digestion and LC/MS/MS analysis were used to identify crosslinked peptides in nuclease treated Substrate Y-TopA complex with the increase of molecular mass 649 as a result of crosslinking to the modified thymidine on the T-strand. The spectral results indicated that lysine residues at positions 19, 28, 279, 303, and 679 of TopA were crosslinked. Positions of these lysine sites are shown in Figure 35A. K19 and K28 are in domain D1, K279 is in domain D2, K303 is in domain D3, and K679 is in domain D6. Histidine681 may also be a potential crosslinking site. Moreover, no cysteines in the crosslinked peptides was identified. K279, located in D2 domain of N-terminus, is proximal to a nucleotide binding site in the central cavity of the enzyme based on the structure of $67 \mathrm{kDa}$ N-terminal TopA in complex with trinucleotides pTTT (PDB 1CY1) (Figure 35B). K679, located in D6 domain of C-terminus, is proximal to the ssDNA based on the crystal structure of full length TopA (PDB 4RUL) (Figure 35C). K28 which is in D1 domain of $\mathrm{N}$-terminus and $\mathrm{K} 303$ which is in $\mathrm{D} 3$ domain of $\mathrm{N}$-terminus are positioned close to the active site tyrosine in the structure of the covalent complex of TopA with a cleaved G-strand DNA (PDB 3PX7) (Figure 35D). 

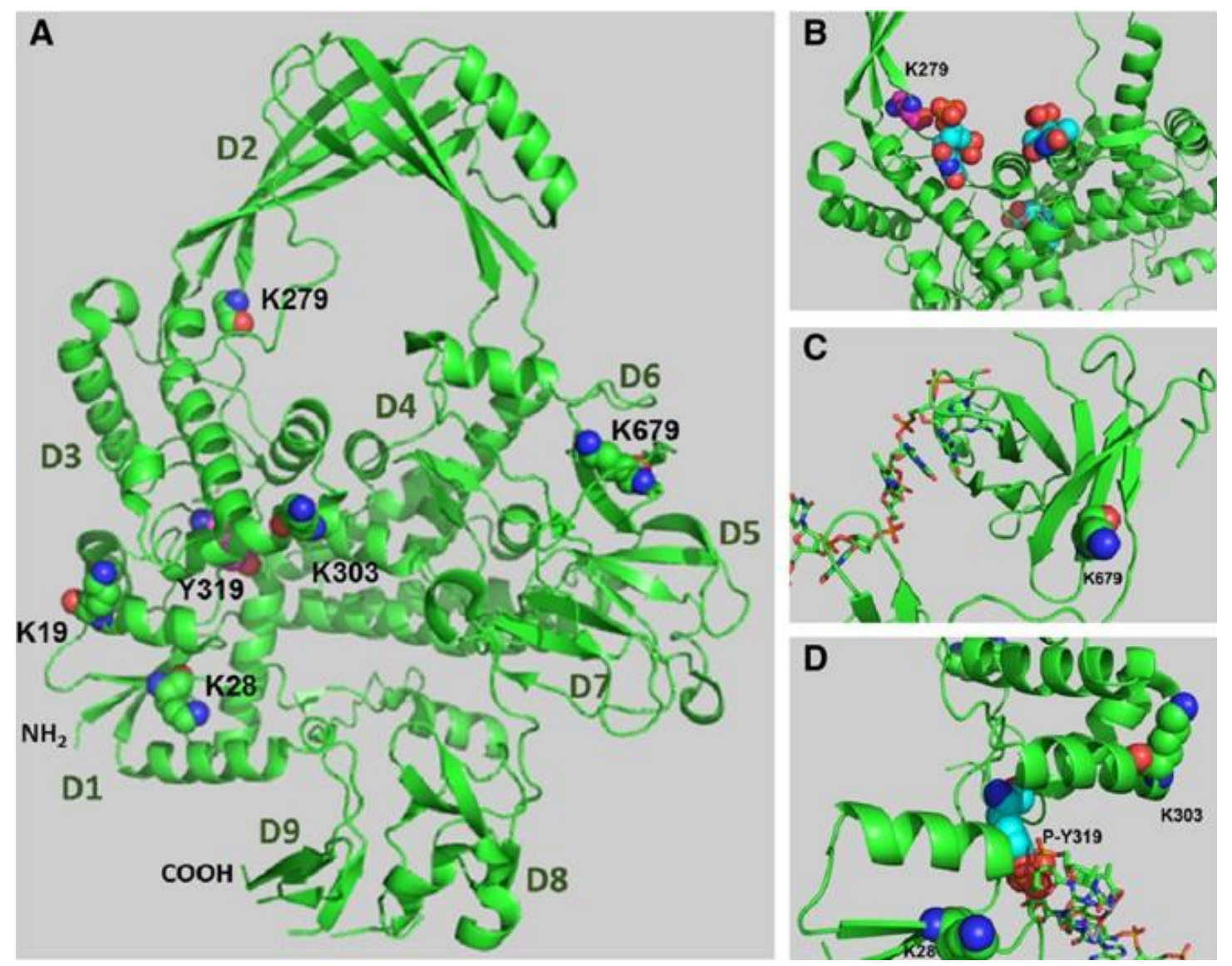

Figure 35 Positions of crosslinked lysine residues in TopA (Cheng et al., 2017)

(A) Locations of crosslinked lysine residues in different domains of full length TopA (PDB 4RUL). (B) A close-up view of K279 in proximal to nucleotide binding sites (PDB 1CY1). (C) A close-up view of K679 in proximal to ssDNA bound to the C-terminus (PDB 4RUL). (D) A close-up view of K28 and K303 positioned close to active site tyrosine and the covalently bound ssDNA (PDB 3PX7). 


\section{Identification of acetylated lysine residues in acP-acetylated TopA}

As we showed in Chapter One, acetyl phosphate (acP) mediated in vitro acetylation of TopA reduced the enzyme catalytic activity. To investigate the acetylated lysine residues of TopA that may cause the activity reduction, mass spectrometry was used to analyze acPacetylated TopA. $15 \mu \mathrm{g}$ of unacetylated TopA and $15 \mu \mathrm{g}$ acetylated TopA were processed in parallel for trypsin digestion and MS analysis. Abundant acetylated lysine sites have been detected (Table 5). Analysis of TopA acetylated by low concentration acetylphosphate identified 15 acetylation sites with $68.2 \%$ sequence coverage. K57, K58 and K61 are located in domain D1; K443 is located in domain D2; K302 and K346 are located in domain D3; K185 is in domain D4; K677, K727, K737 and K741 are located in zinc ribbon domains (domain D5-D7). K810, K836, K859 and K864 are located in the Cterminal domain of TopA (Figure 36A). Analysis of TopA acetylated by high concentration of acetyl-phosphate has identified 44 acetylation sites with $54.6 \%$ sequence coverage. K3, K15, K19, K46, K54, K57, K58, K60, K61, K91, K97, K102, K144 are in domain D1; K302, K407, K423, K443 are located in domain D2; K436 in D3; K212, K484 and K521

are located in domain D4; K605, K659, K664, K727 and K741 are in zinc ribbon domain (domain D5-D7), K752, K810, K834, K844 and K864 are located in C-terminal domain of TopA responsible for DNA binding (Figure 36B). More acetylated lysine sites were found in TopA acetylated by high concentration acP than that by low concentration acP, which was consistent with the reduction of TopA activity shown in Chapter One (Figure 18). 


\begin{tabular}{|l|l|l|}
\hline $\begin{array}{l}\text { acP } \\
\text { concentration }\end{array}$ & $\mathrm{K}^{\mathrm{ac}}$ sites & Sequence coverage \\
\hline Low [acP] & $\begin{array}{l}57,58,61,185,302,346,443,677,727, \\
737,741,810,836,859,864\end{array}$ & $\begin{array}{l}122 \text { peptide hits, } \\
68.2 \% \text { coverage }\end{array}$ \\
\hline High [acP] & $3,15,19,46,54,57,58,60,61,91,97$, & 149 peptide hits, \\
& $\begin{array}{l}102,144,212,302,407,423,436,443, \\
584,521,605,659,664,727,741,752,\end{array}$ & \\
& $810,834,844,864$ & \\
\hline
\end{tabular}

Table 5 Mass spectrometry identified acetylated lysine sites in acP acetylated TopA.
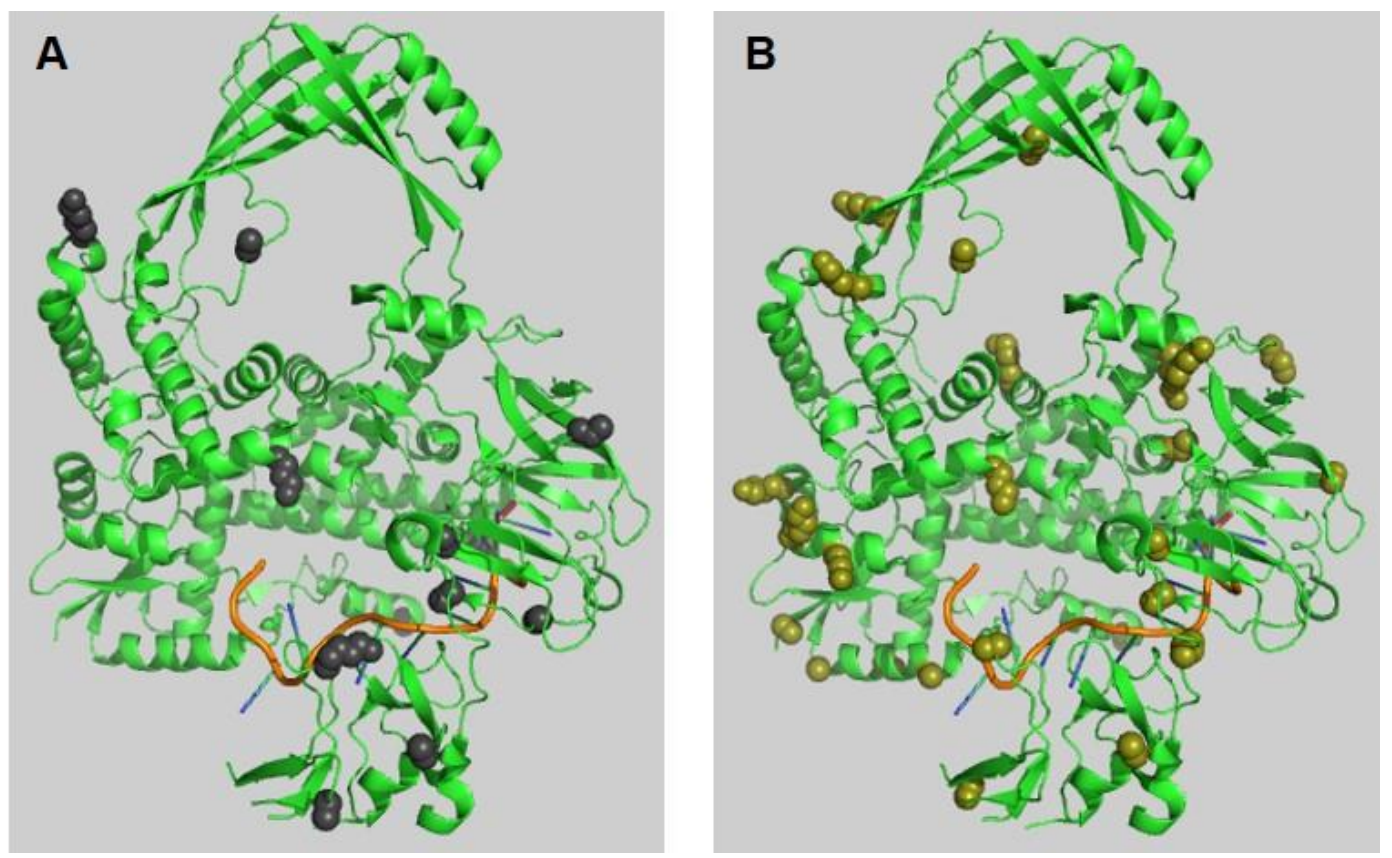

Figure 36 Positions of mass spectrometry identified acetylated lysine sites in acP acetylated TopA (A) Acetylated lysine in full length TopA from low concentration acP acetylation (PDB 4RUL). (B) Acetylated lysine in full length TopA from high concentration acP acetylation (PDB 4RUL). 


\section{Substitution of Lys-484 reduces TopA relaxation activity}

To model the effect of acetylation on individual lysine, site-directed mutagenesis to mutate lysine to alanine was performed. Alanine substitution of lysine removes the positive charge on lysine side chain mimicking the effect of lysine acetylation. Recombinant TopA mutant proteins were expressed in a $c o b B^{+}$genetic background, immunoblotting of wild-type TopA using anti acetyllysine antibody observed no acetylation signal (Figure $22 \mathrm{~A}$ lane 1).

The standard relaxation assays with negatively supercoiled plasmid DNA as the substrate showed that the relaxation activity of TopA K484A mutant was reduced by approximately 4-fold (Figure 37A). Meanwhile, assays on TopA K19A mutant, K144A

mutant, K153A mutant, K346 mutant, K788A mutant showed no significant reduction of TopA activity (Figure 37B). These results indicated that acetylation on lysine 484 may contribute to the reduction in TopA catalytic activity. 


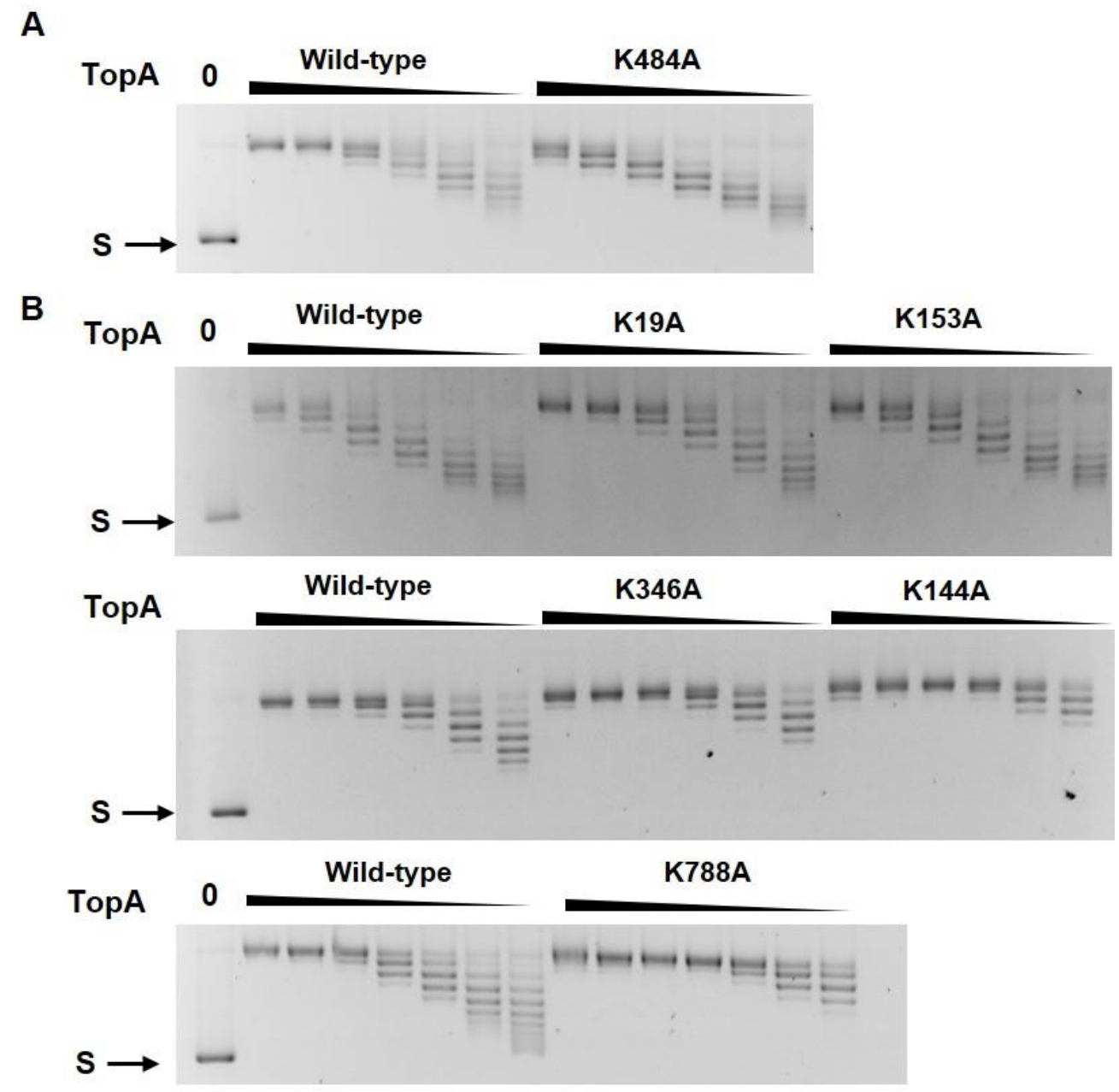

Figure 37 Relaxation assay comparing wild-type TopA with mutant TopA

(A) Wild-type TopA and K484A. Serial dilutions of TopA (50ng, 25ng, 12.5ng, 6ng, 3ng, 1.5ng) were incubated with $150 \mathrm{ng}$ negatively supercoiled plasmid DNA (S) at $37^{\circ} \mathrm{C}$ for $30 \mathrm{~min}$. (B) Wild-type TopA and K19A,K153A, K144A, K346A and K788A. Serial dilutions of K19A,K153A, K144A and K346A (50ng, $25 \mathrm{ng}, 12.5 \mathrm{ng}$, $6 \mathrm{ng}$, 3ng, $1.5 \mathrm{ng}$ ) were incubated with $150 \mathrm{ng}$ negatively supercoiled plasmid DNA (S) at $37^{\circ} \mathrm{C}$ for $30 \mathrm{~min}$. Serial dilutions of K788A (100ng, 50ng, 25ng, 12.5ng, 6ng, 3ng, 1.5ng) were incubated with 150 ng negatively supercoiled plasmid DNA at $37^{\circ} \mathrm{C}$ for $30 \mathrm{~min}$. 


\section{DISCUSSION}

In Chapter One, we have shown that lysine acetylation reduced TopA catalytic activity. In Chapter Two, we investigated physiological significance of TopA acetylation. To understand the molecular mechanism behind the activity reduction caused by acetylation, we modeled the effect of acetylation on individual lysine residues of TopA. We used site-directed mutagenesis to generate single mutation on selected lysine residues to alanine, mimicking the removal of positive charge from lysine by acetylation. Our results in Chapter three shed light on the potential effect of acetylation on individual lysine residue, and also provide insights on TopA relaxation mechanism proposed previously.

To select and mutate lysine residues which are both acetylated and significant, structural and mechanistic studies have been performed. As mentioned, global proteomics analysis reveal abundant acetylated lysine sites under various growth conditions (Baeza et al., 2014; Weinert et al., 2013; Weinert et al., 2017; Yu et al., 2008; Zhang et al., 2009a; Zhang et al., 2013a). Most of the acetylated lysines are located in the solvent exposed positions. They would be accessible to the CobB deacetylase as substrate, and may influence initial association between TopA and chromosomal DNA for the formation of the catalytically competent TopA-DNA complex. During TopA relaxation action, the interaction between TopA C-terminus and DNA is important for TopA to unwind DNA. The N-terminal domains of TopA can cleave and rejoin single-stranded DNA but cannot relax negatively supercoiled DNA due to the lack of C-terminal zinc-ribbon domains that are required for strand passage (Ahumada and Tse-Dinh, 2002; Beran-Steed and Tse-Dinh,

1989; Lima et al., 1993). In the recently obtained crystal structure of full length E. coli 
TopA, a single-stranded DNA is co-crystallized to bind to the C-terminal domains (PDB 4RUL) (Tan et al., 2015). Two potential binding models for interaction between C-terminal domains of TopA and ssDNA were generated. The first possible model is the single chain model, where TopA binds to G-strand and the torsional energy stored in the double helix drives TopA relaxation. The second one is the double chain model, where N-terminal domains of TopA bind to G-strand and C-terminal domains bind to T-strand. In this model, the $\mathrm{C}$-terminus guides strand passage. Our nuclease footprinting results support the double chain model (Figure 33, 34). TopA G116S was used to form the covalent complex with Bubble substrate. DNase I and MNase digestions showed the protection from TopA G116S on double-stranded regions and single-stranded regions of the substrate, which agrees with the double chain model. The results confirm the interaction between C-terminal domains and DNA during relaxation. Acetylation of lysines located on the C-terminal domains may have potential effect on T-strand binding and strand passage during relaxation. Lysine acetylation at the C-terminal domains of TopA may also influence its direct interaction with RNA polymerase during transcription elongation (Cheng et al., 2003). Moreover, this double strand model suggests that TopA might directly bind to the unwound DNA of the transcription bubble behind the transcription complex to relax the negative supercoils.

The acetylation states of some lysine residues on the N-terminal domains of TopA would also potentially affect the enzyme conformational change required for the opening and closing off the DNA cleavage gap for strand passage. Several lysine residues have been identified here to be proximal to T-strand during relaxation. TopA was crosslinked to the Substrate-Y with the reactive group PhSe attached to the 5' end of T-strand. TopA 
undergoes multiple conformational changes during relaxation (Viard and de la Tour, 2007). The crosslinking experiment help to identify interactions between TopA and DNA beyond the interactions shown by the available crystal structure. Out of 70 lysines in TopA, five lysines K19, K28, K279, K303 and K679 were crosslinked to the T-strand, suggesting these lysine residues were in close proximity to the $\mathrm{T}$-strand during catalysis. These lysines may be involved in the interaction between TopA and DNA in different steps during relaxation. K19, K28 and K679 located in D1 and D6 domains maybe involved in the initial binding of TopA to DNA prior to DNA cleavage (Figure 8, step 1). It is possible that acetylation on these three lysine sites may affect the initial binding of DNA by TopA. However, mutation of these individual lysine residue may only have a small effect on DNA binding and catalysis. During relaxation, the active site tyrosine cleaves DNA and forms a DNA gap to allow the T-strand to pass through. $\mathrm{K} 28$ together with $\mathrm{K} 303$ are positioned close to the active tyrosine, suggesting K28 and K303 may also involve in DNA cleavage and strand passage. Acetylation on K28 and K303 might change the efficiency of relaxation. K279 being close to a nucleotide-binding site in the central cavity, may be involved in the interaction with the T-strand DNA during and after strand passage (Figure 8, step 3). Acetylation on K279 might interfere the strand passage process.

To identify lysines that are acetylated by acetyl phosphate, mass spectrometry analysis of chemically acetylated TopA was performed. The hybrid quadrupole time-of flight mass spectrometry (QTOF), one of the major principle methods used in proteomic studies, provided us detailed information on acetylated lysine sites (Beck et al., 2015). 15 lysine residues have been identified to be acetylated under low acetyl phosphate 
concentration, while 44 lysine residues have been identified were acetylated under high acetyl phosphate concentration. More information could be obtained through the determination of the acetylation stoichiometry for these lysine residues in future studies. However, the broader distribution of acetylated lysine sites under high acetyl phosphate concentration is consistent with lower TopA catalytic activity shown in Figure 22.

Combining the lysine sites identified under physiological conditions and in vitro acetylated lysine sites identified by MS, with the consideration of the potential structural and mechanistic significance of these lysines, several lysine residues have been mutated and assayed for relaxation activity. Substitution on K484 have shown reduced activity about four-fold. Thus, acetylation on K484 may contribute to the reduction of TopA activity in cell extract (Figure 18), probably due to the location of K484 close to the catalytic center. Substitutions on other lysine residues (K19, K144, K153, K346, K788) showed similar relaxation activity as wild-type TopA, but may have impacts on proteinDNA or protein-protein interactions which need to be further addressed. 


\section{SUMMARY}

DNA topological states influence all major DNA transaction processes in living cells. DNA topoisomerases are essential for the maintenance of proper DNA topology for cell survival. Topoisomerases cleaves single-stranded or double-stranded DNA via a transesterification reaction. Based on the number of cleaved DNA strand, topoisomerases are classified as type I topoisomerase which cleaves one strand of DNA during reaction, and type II topoisomerase which cleaves two strands. Subfamilies of topoisomerases from type I and type II each have their distinct substrate specificities responsible to resolve certain types of topological problems raised during DNA transactions including DNA replication, transcription and recombination. Type IA topoisomerases are essential and ubiquitous enzymes that are present in all domain of life. Type IA topoisomerases including topoisomerase I and topoisomerase III in E. coli is important for removal of DNA supercoils.

$\mathrm{N}^{\varepsilon}$-lysine acetylation has been studied extensively for its role in eukaryotic cells. Recently, the investigation of lysine acetylation has been expanded to bacteria. $\mathrm{N}^{\varepsilon}$-lysine acetylation is a reversible post-translational modification which allows the protein to turn on and turn off its function. The donation of acetyl group from acetyl-phosphate and acetyl$\mathrm{CoA}$ is required for nonenzymatic and enzymatic acetylation in bacteria. Proteomic analysis revealed significant roles of acetylation in bacterial physiology for the regulation of central metabolism enzymes, motility, and chemotaxis gene expressions and stress resistance systems. Abundant proteins in E. coli are acetylated according to several proteomic reports. Although some E. coli proteins such as RNA polymerase, chemotaxis 
response regulator CheY and acetyl CoA synthetase have been well studied currently, the roles of acetylation on most $E$. coli proteins are largely unexplored. Among these acetylated proteins, DNA topoisomerase I has been shown to be acetylated at multiple lysine residues implying the potential regulatory role of acetylation on E. coli TopA.

Topoisomerase I (TopA) is a vital enzyme for cell survival. This enzyme is highly efficient for binding to single-stranded DNA region and relaxation of negative supercoiling. Therefore, TopA is well suited to resolve the excess negative supercoiling generated behind RNA polymerase during DNA transcription and in suppression of R-loop accumulation. The transcription of topA gene is increased with DNA negative supercoiling level. During stress challenges including heat shock and oxidative stress, the relaxation of transcription driven negative supercoiling by TopA is important for regulation of DNA supercoiling to fit the need for cell survival in response to these environmental challenge. Considering the significant role of TopA, we hypothesized that acetylation state of TopA is important for bacterial physiology. The overall goal of this dissertation project is to clarify the effect of topoisomerase I acetylation in E. coli at both molecular and physiological level, as well as possible mechanisms related to reduction of TopA relaxation activity following acetylation.

I hypothesized that acetylation inhibits TopA activity. In chapter one, the effect of TopA acetylation was studied using $\Delta \operatorname{cobB}$ mutant $E$. coli strain. TopA relaxation activity in the $\Delta c o b B$ mutant cell extract was compared to that in the wild-type cell extract. I found that TopA activity in the total cell lysate of $\triangle \operatorname{cobB}$ mutant strain was significantly reduced even though topA gene transcription and protein expression levels were increased about 1.5-fold. The change of TopA expression in the absence of CobB is consistent with the 
increased DNA supercoiling level observed in chapter two. On the basis of these results, we proposed a homeostatic regulation model of TopA acetylation that the reduction of TopA relaxation activity due to the missing deacetylase function in the $\Delta c o b B$ mutant increases DNA negative supercoiling, which in turn upregulates topA gene transcription and TopA expression. Evidence showing TopA activity reduction in the in vitro acPmediated nonenzymatic lysine acetylation confirmed our hypothesis that acetylation inhibits TopA activity. Besides the global effect of TopA acetylation on DNA supercoiling, we have showed that TopA acetylation affect cell growth. The $\triangle c o b B$ mutant displayed a slow growth phenotype in entering the exponential phase potentially due to the global effect of acetylation on a large number of $E$. coli proteins. Intriguingly, the slow growth phenotype of $\triangle \operatorname{cobB}$ mutant is reversed by overexpression of recombinant TopA. This could because of the global effect of TopA in regulation of DNA supercoiling. The reverse effect of slow growth by TopA overexpression is partial, suggesting other cellular processes which are not DNA supercoiling related are involved. Analysis of TopA acetylation showed that the acetylation level of TopA is associated with growth stages. TopA was found to be at its lowest acetylation level during growth transition into stationary phase from exponential phase, and at its highest acetylation during growth arrest. The correlation between degree of lysine acetylation and TopA activity was further established using purified His-tagged TopA expressed from the chromosome.

More importantly, we found that deacetylation of TopA by CobB could partially reverse the effects of TopA acetylation. Sirtuin deacetylase CobB, the only well studied deacetylase so far in E. coli, was able to prevent the TopA inactivation in the in vitro assay. 
Introduction of plasmid $\mathrm{pCobB}$ into the $\triangle \operatorname{cob} B$ mutant also rescued the excess negative DNA supercoiling and the delayed cell growth. In a previous report, TopA is identified as a CobB interacting partner. Here, we confirmed the TopA and CobB interaction by pulldown assay using purified TopA and CobB. Hence, we conclude that acetylation on TopA reduces the enzyme activity, and the deacetylation of TopA by CobB is of physiological significance.

We further analyze the potential mechanism of reduction in TopA relaxation activity following acetylation. Potential acetylated lysine residues of TopA were first identified based on previous proteomics reports, 20 out of 70 lysine residues were found to be acetylated. Most of the acetylated lysine residues are prone to locate in the solvent exposed positions which are accessible for CobB deacetylase. Acetylation on these exposed lysines may influence association between TopA and its DNA substrate required during the enzyme relaxation process. We conducted a structural examination of TopA to further identify potentially significant lysine acetylation sites. Nuclease footprinting and oxidative crosslinking were used to elucidate TopA-DNA interaction and DNA strand passage. The nuclease footprinting experiments conducted following the recent determination of a co-crystal structure of TopA-ssDNA complex revealed that T-strand DNA is bound to C-terminal domains of TopA while G-strand DNA is bound to N-terminal domains. This double chain interaction model is in agreement with the previous finding that N-terminal domains of TopA alone can cleave and rejoin DNA but cannot relax negative supercoils. T-strand with a reactive group PhSe attached to the 5' end was used to crosslinked with TopA on amino acids which are in close proximity during relaxation. 
During TopA relaxation, five lysine residues (Lys-19, Lys-28, Lys-279, Lys-303 and Lys649) were identified as crosslinking sites and may potentially affect the enzyme conformational change or strand passage during catalysis. These five lysine residues are either acetylation sites identified by previous proteomics studies or close to acetylation sites. As showed in chapter one, the relaxation activity of TopA acetylated by the in vitro acP mediated acetylation is significantly reduced. In chapter three, we analyzed acetylated lysine residues of in vitro acetylated TopA using mass spectrometry. Abundant lysine acetylation sites have been identified. These TopA structural analysis and identified acetylation sites provide information for studying the role of individual lysine acetylation. We started with genetic mimic of acetylation on Lys-19, Lys-144, Lys-153, Lys-346, Lys484 and Lys-788. We found alanine substitution of Lys-484 resulted in reduced relaxation activity. According to the crystal structure of TopA, Lys-484 is positioned at the edge of cavity hole, which is close to the catalytic center. Acetylation of Lys-484 might affect TopA-DNA interaction or TopA conformations required for catalysis. Although alanine substitution of other lysine residues showed no activity reduction, the interactions of TopA with other protein may be affected. It may also be necessary to combine the effect of multiple lysine modification to observe the effect on TopA relaxation activity. The elucidation of the biochemical significance of individual TopA lysine acetylation sites would require future mechanistic and genetic studies.

The results presented in this project support modulation of TopA catalytic activity via lysine acetylation, and a physiological function of E. coli CobB in preventing TopA inactivation from excess lysine acetylation. There are questions that remain to be answered 
with regard to potential regulation of topoisomerase activity and DNA supercoiling as a global signal by acetylation-deacetylation in bacteria. Determination of the acetylation stoichiometry at specific TopA lysine residues under various growth conditions is currently incomplete. In addition to the confirmed lysine acetyltransferase YfiQ, there may be other E. coli acetyltransferases that remain to be identified and characterized. Lysine acetylationdeacetylation has been linked to stress response in bacteria, and may provide a more rapid mechanism for modification of TopA catalytic activity than the transcription response from topA promoters. 


\section{REFERENCES}

AbouElfetouh, A., Kuhn, M.L., Hu, L.I., Scholle, M.D., Sorensen, D.J., Sahu, A.K., Becher, D., Antelmann, H., Mrksich, M., Anderson, W.F., et al. (2015). The E. coli sirtuin CobB shows no preference for enzymatic and nonenzymatic lysine acetylation substrate sites. MicrobiologyOpen 4, 66-83.

Ahumada, A., and Tse-Dinh, Y.C. (1998). The Zn(II) binding motifs of E. coli DNA topoisomerase I is part of a high-affinity DNA binding domain. Biochem Biophys Res Commun 251, 509-514.

Ahumada, A., and Tse-Dinh, Y.C. (2002). The role of the $\mathrm{Zn}$ (II) binding domain in the mechanism of E. coli DNA topoisomerase I. BMC biochemistry 3, 13.

Aksnes, H., Hole, K., and Arnesen, T. (2015). Molecular, cellular, and physiological significance of N-terminal acetylation. Int Rev Cell Mol Biol 316, 267-305.

Allfrey, V.G., Faulkner, R., and Mirsky, A.E. (1964). Acetylation and methylation of histones and their possible role in the regulation of RNA synthesis. Proc Natl Acad Sci U S A 51, 786-794.

Allfrey, V.G., and Mirsky, A.E. (1964). Structural modifications of histones and their possible role in the regulation of RNA Synthesis. Science 144, 559.

Andersson, D.I., and Hughes, D. (2014). Microbiological effects of sublethal levels of antibiotics. Nat Rev Microbiol 12, 465-478.

Aravind, L., Leipe, D.D., and Koonin, E.V. (1998). Toprim--a conserved catalytic domain in type IA and II topoisomerases, DnaG-type primases, OLD family nucleases and RecR proteins. Nucleic Acids Res 26, 4205-4213.

Arnesen, T., Van Damme, P., Polevoda, B., Helsens, K., Evjenth, R., Colaert, N., Varhaug, J.E., Vandekerckhove, J., Lillehaug, J.R., Sherman, F., et al. (2009). Proteomics analyses reveal the evolutionary conservation and divergence of $\mathrm{N}$-terminal acetyltransferases from yeast and humans. Proc Natl Acad Sci U S A 106, 8157-8162.

Baeza, J., Dowell, J.A., Smallegan, M.J., Fan, J., Amador-Noguez, D., Khan, Z., and Denu, J.M. (2014). Stoichiometry of site-specific lysine acetylation in an entire proteome. J Biol Chem 289, 21326-21338.

Baeza, J., Smallegan, M.J., and Denu, J.M. (2015). Site-specific reactivity of nonenzymatic lysine acetylation. ACS Chem Biol 10, 122-128.

Baker, N.M., Rajan, R., and Mondragon, A. (2009). Structural studies of type I topoisomerases. Nucleic Acids Res 37, 693-701. 
Balke, V.L., and Gralla, J.D. (1987). Changes in the linking number of supercoiled DNA accompany growth transitions in Escherichia coli. J Bacteriol 169, 4499-4506.

Banda, S., Tiwari, P.B., Darici, Y., and Tse-Dinh, Y.C. (2016). Investigating direct interaction between Escherichia coli topoisomerase I and RecA. Gene 585, 65-70.

Barak, R., and Eisenbach, M. (2001). Acetylation of the response regulator, CheY, is involved in bacterial chemotaxis. Mol Microbiol 40, 731-743.

Barak, R., and Eisenbach, M. (2004). Co-regulation of acetylation and phosphorylation of CheY, a response regulator in chemotaxis of Escherichia coli. J Mol Biol 342, 375-381.

Barak, R., Prasad, K., Shainskaya, A., Wolfe, A.J., and Eisenbach, M. (2004). Acetylation of the chemotaxis response regulator $\mathrm{CheY}$ by acetyl-CoA synthetase purified from Escherichia coli. J Mol Biol 342, 383-401.

Baranello, L., Levens, D., Gupta, A., and Kouzine, F. (2012). The importance of being supercoiled: how DNA mechanics regulate dynamic processes. Biochim Biophys Acta $1819,632-638$.

Baron, S., Afanzar, O., and Eisenbach, M. (2017). Methylation-independent adaptation in chemotaxis of Escherichia coli involves acetylation-dependent speed adaptation. FEBS letters 591, 331-337.

Baxter, J., and Diffley, J.F. (2008). Topoisomerase II inactivation prevents the completion of DNA replication in budding yeast. Mol Cell 30, 790-802.

Beck, S., Michalski, A., Raether, O., Lubeck, M., Kaspar, S., Goedecke, N., Baessmann, C., Hornburg, D., Meier, F., Paron, I., et al. (2015). The Impact II, a very high-resolution quadrupole time-of-flight instrument (QTOF) for deep shotgun proteomics. Mol Cell Proteomics 14, 2014-2029.

Been, M.D., and Champoux, J.J. (1980). Breakage of single-stranded DNA by rat liver nicking-closing enzyme with the formation of a DNA-enzyme complex. Nucleic Acids Res $8,6129-6142$.

Beran-Steed, R.K., and Tse-Dinh, Y.C. (1989). The carboxyl terminal domain of Escherichia coli DNA topoisomerase I confers higher affinity to DNA. Proteins 6, 249258.

Bergerat, A., Gadelle, D., and Forterre, P. (1994). Purification of a DNA topoisomerase II from the hyperthermophilic archaeon Sulfolobus shibatae. A thermostable enzyme with both bacterial and eucaryal features. J Biol Chem 269, 27663-27669.

Bernal, V., Castano-Cerezo, S., Gallego-Jara, J., Ecija-Conesa, A., de Diego, T., Iborra, J.L., and Canovas, M. (2014). Regulation of bacterial physiology by lysine acetylation of proteins. N Biotechnol 31, 586-595. 
Cain, J.A., Solis, N., and Cordwell, S.J. (2014). Beyond gene expression: the impact of protein post-translational modifications in bacteria. J Proteomics 97, 265-286.

Carabetta, V.J., and Cristea, I.M. (2017). The regulation, function, and detection of protein acetylation in bacteria. J Bacteriol.

Castano-Cerezo, S., Bernal, V., Post, H., Fuhrer, T., Cappadona, S., Sanchez-Diaz, N.C., Sauer, U., Heck, A.J., Altelaar, A.F., and Canovas, M. (2014). Protein acetylation affects acetate metabolism, motility and acid stress response in Escherichia coli. Mol Syst Biol. 10,762 .

Cejka, P., Cannavo, E., Polaczek, P., Masuda-Sasa, T., Pokharel, S., Campbell, J.L., and Kowalczykowski, S.C. (2010). DNA end resection by Dna2-Sgs1-RPA and its stimulation by Top3-Rmi1 and Mre11-Rad50-Xrs2. Nature 467, 112-116.

Champoux, J.J. (2001). DNA topoisomerases: structure, function, and mechanism. Annu Rev Biochem 70, 369-413.

Champoux, J.J., and Dulbecco, R. (1972). An activity from mammalian cells that untwists superhelical DNA-a possible swivel for DNA replication (polyoma-ethidium bromidemouse-embryo cells-dye binding assay). Proc Natl Acad Sci U S A 69, 143-146.

Charbaut, E., Redeker, V., Rossier, J., and Sobel, A. (2002). N-terminal acetylation of ectopic recombinant proteins in Escherichia coli. FEBS letters 529, 341-345.

Chen, S.H., Chan, N.L., and Hsieh, T.S. (2013). New mechanistic and functional insights into DNA topoisomerases. Annu Rev Biochem 82, 139-170.

Chen, S.J., and Wang, J.C. (1998). Identification of active site residues in Escherichia coli DNA topoisomerase I. J Biol Chem 273, 6050-6056.

Cheng, B., Shukla, S., Vasunilashorn, S., Mukhopadhyay, S., and Tse-Dinh, Y.C. (2005). Bacterial cell killing mediated by topoisomerase I DNA cleavage activity. J Biol Chem 280, 38489-38495.

Cheng, B., Zhou, Q., Weng, L., Leszyk, J.D., Greenberg, M.M., and Tse-Dinh, Y.C. (2017). Identification of proximal sites for unwound DNA substrate in Escherichia coli topoisomerase I with oxidative crosslinking. FEBS letters 591, 28-38.

Cheng, B., Zhu, C.X., Ji, C., Ahumada, A., and Tse-Dinh, Y.C. (2003). Direct interaction between Escherichia coli RNA polymerase and the zinc ribbon domains of DNA topoisomerase I. J Biol Chem 278, 30705-30710.

Cheung, K.J., Badarinarayana, V., Selinger, D.W., Janse, D., and Church, G.M. (2003). A microarray-based antibiotic screen identifies a regulatory role for supercoiling in the osmotic stress response of Escherichia coli. Genome Res 13, 206-215. 
Choudhary, C., Kumar, C., Gnad, F., Nielsen, M.L., Rehman, M., Walther, T.C., Olsen, J.V., and Mann, M. (2009). Lysine acetylation targets protein complexes and co-regulates major cellular functions. Science 325, 834-840.

Corbett, K.D., and Berger, J.M. (2003). Emerging roles for plant topoisomerase VI. Chem Biol 10, 107-111.

Corbett, K.D., and Berger, J.M. (2004). Structure, molecular mechanisms, and evolutionary relationships in DNA topoisomerases. Annu Rev Biophys Biomol Struct 33, 95-118.

Crosby, H.A., Heiniger, E.K., Harwood, C.S., and Escalante-Semerena, J.C. (2010). Reversible $\mathrm{N}$ epsilon-lysine acetylation regulates the activity of acyl-CoA synthetases involved in anaerobic benzoate catabolism in Rhodopseudomonas palustris. Mol Microbiol $76,874-888$.

Da Re, S., Garnier, F., Guerin, E., Campoy, S., Denis, F., and Ploy, M.C. (2009). The SOS response promotes qnrB quinolone-resistance determinant expression. EMBO reports 10, 929-933.

Datsenko, K.A., and Wanner, B.L. (2000). One-step inactivation of chromosomal genes in Escherichia coli K-12 using PCR products. Proc Natl Acad Sci U S A 97, 6640-6645.

Davis, M.C., Kesthely, C.A., Franklin, E.A., and MacLellan, S.R. (2017). The essential activities of the bacterial sigma factor. Can J Microbiol 63, 89-99.

DiGate, R.J., and Marians, K.J. (1988). Identification of a potent decatenating enzyme from Escherichia coli. J Biol Chem 263, 13366-13373.

DiNardo, S., Voelkel, K.A., Sternglanz, R., Reynolds, A.E., and Wright, A. (1982). Escherichia coli DNA topoisomerase I mutants have compensatory mutations in DNA gyrase genes. Cell 31, 43-51.

Dixon, J.R., Selvaraj, S., Yue, F., Kim, A., Li, Y., Shen, Y., Hu, M., Liu, J.S., and Ren, B. (2012). Topological domains in mammalian genomes identified by analysis of chromatin interactions. Nature 485, 376-380.

Doyle, S.A. (2005). High-throughput cloning for proteomics research. Methods Mol Biol $310,107-113$

Drazic, A., Myklebust, L.M., Ree, R., and Arnesen, T. (2016). The world of protein acetylation. Biochim Biophys Acta 1864, 1372-1401.

Drlica, K. (1992). Control of bacterial DNA supercoiling. Mol Microbiol 6, 425-433.

Drlica, K., and Malik, M. (2003). Fluoroquinolones: action and resistance. Curr Top Med Chem 3, 249-282. 
Drolet, M. (2006). Growth inhibition mediated by excess negative supercoiling: the interplay between transcription elongation, R-loop formation and DNA topology. Mol Microbiol 59, 723-730.

Drolet, M., Bi, X., and Liu, L.F. (1994). Hypernegative supercoiling of the DNA template during transcription elongation in vitro. J Biol Chem 269, 2068-2074.

Drolet, M., Phoenix, P., Menzel, R., Masse, E., Liu, L.F., and Crouch, R.J. (1995). Overexpression of RNase H partially complements the growth defect of an Escherichia coli delta topA mutant: R-loop formation is a major problem in the absence of DNA topoisomerase I. Proc Natl Acad Sci U S A 92, 3526-3530.

Eberharter, A., and Becker, P.B. (2002). Histone acetylation: a switch between repressive and permissive chromatin. Second in review series on chromatin dynamics. EMBO reports $3,224-229$.

Fan, J., Krautkramer, K.A., Feldman, J.L., and Denu, J.M. (2015). Metabolic regulation of histone post-translational modifications. ACS Chem Biol 10, 95-108.

Feinberg, H., Changela, A., and Mondragon, A. (1999). Protein-nucleotide interactions in E. coli DNA topoisomerase I. Nat Struct Biol 6, 961-968.

Filippakopoulos, P., and Knapp, S. (2014). Targeting bromodomains: epigenetic readers of lysine acetylation. Nat Rev Drug Discov 13, 337-356.

Forterre, P., Gribaldo, S., Gadelle, D., and Serre, M.C. (2007). Origin and evolution of DNA topoisomerases. Biochimie 89, 427-446.

Fraiberg, M., Afanzar, O., Cassidy, C.K., Gabashvili, A., Schulten, K., Levin, Y., and Eisenbach, M. (2015). CheY's acetylation sites responsible for generating clockwise flagellar rotation in Escherichia coli. Mol Microbiol 95, 231-244.

Fredericks, C.E., Shibata, S., Aizawa, S., Reimann, S.A., and Wolfe, A.J. (2006). Acetyl phosphate-sensitive regulation of flagellar biogenesis and capsular biosynthesis depends on the Rcs phosphorelay. Mol Microbiol 61, 734-747.

Freeman, L.A., and Garrard, W.T. (1992). DNA supercoiling in chromatin structure and gene expression. Crit Rev Eukaryot Gene Expr 2, 165-209.

Fukuda, T., Kugou, K., Sasanuma, H., Shibata, T., and Ohta, K. (2008). Targeted induction of meiotic double-strand breaks reveals chromosomal domain-dependent regulation of Spo11 and interactions among potential sites of meiotic recombination. Nucleic Acids Res 36, 984-997.

Gadelle, D., Filee, J., Buhler, C., and Forterre, P. (2003). Phylogenomics of type II DNA topoisomerases. Bioessays 25, 232-242. 
Gardner, J.G., and Escalante-Semerena, J.C. (2009). In Bacillus subtilis, the sirtuin protein deacetylase, encoded by the srtN gene (formerly yhdZ), and functions encoded by the acuABC genes control the activity of acetyl coenzyme A synthetase. J Bacteriol 191, 17491755.

Gardner, J.G., Grundy, F.J., Henkin, T.M., and Escalante-Semerena, J.C. (2006). Control of acetyl-coenzyme A synthetase (AcsA) activity by acetylation/deacetylation without NAD $(+)$ involvement in Bacillus subtilis. J Bacteriol 188, 5460-5468.

Gellert, M., Mizuuchi, K., O'Dea, M.H., and Nash, H.A. (1976). DNA gyrase: an enzyme that introduces superhelical turns into DNA. Proc Natl Acad Sci U S A 73, 3872-3876.

Gershey, E.L., Vidali, G., and Allfrey, V.G. (1968). Chemical studies of histone acetylation. The occurrence of epsilon-N-acetyllysine in the f2a1 histone. J Biol Chem 243, 5018-5022.

Ghosh, S., Padmanabhan, B., Anand, C., and Nagaraja, V. (2016). Lysine acetylation of the Mycobacterium tuberculosis HU protein modulates its DNA binding and genome organization. Mol Microbiol 100, 577-588.

Giglione, C., Vallon, O., and Meinnel, T. (2003). Control of protein life-span by N-terminal methionine excision. The EMBO journal 22, 13-23.

Gilbert, N., and Allan, J. (2014). Supercoiling in DNA and chromatin. Curr Opin Genet Dev 25, 15-21.

Giles, G.I., and Sharma, R.P. (2005). Topoisomerase enzymes as therapeutic targets for cancer chemotherapy. Med Chem 1, 383-394.

Goldstein, E., and Drlica, K. (1984). Regulation of bacterial DNA supercoiling: plasmid linking numbers vary with growth temperature. Proc Natl Acad Sci U S A 81, 4046-4050.

Gordiyenko, Y., Deroo, S., Zhou, M., Videler, H., and Robinson, C.V. (2008). Acetylation of L12 increases interactions in the Escherichia coli ribosomal stalk complex. J Mol Biol $380,404-414$.

Gray, M.W., Burger, G., and Lang, B.F. (1999). Mitochondrial evolution. Science 283, 1476-1481.

Griffiths AJF, G.W., Miller JH, et al. (1999). Modern Genetic Analysis. New York: W. H. Freeman; The Nature of Genomes.

Gu, W., and Roeder, R.G. (1997). Activation of p53 sequence-specific DNA binding by acetylation of the p53 C-terminal domain. Cell 90, 595-606.

Guzman, L.M., Belin, D., Carson, M.J., and Beckwith, J. (1995). Tight regulation, modulation, and high-level expression by vectors containing the arabinose PBAD promoter. J Bacteriol 177, 4121-4130. 
Hallows, W.C., Lee, S., and Denu, J.M. (2006). Sirtuins deacetylate and activate mammalian acetyl-CoA synthetases. Proc Natl Acad Sci U S A 103, 10230-10235.

Hamperl, S., and Cimprich, K.A. (2014). The contribution of co-transcriptional RNA:DNA hybrid structures to DNA damage and genome instability. DNA Repair (Amst) 19, 84-94.

Harbison, C.T., Gordon, D.B., Lee, T.I., Rinaldi, N.J., Macisaac, K.D., Danford, T.W., Hannett, N.M., Tagne, J.B., Reynolds, D.B., Yoo, J., et al. (2004). Transcriptional regulatory code of a eukaryotic genome. Nature 431, 99-104.

Harmon, F.G., Brockman, J.P., and Kowalczykowski, S.C. (2003). RecQ helicase stimulates both DNA catenation and changes in DNA topology by topoisomerase III. J Biol Chem 278, 42668-42678.

Hartung, F., and Puchta, H. (2001). Molecular characterization of homologues of both subunits A (SPO11) and B of the archaebacterial topoisomerase 6 in plants. Gene 271, 8186.

Helsens, K., Van Damme, P., Degroeve, S., Martens, L., Arnesen, T., Vandekerckhove, J., and Gevaert, K. (2011). Bioinformatics analysis of a Saccharomyces cerevisiae N-terminal proteome provides evidence of alternative translation initiation and post-translational Nterminal acetylation. J Proteome Res 10, 3578-3589.

Hentchel, K.L., and Escalante-Semerena, J.C. (2015). Acylation of biomolecules in prokaryotes: a widespread strategy for the control of biological function and metabolic stress. Microbiol Mol Biol Rev 79, 321-346.

Hiasa, H., DiGate, R.J., and Marians, K.J. (1994). Decatenating activity of Escherichia coli DNA gyrase and topoisomerases I and III during oriC and pBR322 DNA replication in vitro. J Biol Chem 269, 2093-2099.

Holm, C., Goto, T., Wang, J.C., and Botstein, D. (1985). DNA topoisomerase II is required at the time of mitosis in yeast. Cell 41, 553-563.

Hosp, F., Lassowskat, I., Santoro, V., De Vleesschauwer, D., Fliegner, D., Redestig, H., Mann, M., Christian, S., Hannah, M.A., and Finkemeier, I. (2017). Lysine acetylation in mitochondria: From inventory to function. Mitochondrion 33, 58-71.

Hsieh, L.S., Burger, R.M., and Drlica, K. (1991). Bacterial DNA supercoiling and [ATP]/[ADP]. Changes associated with a transition to anaerobic growth. J Mol Biol 219, 443-450.

Hu, L.I., Lima, B.P., and Wolfe, A.J. (2010). Bacterial protein acetylation: the dawning of a new age. Mol Microbiol 77, 15-21.

Janion, C. (2008). Inducible SOS response system of DNA repair and mutagenesis in Escherichia coli. Int J Biol Sci 4, 338-344. 
Kaguni, J.M., and Kornberg, A. (1984). Replication initiated at the origin (oriC) of the $E$. coli chromosome reconstituted with purified enzymes. Cell 38, 183-190.

Kang, K., Choi, J.M., Fox, J.M., Snyder, P.W., Moustakas, D.T., and Whitesides, G.M. (2016). Acetylation of Surface Lysine Groups of a Protein Alters the Organization and Composition of Its Crystal Contacts. J Phys Chem B 120, 6461-6468.

Karve, T.M., and Cheema, A.K. (2011). Small changes huge impact: the role of protein posttranslational modifications in cellular homeostasis and disease. J Amino Acids 2011, 207691.

Keszthelyi, A., Minchell, N.E., and Baxter, J. (2016). The Causes and Consequences of Topological Stress during DNA Replication. Genes (Basel) 7.

Kim, D., Yu, B.J., Kim, J.A., Lee, Y.J., Choi, S.G., Kang, S., and Pan, J.G. (2013). The acetylproteome of Gram-positive model bacterium Bacillus subtilis. Proteomics 13, 17261736.

Kim, S.C., Sprung, R., Chen, Y., Xu, Y., Ball, H., Pei, J., Cheng, T., Kho, Y., Xiao, H., Xiao, L., et al. (2006). Substrate and functional diversity of lysine acetylation revealed by a proteomics survey. Mol Cell 23, 607-618.

Kitagawa, M., Ara, T., Arifuzzaman, M., Ioka-Nakamichi, T., Inamoto, E., Toyonaga, H., and Mori, H. (2005). Complete set of ORF clones of Escherichia coli ASKA library (a complete set of $E$. coli K-12 ORF archive): unique resources for biological research. DNA Res 12, 291-299.

Klein, A.H., Shulla, A., Reimann, S.A., Keating, D.H., and Wolfe, A.J. (2007). The intracellular concentration of acetyl phosphate in Escherichia coli is sufficient for direct phosphorylation of two-component response regulators. J Bacteriol 189, 5574-5581.

Knorre, D.G., Kudryashova, N.V., and Godovikova, T.S. (2009). Chemical and functional aspects of posttranslational modification of proteins. Acta naturae 1, 29-51.

Kuhn, M.L., Zemaitaitis, B., Hu, L.I., Sahu, A., Sorensen, D., Minasov, G., Lima, B.P., Scholle, M., Mrksich, M., Anderson, W.F., et al. (2014). Structural, kinetic and proteomic characterization of acetyl phosphate-dependent bacterial protein acetylation. PloS one 9, e94816.

Lesley, S.A., Jovanovich, S.B., Tse-Dinh, Y.C., and Burgess, R.R. (1990). Identification of a heat shock promoter in the topA gene of Escherichia coli. J Bacteriol 172, 6871-6874.

Li, R., Chen, P., Gu, J., and Deng, J.Y. (2013). Acetylation reduces the ability of CheY to undergo autophosphorylation. FEMS Microbiol Lett 347, 70-76. 
Li, R., Gu, J., Chen, Y.Y., Xiao, C.L., Wang, L.W., Zhang, Z.P., Bi, L.J., Wei, H.P., Wang, X.D., Deng, J.Y., et al. (2010). CobB regulates Escherichia coli chemotaxis by deacetylating the response regulator CheY. Mol Microbiol 76, 1162-1174.

Li, Z., Mondragon, A., Hiasa, H., Marians, K.J., and DiGate, R.J. (2000). Identification of a unique domain essential for Escherichia coli DNA topoisomerase III-catalysed decatenation of replication intermediates. Mol Microbiol 35, 888-895.

Liang, W., and Deutscher, M.P. (2012). Post-translational modification of RNase R is regulated by stress-dependent reduction in the acetylating enzyme Pka (YfiQ). RNA 18, $37-41$.

Liang, W., Malhotra, A., and Deutscher, M.P. (2011). Acetylation regulates the stability of a bacterial protein: growth stage-dependent modification of RNase R. Mol Cell 44, 160166.

Liao, G., Xie, L., Li, X., Cheng, Z., and Xie, J. (2014). Unexpected extensive lysine acetylation in the trump-card antibiotic producer Streptomyces roseosporus revealed by proteome-wide profiling. J Proteomics 106, 260-269.

Liarzi, O., Barak, R., Bronner, V., Dines, M., Sagi, Y., Shainskaya, A., and Eisenbach, M. (2010). Acetylation represses the binding of CheY to its target proteins. Mol Microbiol 76, 932-943.

Lima, B.P., Antelmann, H., Gronau, K., Chi, B.K., Becher, D., Brinsmade, S.R., and Wolfe, A.J. (2011). Involvement of protein acetylation in glucose-induced transcription of a stressresponsive promoter. Mol Microbiol 81, 1190-1204.

Lima, B.P., Thanh Huyen, T.T., Basell, K., Becher, D., Antelmann, H., and Wolfe, A.J. (2012). Inhibition of acetyl phosphate-dependent transcription by an acetylatable lysine on RNA polymerase. J Biol Chem 287, 32147-32160.

Lima, C.D., Wang, J.C., and Mondragon, A. (1993). Crystallization of a $67 \mathrm{kDa}$ fragment of Escherichia coli DNA topoisomerase I. J Mol Biol 232, 1213-1216.

Lima, C.D., Wang, J.C., and Mondragon, A. (1994). Three-dimensional structure of the 67K N-terminal fragment of E. coli DNA topoisomerase I. Nature 367, 138-146.

Liu, C.X., Wu, F.L., Jiang, H.W., He, X., Guo, S.J., and Tao, S.C. (2014). Global identification of CobB interactors by an Escherichia coli proteome microarray. Acta Biochim Biophys Sin 46, 548-555.

Liu, I.F., Sutherland, J.H., Cheng, B., and Tse-Dinh, Y.C. (2011). Topoisomerase I function during Escherichia coli response to antibiotics and stress enhances cell killing from stabilization of its cleavage complex. J Antimicrob Chemother 66, 1518-1524. 
Liu, L.F., and Wang, J.C. (1987). Supercoiling of the DNA template during transcription. Proc Natl Acad Sci U S A 84, 7024-7027.

Ma, Q., and Wood, T.K. (2011). Protein acetylation in prokaryotes increases stress resistance. Biochem Biophys Res Commun 410, 846-851.

Malik, M., Bensaid, A., Rouviere-Yaniv, J., and Drlica, K. (1996). Histone-like protein $\mathrm{HU}$ and bacterial DNA topology: suppression of an HU deficiency by gyrase mutations. J Mol Biol 256, 66-76.

Mao, Y., Desai, S.D., Ting, C.Y., Hwang, J., and Liu, L.F. (2001). 26 S proteasomemediated degradation of topoisomerase II cleavable complexes. J Biol Chem 276, 4065240658.

Mao, Y., Sun, M., Desai, S.D., and Liu, L.F. (2000). SUMO-1 conjugation to topoisomerase I: A possible repair response to topoisomerase-mediated DNA damage. Proc Natl Acad Sci U S A 97, 4046-4051.

Masse, E., and Drolet, M. (1999a). Escherichia coli DNA topoisomerase I inhibits R-loop formation by relaxing transcription-induced negative supercoiling. J Biol Chem 274, $16659-16664$.

Masse, E., and Drolet, M. (1999b). Relaxation of transcription-induced negative supercoiling is an essential function of Escherichia coli DNA topoisomerase I. J Biol Chem $274,16654-16658$.

Masse, E., Phoenix, P., and Drolet, M. (1997). DNA topoisomerases regulate R-loop formation during transcription of the rrnB operon in Escherichia coli. J Biol Chem 272, $12816-12823$.

Menzel, R., and Gellert, M. (1983). Regulation of the genes for E. coli DNA gyrase: homeostatic control of DNA supercoiling. Cell 34, 105-113.

Miao, L., Fang, H., Li, Y., and Chen, H. (2007). Studies of the in vitro Nalphaacetyltransferase activities of E. coli RimL protein. Biochem Biophys Res Commun 357, 641-647.

Mirkin, S.M. (2001). DNA Topology: Fundamentals. eLS. .

Mondragon, A., and DiGate, R. (1999). The structure of Escherichia coli DNA topoisomerase III. Structure 7, 1373-1383.

Narula, G., Annamalai, T., Aedo, S., Cheng, B., Sorokin, E., Wong, A., and Tse-Dinh, Y.C. (2011). The strictly conserved Arg-321 residue in the active site of Escherichia coli topoisomerase I plays a critical role in DNA rejoining. J Biol Chem 286, 18673-18680. 
Narula, G., and Tse-Dinh, Y.C. (2012). Residues of E. coli topoisomerase I conserved for interaction with a specific cytosine base to facilitate DNA cleavage. Nucleic Acids Res 40, 9233-9243.

Nichols, M.D., DeAngelis, K., Keck, J.L., and Berger, J.M. (1999). Structure and function of an archaeal topoisomerase VI subunit with homology to the meiotic recombination factor Spo11. The EMBO journal 18, 6177-6188.

Ogata, Y., Inoue, R., Mizushima, T., Kano, Y., Miki, T., and Sekimizu, K. (1997). Heat shock-induced excessive relaxation of DNA in Escherichia coli mutants lacking the histone-like protein HU. Biochim Biophys Acta 1353, 298-306.

Ogata, Y., Mizushima, T., Kataoka, K., Kita, K., Miki, T., and Sekimizu, K. (1996). DnaK heat shock protein of Escherichia coli maintains the negative supercoiling of DNA against thermal stress. J Biol Chem 271, 29407-29414.

Okanishi, H., Kim, K., Masui, R., and Kuramitsu, S. (2013). Acetylome with structural mapping reveals the significance of lysine acetylation in Thermus thermophilus. J Proteome Res 12, 3952-3968.

Ouidir, T., Kentache, T., and Hardouin, J. (2016). Protein lysine acetylation in bacteria: Current state of the art. Proteomics 16, 301-309.

Owen, D.J., Ornaghi, P., Yang, J.C., Lowe, N., Evans, P.R., Ballario, P., Neuhaus, D., Filetici, P., and Travers, A.A. (2000). The structural basis for the recognition of acetylated histone $\mathrm{H} 4$ by the bromodomain of histone acetyltransferase gen $5 \mathrm{p}$. The EMBO journal 19, 6141-6149.

Peng, S., Stephan, R., Hummerjohann, J., and Tasara, T. (2014). Evaluation of three reference genes of Escherichia coli for mRNA expression level normalization in view of salt and organic acid stress exposure in food. FEMS microbiology letters 355, 78-82.

Perry, K., and Mondragon, A. (2002). Biochemical characterization of an invariant histidine involved in Escherichia coli DNA topoisomerase I catalysis. J Biol Chem 277, 13237-13245.

Peter, B.J., Arsuaga, J., Breier, A.M., Khodursky, A.B., Brown, P.O., and Cozzarelli, N.R. (2004). Genomic transcriptional response to loss of chromosomal supercoiling in Escherichia coli. Genome Biol 5, R87.

Peter, B.J., Ullsperger, C., Hiasa, H., Marians, K.J., and Cozzarelli, N.R. (1998). The structure of supercoiled intermediates in DNA replication. Cell 94, 819-827.

Pommier, Y. (2006). Topoisomerase I inhibitors: camptothecins and beyond. Nat Rev Cancer 6, 789-802. 
Pommier, Y. (2013). Drugging topoisomerases: lessons and challenges. ACS Chem Biol $8,82-95$.

Postow, L., Crisona, N.J., Peter, B.J., Hardy, C.D., and Cozzarelli, N.R. (2001). Topological challenges to DNA replication: conformations at the fork. Proc Natl Acad Sci U S A 98, 8219-8226.

Pougovkina, O., te Brinke, H., Ofman, R., van Cruchten, A.G., Kulik, W., Wanders, R.J., Houten, S.M., and de Boer, V.C. (2014). Mitochondrial protein acetylation is driven by acetyl-CoA from fatty acid oxidation. Hum Mol Genet 23, 3513-3522.

Prabakaran, S., Lippens, G., Steen, H., and Gunawardena, J. (2012). Post-translational modification: nature's escape from genetic imprisonment and the basis for dynamic information encoding. Wiley Interdiscip Rev Syst Biol Med 4, 565-583.

Pruss, G.J., Manes, S.H., and Drlica, K. (1982). Escherichia coli DNA topoisomerase I mutants: increased supercoiling is corrected by mutations near gyrase genes. Cell 31,3542.

Qi, H., Menzel, R., and Tse-Dinh, Y.C. (1996). Effect of the deletion of the sigma 32dependent promoter ( $\mathrm{P} 1)$ of the Escherichia coli topoisomerase I gene on thermotolerance. Mol Microbiol 21, 703-711.

Qi, H., Menzel, R., and Tse-Dinh, Y.C. (1997). Regulation of Escherichia coli topA gene transcription: involvement of a sigmaS-dependent promoter. J Mol Biol 267, 481-489.

Ramakrishnan, R., Schuster, M., and Bourret, R.B. (1998). Acetylation at Lys-92 enhances signaling by the chemotaxis response regulator protein CheY. Proc Natl Acad Sci U S A 95, 4918-4923.

Richmond, C.S., Glasner, J.D., Mau, R., Jin, H., and Blattner, F.R. (1999). Genome-wide expression profiling in Escherichia coli K-12. Nucleic Acids Res 27, 3821-3835.

Roca, J. (2011a). The torsional state of DNA within the chromosome. Chromosoma 120, 323-334.

Roca, J. (2011b). Transcriptional inhibition by DNA torsional stress. Transcription 2, 8285 .

Scaglione, K.M., Basrur, V., Ashraf, N.S., Konen, J.R., Elenitoba-Johnson, K.S., Todi, S.V., and Paulson, H.L. (2013). The ubiquitin-conjugating enzyme (E2) Ube2w ubiquitinates the $\mathrm{N}$ terminus of substrates. J Biol Chem 288, 18784-18788.

Schilling, B., Christensen, D., Davis, R., Sahu, A.K., Hu, L.I., Walker-Peddakotla, A., Sorensen, D.J., Zemaitaitis, B., Gibson, B.W., and Wolfe, A.J. (2015). Protein acetylation dynamics in response to carbon overflow in Escherichia coli. Mol Microbiol 98, 847-863. 
Schoeffler, A.J., and Berger, J.M. (2008). DNA topoisomerases: harnessing and constraining energy to govern chromosome topology. Q Rev Biophys 41, 41-101.

Seki, M., Nakagawa, T., Seki, T., Kato, G., Tada, S., Takahashi, Y., Yoshimura, A., Kobayashi, T., Aoki, A., Otsuki, M., et al. (2006). Bloom helicase and DNA topoisomerase IIIalpha are involved in the dissolution of sister chromatids. Molecular and cellular biology 26, 6299-6307.

Shemorry, A., Hwang, C.S., and Varshavsky, A. (2013). Control of protein quality and stoichiometries by N-terminal acetylation and the N-end rule pathway. Mol Cell 50, 540551.

Singh, R., Ledesma, K.R., Chang, K.T., and Tam, V.H. (2010). Impact of recA on levofloxacin exposure-related resistance development. Antimicrobial agents and chemotherapy 54, 4262-4268.

Skourti-Stathaki, K., and Proudfoot, N.J. (2014). A double-edged sword: R loops as threats to genome integrity and powerful regulators of gene expression. Genes \& development 28, 1384-1396.

Sobetzko, P. (2016). Transcription-coupled DNA supercoiling dictates the chromosomal arrangement of bacterial genes. Nucleic Acids Res 44, 1514-1524.

Sokalingam, S., Raghunathan, G., Soundrarajan, N., and Lee, S.G. (2012). A study on the effect of surface lysine to arginine mutagenesis on protein stability and structure using green fluorescent protein. PloS one 7, e40410.

Song, L., Wang, G., Malhotra, A., Deutscher, M.P., and Liang, W. (2016). Reversible acetylation on Lys501 regulates the activity of RNase II. Nucleic Acids Res 44, 1979-1988.

Starai, V.J., Celic, I., Cole, R.N., Boeke, J.D., and Escalante-Semerena, J.C. (2002). Sir2dependent activation of acetyl-CoA synthetase by deacetylation of active lysine. Science 298, 2390-2392.

Starai, V.J., and Escalante-Semerena, J.C. (2004). Identification of the protein acetyltransferase (Pat) enzyme that acetylates acetyl-CoA synthetase in Salmonella enterica. J Mol Biol 340, 1005-1012.

Stewart, N., Feng, J., Liu, X., Chaudhuri, D., Foster, J.W., Drolet, M., and Tse-Dinh, Y.C. (2005). Loss of topoisomerase I function affects the RpoS-dependent and GAD systems of acid resistance in Escherichia coli. Microbiology 151, 2783-2791.

Stockum, A., Lloyd, R.G., and Rudolph, C.J. (2012). On the viability of Escherichia coli cells lacking DNA topoisomerase I. BMC Microbiol 12, 26. 
Stuckey, R., Garcia-Rodriguez, N., Aguilera, A., and Wellinger, R.E. (2015). Role for RNA:DNA hybrids in origin-independent replication priming in a eukaryotic system. Proc Natl Acad Sci U S A 112, 5779-5784.

Su, X., Wellen, K.E., and Rabinowitz, J.D. (2016). Metabolic control of methylation and acetylation. Curr Opin Chem Biol 30, 52-60.

Tan, K., Zhou, Q., Cheng, B., Zhang, Z., Joachimiak, A., and Tse-Dinh, Y.C. (2015). Structural basis for suppression of hypernegative DNA supercoiling by $E$. coli topoisomerase I. Nucleic Acids Res 43, 11031-11046.

Tang, Y., Zhao, W., Chen, Y., Zhao, Y., and Gu, W. (2008). Acetylation is indispensable for p53 activation. Cell 133, 612-626.

Tatham, M.H., Plechanovova, A., Jaffray, E.G., Salmen, H., and Hay, R.T. (2013). Ube2W conjugates ubiquitin to alpha-amino groups of protein N-termini. Biochem J 453, 137-145.

Terekhova, K., Marko, J.F., and Mondragon, A. (2013). Studies of bacterial topoisomerases I and III at the single-molecule level. Biochem Soc Trans 41, 571-575.

Tessarz, P., and Kouzarides, T. (2014). Histone core modifications regulating nucleosome structure and dynamics. Nat Rev Mol Cell Biol 15, 703-708.

Thao, S., and Escalante-Semerena, J.C. (2011a). Biochemical and thermodynamic analyses of Salmonella enterica Pat, a multidomain, multimeric N(epsilon)-lysine acetyltransferase involved in carbon and energy metabolism. MBio 2.

Thao, S., and Escalante-Semerena, J.C. (2011b). Control of protein function by reversible Nvarepsilon-lysine acetylation in bacteria. Curr Opin Microbiol 14, 200-204.

Tiwari, P.B., Chapagain, P.P., Banda, S., Darici, Y., Uren, A., and Tse-Dinh, Y.C. (2016). Characterization of molecular interactions between Escherichia coli RNA polymerase and topoisomerase I by molecular simulations. FEBS letters 590, 2844-2851.

Travers, A., and Muskhelishvili, G. (2015). DNA structure and function. The FEBS journal 282, 2279-2295.

Tse-Dinh, Y.C. (1985). Regulation of the Escherichia coli DNA topoisomerase I gene by DNA supercoiling. Nucleic Acids Res 13, 4751-4763.

Tse-Dinh, Y.C. (2000). Increased sensitivity to oxidative challenges associated with topA deletion in Escherichia coli. J Bacteriol 182, 829-832.

Tse-Dinh, Y.C. (2009). Bacterial topoisomerase I as a target for discovery of antibacterial compounds. Nucleic Acids Res 37, 731-737. 
Tu, S., Guo, S.J., Chen, C.S., Liu, C.X., Jiang, H.W., Ge, F., Deng, J.Y., Zhou, Y.M., Czajkowsky, D.M., Li, Y., et al. (2015). YcgC represents a new protein deacetylase family in prokaryotes. Elife 4.

Tuduri, S., Crabbe, L., Conti, C., Tourriere, H., Holtgreve-Grez, H., Jauch, A., Pantesco, V., De Vos, J., Thomas, A., Theillet, C., et al. (2009). Topoisomerase I suppresses genomic instability by preventing interference between replication and transcription. Nat Cell Biol $11,1315-1324$.

The UniProt Consortium (2017). Controlled vocabulary of posttranslational modifications (PTM).

Usongo, V., and Drolet, M. (2014). Roles of type 1A topoisomerases in genome maintenance in Escherichia coli. PLoS genetics 10, e1004543.

Valjavec-Gratian, M., Henderson, T.A., and Hill, T.M. (2005). Tus-mediated arrest of DNA replication in Escherichia coli is modulated by DNA supercoiling. Mol Microbiol $58,758-773$.

Van Damme, P., Evjenth, R., Foyn, H., Demeyer, K., De Bock, P.J., Lillehaug, J.R., Vandekerckhove, J., Arnesen, T., and Gevaert, K. (2011). Proteome-derived peptide libraries allow detailed analysis of the substrate specificities of N(alpha)-acetyltransferases and point to hNaa10p as the post-translational actin N(alpha)-acetyltransferase. Mol Cell Proteomics 10, M110 004580.

Varland, S., Osberg, C., and Arnesen, T. (2015). N-terminal modifications of cellular proteins: The enzymes involved, their substrate specificities and biological effects. Proteomics 15, 2385-2401.

Varshavsky, A. (2011). The N-end rule pathway and regulation by proteolysis. Protein Sci 20, 1298-1345.

Viard, T., and de la Tour, C.B. (2007). Type IA topoisomerases: a simple puzzle? Biochimie 89, 456-467.

Vologodskii, A. (2016). Disentangling DNA molecules. Phys Life Rev 18, 118-134.

Vos, S.M., Tretter, E.M., Schmidt, B.H., and Berger, J.M. (2011). All tangled up: how cells direct, manage and exploit topoisomerase function. Nat Rev Mol Cell Biol 12, 827-841.

Wagner, G.R., and Payne, R.M. (2013). Widespread and enzyme-independent Nepsilonacetylation and Nepsilon-succinylation of proteins in the chemical conditions of the mitochondrial matrix. J Biol Chem 288, 29036-29045.

Wang, J.C. (1971). Interaction between DNA and an Escherichia coli protein omega. J Mol Biol 55, 523-533. 
Wang, J.C. (2002). Cellular roles of DNA topoisomerases: a molecular perspective. Nat Rev Mol Cell Biol 3, 430-440.

Wang, Q., Zhang, Y., Yang, C., Xiong, H., Lin, Y., Yao, J., Li, H., Xie, L., Zhao, W., Yao, Y., et al. (2010). Acetylation of metabolic enzymes coordinates carbon source utilization and metabolic flux. Science 327, 1004-1007.

Wang, Z., and Harshey, R.M. (1994). Crucial role for DNA supercoiling in Mu transposition: a kinetic study. Proc Natl Acad Sci U S A 91, 699-703.

Watson, J.D., and Crick, F.H. (1953). Molecular structure of nucleic acids; a structure for deoxyribose nucleic acid. Nature 171, 737-738.

Weinert, B.T., Iesmantavicius, V., Wagner, S.A., Scholz, C., Gummesson, B., Beli, P., Nystrom, T., and Choudhary, C. (2013). Acetyl-phosphate is a critical determinant of lysine acetylation in E. coli. Mol cell 51, 265-272.

Weinert, B.T., Satpathy, S., Hansen, B.K., Lyon, D., Jensen, L.J., and Choudhary, C. (2017). Accurate quantification of site-specific acetylation stoichiometry reveals the impact of sirtuin deacetylase CobB on the E. coli acetylome. Mol Cell Proteomics 16, 759769.

Weinstein-Fischer, D., Elgrably-Weiss, M., and Altuvia, S. (2000). Escherichia coli response to hydrogen peroxide: a role for DNA supercoiling, topoisomerase I and Fis. Mol Microbiol 35, 1413-1420.

Wells, N.J., Addison, C.M., Fry, A.M., Ganapathi, R., and Hickson, I.D. (1994). Serine 1524 is a major site of phosphorylation on human topoisomerase II alpha protein in vivo and is a substrate for casein kinase II in vitro. J Biol Chem 269, 29746-29751.

Wolfe, A.J. (2005). The acetate switch. Microbiol Mol Biol Rev 69, 12-50.

Wolfe, A.J. (2016). Bacterial protein acetylation: new discoveries unanswered questions. Curr Genet 62, 335-341.

Wolfe, A.J., Chang, D.E., Walker, J.D., Seitz-Partridge, J.E., Vidaurri, M.D., Lange, C.F., Pruss, B.M., Henk, M.C., Larkin, J.C., and Conway, T. (2003). Evidence that acetyl phosphate functions as a global signal during biofilm development. Mol Microbiol 48, 977988.

Xie, L., Wang, X., Zeng, J., Zhou, M., Duan, X., Li, Q., Zhang, Z., Luo, H., Pang, L., Li, W., et al. (2015). Proteome-wide lysine acetylation profiling of the human pathogen Mycobacterium tuberculosis. Int J Biochem Cell Biol 59, 193-202.

Yan, J., Barak, R., Liarzi, O., Shainskaya, A., and Eisenbach, M. (2008). In vivo acetylation of CheY, a response regulator in chemotaxis of Escherichia coli. J Mol Biol 376, 12601271. 
Yang, J., Annamalai, T., Cheng, B., Banda, S., Tyagi, R., and Tse-Dinh, Y.C. (2015). Antimicrobial Susceptibility and SOS-Dependent Increase in Mutation Frequency Are Impacted by Escherichia coli Topoisomerase I C-Terminal Point Mutation. Antimicrob Agents Chemother 59, 6195-6202.

Yu, B.J., Kim, J.A., Moon, J.H., Ryu, S.E., and Pan, J.G. (2008). The diversity of lysineacetylated proteins in Escherichia coli. J Microbiol Biotechnol 18, 1529-1536.

Zechiedrich, E.L., Khodursky, A.B., Bachellier, S., Schneider, R., Chen, D., Lilley, D.M., and Cozzarelli, N.R. (2000). Roles of topoisomerases in maintaining steady-state DNA supercoiling in Escherichia coli. J Biol Chem 275, 8103-8113.

Zencheck, W.D., Xiao, H., and Weiss, L.M. (2012). Lysine post-translational modifications and the cytoskeleton. Essays Biochem 52, 135-145.

Zhang, J., Sprung, R., Pei, J., Tan, X., Kim, S., Zhu, H., Liu, C.F., Grishin, N.V., and Zhao, Y. (2009a). Lysine acetylation is a highly abundant and evolutionarily conserved modification in Escherichia coli. Mol Cell Proteomics 8, 215-225.

Zhang, K., Zheng, S., Yang, J.S., Chen, Y., and Cheng, Z. (2013a). Comprehensive profiling of protein lysine acetylation in Escherichia coli. J Proteome Res 12, 844-851.

Zhang, Q., Zhou, A., Li, S., Ni, J., Tao, J., Lu, J., Wan, B., Li, S., Zhang, J., Zhao, S., et al. (2016). Reversible lysine acetylation is involved in DNA replication initiation by regulating activities of initiator DnaA in Escherichia coli. Sci Rep 6, 30837.

Zhang, Q.F., Gu, J., Gong, P., Wang, X.D., Tu, S., Bi, L.J., Yu, Z.N., Zhang, Z.P., Cui, Z.Q., Wei, H.P., et al. (2013b). Reversibly acetylated lysine residues play important roles in the enzymatic activity of Escherichia coli N-hydroxyarylamine O-acetyltransferase. The FEBS journal 280, 1966-1979.

Zhang, X., Yuan, Z., Zhang, Y., Yong, S., Salas-Burgos, A., Koomen, J., Olashaw, N., Parsons, J.T., Yang, X.J., Dent, S.R., et al. (2007). HDAC6 modulates cell motility by altering the acetylation level of cortactin. Mol cell 27, 197-213.

Zhang, Y., Zhang, M., Dong, H., Yong, S., Li, X., Olashaw, N., Kruk, P.A., Cheng, J.Q., Bai, W., Chen, J., et al. (2009b). Deacetylation of cortactin by SIRT1 promotes cell migration. Oncogene 28, 445-460.

Zhang, Z., Cheng, B., and Tse-Dinh, Y.C. (2011). Crystal structure of a covalent intermediate in DNA cleavage and rejoining by Escherichia coli DNA topoisomerase I. Proc Natl Acad Sci U S A 108, 6939-6944.

Zhao, K., Chai, X., and Marmorstein, R. (2004). Structure and substrate binding properties of cobB, a Sir2 homolog protein deacetylase from Escherichia coli. J Mol Biol 337, 731741. 
Zhou, K., Zhou, L., Lim, Q., Zou, R., Stephanopoulos, G., and Too, H.P. (2011). Novel reference genes for quantifying transcriptional responses of Escherichia coli to protein overexpression by quantitative PCR. BMC Mol Biol 12, 18.

Zhou, Q., Zhou, Y.N., Jin, D.J., and Tse-Dinh, Y.C. (2017). Deacetylation of topoisomerase I is an important physiological function of E. coli CobB. Nucleic Acids Res $45,5349-5358$

Zhu, C.X., Samuel, M., Pound, A., Ahumada, A., and Tse-Dinh, Y.C. (1995). Expression and DNA-binding properties of the $14 \mathrm{~K}$ carboxyl terminal fragment of Escherichia coli DNA topoisomerase I. Biochem Mol Biol Int 35, 375-385.

Zhu, C.X., and Tse-Dinh, Y.C. (1994). Binding of Zn(II) to Escherichia coli DNA topoisomerase I. Biochem Mol Biol Int 33, 195-204.

Zhu, C.X., and Tse-Dinh, Y.C. (2000). The acidic triad conserved in type IA DNA topoisomerases is required for binding of $\mathrm{Mg}(\mathrm{II})$ and subsequent conformational change. $\mathrm{J}$ Biol Chem 275, 5318-5322. 
VITA

QINGXUAN ZHOU

110 SW $109^{\text {th }}$ AVE APT8, Miami, FL 33174

$\begin{array}{ll}2008-2012 & \begin{array}{l}\text { B.S., Pharmacy } \\ \text { China Pharmaceutical University } \\ \text { Nanjing, China }\end{array} \\ & \text { Teaching Assistant } \\ 2012-2013 & \begin{array}{l}\text { Florida International University } \\ \text { Miami, FL }\end{array} \\ & \text { Research Assistant } \\ 2017-2016 & \text { Florida International University } \\ & \text { Miami, FL }\end{array}$

\section{PUBLICATIONS AND PRESENTATIONS}

Zhou, Q., Zhou, Y. N., Jin, D. J., \& Tse-Dinh, Y. C. (2017). Deacetylation of topoisomerase I is an important physiological function of E. coli CobB. Nucleic Acids Res 45, 5349-5358

Cheng,B., Zhou,Q., Weng,L., Leszyk,J.D., Greenberg,M.M., and Tse-Dinh,Y.C. (2016) Identification of proximal sites for unwound DNA substrate in E. coli topoisomerase I with oxidative crosslinking. FEBS Lett., 591(1):28-38.

Tan,K., Zhou,Q., Cheng,B., Zhang,Z., Joachimiak,A. and Tse-Dinh,Y.C. (2015) Structural basis for suppression of hypernegative DNA supercoiling by E. coli topoisomerase I. Nucleic Acids Res., 43, 11031-11046.

Zhou,Q., and Tse-Dinh, Y.C. Deacetylation of Topoisomerase I is an important physiological function of E. coli CobB. Gordon Research Conference-DNA

Topoisomerases, August 7-12,2016, Newry, ME

Zhou,Q., and Tse-Dinh, Y.C. The function of lysine acetylation on E. coli Topoisomerase I. Florida Branch of American Society for Microbiology, October 9-11, 2015, Cocoa Beach, FL

Zhou,Q., and Tse-Dinh, Y.C. Acetylation Modification on E. coli Topoisomerase I. FIU Graduate Student Appreciation Week, April 6-7, 2015, Miami, FL 
Zhou,Q., and Tse-Dinh, Y.C. Lysine acetylation on E. coli Topoisomerase I. 17th Annual Biomedical and Comparative Immunology Symposium, March 26-27, 2014, Miami, FL

Zhou,Q., and Tse-Dinh, Y.C. Lysine acetylation of E. coli Topoisomerase I. Gordon Research Conference-DNA Topoisomerases, August 10-15, 2014, Newry, ME

Zhou,Q., and Tse-Dinh, Y.C. Acetylation Modification of E. coli Topoisomerase I. FIU Graduate Student Appreciation Week, March 31-April 4, 2014, Miami, FL

Zhou,Q., and Tse-Dinh, Y.C. Acetylation Modification of E. coli Topoisomerase I. FIU Chemistry Department Annual Visitation Day, March 7, 2014, Miami, FL 